NBER WORKING PAPER SERIES

\title{
CONTROLLING FOR THE COMPROMISE EFFECT DEBIASES ESTIMATES OF RISK PREFERENCE PARAMETERS
}

Jonathan P. Beauchamp

Daniel J. Benjamin

Christopher F. Chabris

David I. Laibson

Working Paper 21792

http://www.nber.org/papers/w21792

\author{
NATIONAL BUREAU OF ECONOMIC RESEARCH \\ 1050 Massachusetts Avenue \\ Cambridge, MA 02138 \\ December 2015
}

We thank Helga Fehr-Duda, Philipp Koellinger, Kevin McCabe, Ted OíDonoghue, Matthew Rabin, Charlie Sprenger, as well as the Editor Charles Noussair and two anonymous referees, for helpful comments. For research assistance, we are grateful to Jonathan Cohen, Brice Cooke, Jaesun Lee, and especially Brendan Price, Alexandra Roulet, and Stephen Tino. Research reported in this publication was supported by the National Institute on Aging of the National Institutes of Health under Award Numbers R01AG021650 and P01AG005842 to the NBER and R21AG037741 to Cornell University. The content is the sole responsibility of the authors and does not necessarily represent the official views of the National Institutes of Health or of the National Bureau of Economic Research. Beauchamp and Laibson thank the Pershing Square Fund for Research on the Foundations of Human Behavior for financial support.

NBER working papers are circulated for discussion and comment purposes. They have not been peerreviewed or been subject to the review by the NBER Board of Directors that accompanies official NBER publications.

(C) 2015 by Jonathan P. Beauchamp, Daniel J. Benjamin, Christopher F. Chabris, and David I. Laibson. All rights reserved. Short sections of text, not to exceed two paragraphs, may be quoted without explicit permission provided that full credit, including $\odot$ notice, is given to the source. 
Controlling for the Compromise Effect Debiases Estimates of Risk Preference Parameters

Jonathan P. Beauchamp, Daniel J. Benjamin, Christopher F. Chabris, and David I. Laibson NBER Working Paper No. 21792

December 2015, Revised November 2018

JEL No. B49,D03,D14,D83,G11

\begin{abstract}
$\underline{\text { ABSTRACT }}$
The compromise effect arises when options near the "middle" of a choice set are more appealing. The compromise effect poses conceptual and practical problems for economic research: by influencing choices, it distorts revealed preferences, biasing researchers' inferences about deep (i.e., domain general) preferences. We propose and estimate an econometric model that disentangles and identifies both deep preferences and the context-dependent compromise effect. We demonstrate our method using data from an experiment with 550 participants who made choices over lotteries from multiple price lists. Following prior work, we manipulate the compromise effect by varying the middle options of each multiple price list and then estimate risk preferences without modelling the compromise effect. These naïve parameter estimates are not robust: they change as the compromise effect is manipulated. To eliminate this bias, we incorporate the compromise effect directly into our econometric model. We show that this method generates robust estimates of risk preference parameters that are no longer sensitive to compromise-effect manipulations. This method can be applied to other settings that exhibit the compromise effect.
\end{abstract}

Jonathan P. Beauchamp

Department of Economics

Harvard University

Cambridge, MA 02138

beauchamp.jonathan@gmail.com

Daniel J. Benjamin

Center for Economics and Social Research

University of Southern California

635 Downie Way, Suite 312

Los Angeles, CA 90089-3332

and NBER

daniel.benjamin@gmail.com
Christopher F. Chabris

100 North Academy Avenue

Danville, Penn 17822-3069

United States

chabris@gmail.com

David I. Laibson

Department of Economics

Littauer M-12

Harvard University

Cambridge, MA 02138

and NBER

dlaibson@gmail.com 


\section{Introduction}

The compromise effect arises when options in a choice set can be ordered on common dimensions

or attributes (such as price, quantity, size, or intensity), and decision makers tend to select the options in the "middle" of the choice set. For example, suppose a group of respondents were asked whether they wanted a free nature hike of either 1 mile or 4 miles. Now suppose that a different, otherwise identical group were asked whether they preferred a free nature hike of 1, 4, or 7 miles. A compromise effect could lead to a greater fraction of respondents choosing 4 miles in the second choice set (see Simonson 1989 for a closely related empirical result and Kamenica 2008 for a discussion of microfoundations).

The compromise effect poses conceptual and practical problems for economic research. By influencing choices, the compromise effect distorts revealed preferences, biasing researchers' inferences about deep (i.e., domain general) preferences.

In this paper, we propose and estimate an econometric model that disentangles and separately identifies both the deep preferences and the (situational) compromise effect that is influencing the expression of those deep preferences. To demonstrate our approach, we conduct a laboratory experiment with 550 participants in which we elicit risk preferences using a multiple price list (MPL). We study this context because, despite the limitations of the MPL procedure, it is among the most commonly used methods to elicit preferences in the economics literature (e.g., Tversky and Kahneman 1992, Holt and Laury 2002, Harrison, List, and Towe 2007, Andersen, Harrison, Lau, and Rutström 2008) and because the compromise effect has been carefully and robustly documented already in the context of inferring risk preferences using an MPL (Birnbaum 1992, Harrison, Lau, Rutström, and Sullivan 2005, Andersen, Harrison, Lau, and Rutström 2006, Harrison, Lau, and Rutström 2007). We use the term compromise effect as short-hand for a bias toward the middle option, which is what these papers document.

The screenshot below is drawn from our own experiment and is typical of MPL experiments. In this example, a participant is asked to make seven binary choices. Each of the seven choices is between a gamble and a sure-thing alternative. The gamble doesn't change across the seven rows, while the sure-thing alternative varies from high to low. 
A gamble gives you a $10 \%$ chance of gaining $\$ 100$ and a $90 \%$ chance of gaining $\$ 50$ instead.

Would you rather...

$\begin{array}{lllll}\text { (a) } & \text { Take the gamble } & \text { OR } & \text { Gain } \$ 57.00 \\ \text { (b) } & \text { Take the gamble } & \text { OR } & \text { Gain } \$ 56.90 \\ \text { (c) } & \text { Take the gamble } & \text { OR } & \text { Gain } \$ 56.70 \\ \text { (d) } & \text { Take the gamble } & \text { OR } & \text { Gain } \$ 56.40 \\ \text { (e) } & \text { Take the gamble } & \text { OR } & \text { Gain } \$ 55.90 \\ \text { (f) } & \text { Take the gamble } & \text { OR } & \text { Gain } \$ 55.00 \\ \text { (g) } & \text { Take the gamble } & \text { OR } & \text { Gain } \$ 53.60\end{array}$

A subject who displayed a strong compromise effect would act as if she were indifferent between the gamble and the sure-thing in the middle row, which is row (d). Such indifference would imply that she is risk seeking because the gamble has a lower expected value than the sure thing in row (d). In this example, a strong compromise effect would lead a participant who may otherwise be risk-averse to make risk-seeking choices.

Following prior work (Birnbaum 1992, Harrison, Lau, Rutström, and Sullivan 2005, Andersen, Harrison, Lau, and Rutström 2006, Harrison, Lau, and Rutström 2007, and Harrison, List, and Towe 2007), we experimentally vary the middle option using scale manipulations. Specifically, we hold the lowest and highest alternatives of the MPL fixed and manipulate the locations of the five intermediate outcomes within the scale. For example, compare the screenshot above to the screenshot that follows, which has new alternatives in rows (b) through (f), although rows (a) and (g) are the same. With respect to this second MPL, an agent who acts as if the middle option, row (d), is her indifference point would be judged to be risk averse.

A gamble gives you a $10 \%$ chance of gaining $\$ 100$ and a $90 \%$
Would you rather...
$\begin{array}{lllll}\text { (a) } & \text { Take the gamble } & \text { OR } & \text { Gain } \$ 57.00 \\ \text { (b) } & \text { Take the gamble } & \text { OR } & \text { Gain } \$ 55.60 \\ \text { (c) } & \text { Take the gamble } & \text { OR } & \text { Gain } \$ 54.70 \\ \text { (d) } & \text { Take the gamble } & \text { OR } & \text { Gain } \$ 54.20 \\ \text { (e) } & \text { Take the gamble } & \text { OR } & \text { Gain } \$ 53.90 \\ \text { (f) } & \text { Take the gamble } & \text { OR } & \text { Gain } \$ 53.70 \\ \text { (g) } & \text { Take the gamble } & \text { OR } & \text { Gain } \$ 53.60\end{array}$

In our experiment, each participant is exposed to one of five different scale treatment conditions.

To econometrically disentangle risk preferences from the compromise effect, we augment a 
discrete-choice model with additional parameters that represent a penalty for choosing a switch point further from the middle. Note that our approach of incorporating the compromise effect into the econometric model is different from including treatment-condition indicators as controls. Simply controlling for treatment condition would not identify domain-general preferences because the compromise effect can influence choices in every treatment condition (i.e., there is no benchmark, compromise-effect-free treatment condition).

The deep preferences we study in the current paper are prospect-theoretic preferences over risky lotteries (e.g., Tversky and Kahneman 1992, Wakker 2010, Bruhin, Fehr-Duda, and Epper 2010). Our ex-ante hypotheses focus on two parameters: curvature $\gamma$ (which captures risk aversion over gains and risk seeking over losses) and loss aversion $\lambda$ (which captures the degree to which people dislike losses more than they like gains). ${ }^{1}$ Our analysis yields three main findings.

First, our estimates of the compromise-effect parameters replicate the findings from earlier work that participants have a bias toward choosing a switch point in the middle rows of the MPL (e.g., Harrison, Lau, Rutström, and Sullivan 2005; see other references above). Moreover, our quantitative estimates indicate that the bias is sizeable; we estimate that the attractiveness of the middle rows relative to the extreme rows represents $17 \%-23 \%$ of the prospects' monetary value.

Second, when we estimate the prospect-theory model without controls for the compromise effect, the scale manipulations have a very powerful effect on the (mis-) estimated preference parameters. In particular, the compromise effect is strong enough to cause us to estimate either risk seeking (as predicted by prospect theory) or risk aversion (the opposite of what is predicted by prospect theory) in the loss domain, depending on the scale manipulations. The compromise effect is also strong enough that, when manipulated, it can make behavior look as if there is essentially no loss aversion (see the results for the Pull 2 treatment below).

Third, when we estimate the prospect-theory parameters while including additional parameters to capture the compromise effect, our estimates of $\gamma$ and $\lambda$ are robust across the five scale treatment conditions. The robustness of these preference-parameter estimates implies that they are not biased

\footnotetext{
${ }^{1}$ We predicted that our scaling manipulations would not substantially change the estimated parameters of the probability weighting function, because the prospects all have "probability-flipped" variants: i.e., for each MPL featuring a prospect with probability $p$ of monetary outcome $x_{H}$ and probability $1-p$ of monetary outcome $x_{L}$, the experiment includes another MPL featuring a probability-flipped prospect with probability $1-p$ of outcome $x_{H}$ and probability $p$ of outcome $x_{L}$. Scaling manipulations will have (approximately) offsetting effects with respect to the probability weighting function for these two probability-flipped prospects.
} 
by the compromise effect. (When estimating the model pooling all of our experimental data, our estimates are $\hat{\gamma}=0.24$ and $\hat{\lambda}=1.31$, which fall within the range of estimates in the existing literature, albeit with $\hat{\lambda}$ toward the lower end of the range.)

In addition to the scale manipulations described above, we also study the effect of telling experimental participants the expected value of the risky prospects. We hypothesized that this manipulation would anchor the participants on the expected value, thereby nudging their preferences toward risk neutrality. However, we find that expected value information does not affect measured risk aversion nor measured loss aversion. Our null effect echoes the findings of Lichtenstein, Slovic, and Zink (1969) and Montgomery and Adelbratt (1982). However, Harrison and Rutström (2008) do find that providing expected value information significantly decreases risk aversion. The difference may arise because the prospects in their experiment are relatively complex, each involving four possible outcomes (versus one or two in our experiment).

This paper contributes to the literature on the compromise effect by estimating a model that explicitly accounts for the compromise effect and enables us to separately estimate it from risk preferences. Our sample is substantially larger than those used in earlier work, which allows us to precisely estimate the effects of the scale manipulations. Moreover, because we pose gambles involving losses as well as gambles involving gains, we can study the effect of scale manipulations not only on risk aversion over gains, but also on risk aversion over losses and on loss aversion. In addition, we provide estimates of the economic magnitude and importance of the compromise effect relative to the prospects' monetary value, and we examine the demographic correlates of the parameters in our econometric model. A limitation of our experiment is that only one out of its four parts (which involves 28 of the 62 sets of choices we analyze) is incentivized. Reassuringly, all of our results still hold when we restrict attention to the incentivized data.

The rest of the paper is organized as follows. In Section 2, we discuss our experimental design. In Section 3, we describe our econometric discrete-choice model, which incorporates the compromise effect. In Section 4, we list and discuss the five formal hypotheses that we test. In Section 5, we report the results of the estimation of our model, and we test the robustness of the estimates to the scale manipulations. Section 6 parallels Section 5 but examines the prospect-theory model without controls for the compromise effect. Section 7 estimates the economic magnitude and importance of the compromise effect in our data. Sections 8 briefly analyzes the demographic correlates of 
the main parameters of our econometric model (including $\gamma, \lambda$, and parameters that capture the compromise effect). Section 9 briefly discusses the results of our expected value manipulation. Section 10 concludes.

\section{Experiment}

\subsection{Design}

Throughout the experiment, we employ the Multiple Price List (MPL) elicitation method (Tversky and Kahneman 1992, Holt and Laury 2002). At the top of each computer screen, a fixed prospect is presented. The fixed prospect is usually a non-degenerate lottery; it is "fixed" in the sense that it is an option in all of the binary choices on that screen. (The fixed prospect changes across screens.) On each screen, seven binary choices are listed below the fixed prospect. Each binary choice is made between the fixed prospect (at the top of the screen) and what we refer to as an alternative (or alternative prospect). The alternatives vary within a screen, with one alternative for each of the seven binary choices. In some (but not all) cases, the alternatives are sure things. Screenshots of the experiment are shown in the Introduction as well as in the Appendix, and the original instructions of the experiment are shown in the Online Appendix.

Our set-up for eliciting risk preferences is standard. Indeed, we designed many details of our experiment - such as giving participants choices between a fixed prospect and seven alternativesto closely follow Tversky and Kahneman's (1992; henceforth T\&K) experiment in their paper that introduced Cumulative Prospect Theory (CPT). Moreover, our set of fixed prospects is identical to the set used by T\&K. Further mimicking T\&K's procedure, our computer program enforces consistency in the participants' choices by requiring participants to respond monotonically to the seven choices on the screen. ${ }^{2}$ Our algorithm for generating the seven alternatives is explained in Section 2.2 and in the Online Appendix, where we also list the complete set of fixed prospects and alternatives. ${ }^{3}$

\footnotetext{
${ }^{2}$ More precisely, participants have to select only two circles: the one corresponding to the worst alternative outcome they prefer to the fixed prospect and the one corresponding to the fixed prospect in the following row. The other circles are auto-filled. This procedure is a version of the "Switching MPL" (or "sMPL", see Andersen et al. 2006). This procedure reduces participants' fatigue and produces clean data, but it might have the unintended effect of biasing participants to select a row near the middle and thus exaggerating the compromise effect.

${ }^{3}$ Our procedure differs from T\&K's in three ways. First, T\&K do not report the actual values they used. Second, while their gambles were all hypothetical, our "Part A" gambles are incentivized. Third, for each screen, T\&K
} 
Each participant faces a total of 64 screens in the experiment, each of which contains seven choices between a fixed prospect and alternatives. There are four types of screens that differ from each other in the kinds of prospects and alternatives they present. To make it easier for participants to correctly understand the choices we are presenting to them, we divide the experiment into four sequential parts (each with its own instruction screen), with each part containing a single type of fixed prospect and a single type of alternative. The order of the screens is randomized within each part, with half the participants completing the screens in one order, and the other half completing the screens in the reverse order.

In Part A, the fixed prospects are in the gain domain, and the alternatives are sure gains (as in the example screens in the Introduction). There are 28 fixed prospects that differ both in probabilities and money amounts, which range from $\$ 0$ to $\$ 400$. The seven alternatives for each fixed prospect range from the fixed prospect's certainty equivalent for a CRRA expected-utilitymaximizer with CRRA parameter $\gamma=0.99$ to the certainty equivalent for $\gamma=-1$ (which is risk seeking). ${ }^{4}$ Because the range of estimates of $\gamma$ in the literature falls well within this interval (Booij, van Praag, and van de Kuilen, 2010), the interval likely covers the relevant range of alternatives for the participants. Each participant is told that there is a $1 / 6$ chance that one of his or her choices in Part A will be randomly selected and implemented for real stakes at the end of the experiment. The expected payout for a risk-neutral participant who rolls a 6 is about $\$ 100$. The remaining parts of the experiments involve hypothetical stakes.

In Part B, the fixed prospects now have outcomes in the loss domain, and the alternatives are sure losses. The 28 prospects and alternatives in Part B are identical to those in Part A but with all dollar amounts multiplied by -1 .

Parts $\mathrm{C}$ and $\mathrm{D}$ depart somewhat from the baseline format of our experiment, in that the alternatives are now risky prospects rather than sure things. Moreover, in Part C, the fixed prospect is the degenerate prospect of a sure thing of $\$ 0$ and is not listed at the top of each screen. The seven alternatives on each of the four screens in Part $\mathrm{C}$ are mixed prospects that have a $50 \%$ chance

implement a two-step procedure: after finding the point at which participants switch from preferring the alternative outcomes to preferring the fixed prospect, the participant make choices between the fixed prospect and a second set of seven alternative outcomes, linearly spaced between a value $25 \%$ higher than the lowest amount accepted in the first set and a value $25 \%$ lower than the highest amount rejected. We avoid this two-step procedure (which Harrison, Lau and Rutström, 2007, call an "Iterative Multiple Price List") to maintain incentive compatibility.

${ }^{4}$ We use $\gamma=0.99$ because $\gamma=1$ corresponds to log utility and implies a certainty equivalent of $\$ 0$ for any prospect with a chance of a $\$ 0$ outcome. 
of a loss and $50 \%$ chance of a gain. For example, one of the screens in Part $\mathrm{C}$ is:

A gamble gives you a $50 \%$ chance of losing $\$ 150$ and ...

(a) $\ldots$ a $50 \%$ chance of gaining $\$ 0.00$ instead.

(b) $\ldots$ a $50 \%$ chance of gaining $\$ 14.90$ instead.

(c) $\ldots$ a $50 \%$ chance of gaining $\$ 39.60$ instead.

(d) $\ldots$ a $50 \%$ chance of gaining $\$ 80.60$ instead.

(e) $\ldots$ a $50 \%$ chance of gaining $\$ 148.80$ instead.

(f) $\ldots$ a $50 \%$ chance of gaining $\$ 262.00$ instead.

(g) $\ldots$ a $50 \%$ chance of gaining $\$ 450.00$ instead.
Take the gamble

Take the gamble

Take the gamble

Take the gamble

Take the gamble

Take the gamble

Take the gamble
OR

OR

OR

OR

OR

OR

OR
Don't take the gamble

Don't take the gamble

Don't take the gamble

Don't take the gamble

Don't take the gamble

Don't take the gamble

Don't take the gamble

On any given screen, the amount of the possible loss is fixed, and the seven mixed prospects involve different amounts of the possible gain. Part C has four screens, each with a different loss amount: $\$ 25, \$ 50, \$ 100$, and $\$ 150$.

Part D also comprises four screens, each containing choices between a fixed $50 \%-50 \%$ risky prospect and seven alternative $50 \%-50 \%$ risky prospects. On two of the four screens, both the fixed prospect and the alternatives are mixed prospects, i.e., one possible outcome is a gain and the other is a loss, as in the following:

Gamble 1 gives you a $50 \%$ chance of losing $\$ 50$ and a $50 \%$ chance of gaining $\$ 150$

Gamble 2 gives you a $50 \%$ chance of losing $\$ 125$ and ...
(a) $\quad \ldots$ a $50 \%$ chance of gaining $\$ 375.00$ instead.
(b) $\quad \ldots$ a $50 \%$ chance of gaining $\$ 356.30$ instead.
C Take gamble 1 OR
Take gamble 2
(c) $\ldots$ a $50 \%$ chance of gaining $\$ 332.50$ instead.
Take gamble 1 OR
Take gamble 2
(d) $\ldots$ a $50 \%$ chance of gaining $\$ 302.00$ instead.
Take gamble 1 OR
Take gamble 2
(e) $\ldots$ a $50 \%$ chance of gaining $\$ 263.10$ instead.
Take gamble 1 OR
Take gamble 1 OR
Take gamble 2
(f) $\quad \ldots$ a $50 \%$ chance of gaining $\$ 213.40$ instead.
Take gamble 1 OR
Take gamble 2
(g) $\ldots$ a $50 \%$ chance of gaining $\$ 150.00$ instead.
Take gamble 1 OR
Take gamble 2
Take gamble 2

On the other two screens, the fixed and the alternative prospects involve only gains. On any given screen, one of the two possible realizations of the alternative prospect is fixed, and the seven choices on the screen involve different amounts of the other possible realization of that prospect. For each screen in Parts $\mathrm{C}$ and $\mathrm{D}$, the alternative prospects range from the amount that would make an individual with linear utility, no probability distortion, and loss insensitivity $(\lambda=0)$ indifferent to the fixed prospect to the amount that would make an individual with loss aversion $\lambda=3$ indifferent.

After Parts A-D, participants complete a brief questionnaire that asks age, race, educational 
background, standardized test scores, ZIP code of permanent residence, and parents' income (if the participant is a student) or own income (if not a student). It also asks a few self-reported behavioral questions, including general willingness to take risks and frequency of gambling.

\subsection{Treatments}

As detailed below, the experiment has a $5 \times 2$ design, with five "Pull" treatments, which vary the set of alternatives, crossed with two "EV" treatments, which vary whether the expected value of the prospects is displayed or not. Each participant is randomly assigned to one of the ten treatment cells and remains in this cell for all screens and all parts (A-D) of the experiment.

The Pull treatments allow us to assess whether the compromise effect impacts measured risk and loss preferences. The five treatments are identical in the set of fixed prospects and in the first and seventh alternative on each screen but differ from each other in the intermediate (the second through sixth) alternatives. For instance, in Part A for the illustrative fixed prospect above in the screenshots in the Introduction - a 10\% chance of gaining $\$ 100$ and a $90 \%$ chance of gaining $\$ 50$ - the alternatives (a) through (g) are shown in the positive half of Figure 1 for all five Pull treatments.

The five treatments are labeled Pull -2, Pull -1, Pull 0, Pull 1, and Pull 2. In the Pull 0 treatment, the alternatives are evenly spaced, aside from rounding to the nearest $\$ 0.10$, from the low amount of $\$ 53.60$ to the high amount of $\$ 57.00$. In the Pull 1 and the Pull 2 treatments, the intermediate alternatives are more densely concentrated at the monetary amounts closer to zero. These treatments are designed to resemble T\&K's experiment, in which the second through sixth alternatives are "logarithmically spaced between the extreme outcomes of the prospect" (T\&K, p. 305). Conversely, in the Pull -1 and Pull -2 treatments, the intermediate alternatives are more densely concentrated at the monetary amounts farther from zero. Pull 2 and Pull -2 are more skewed than Pull 1 and Pull -1. We refer to the different treatments as "Pulls" to convey the intuition that they pull the distributions of the intermediate alternatives toward zero (for the positive Pulls) or away from zero (for the negative Pulls). 

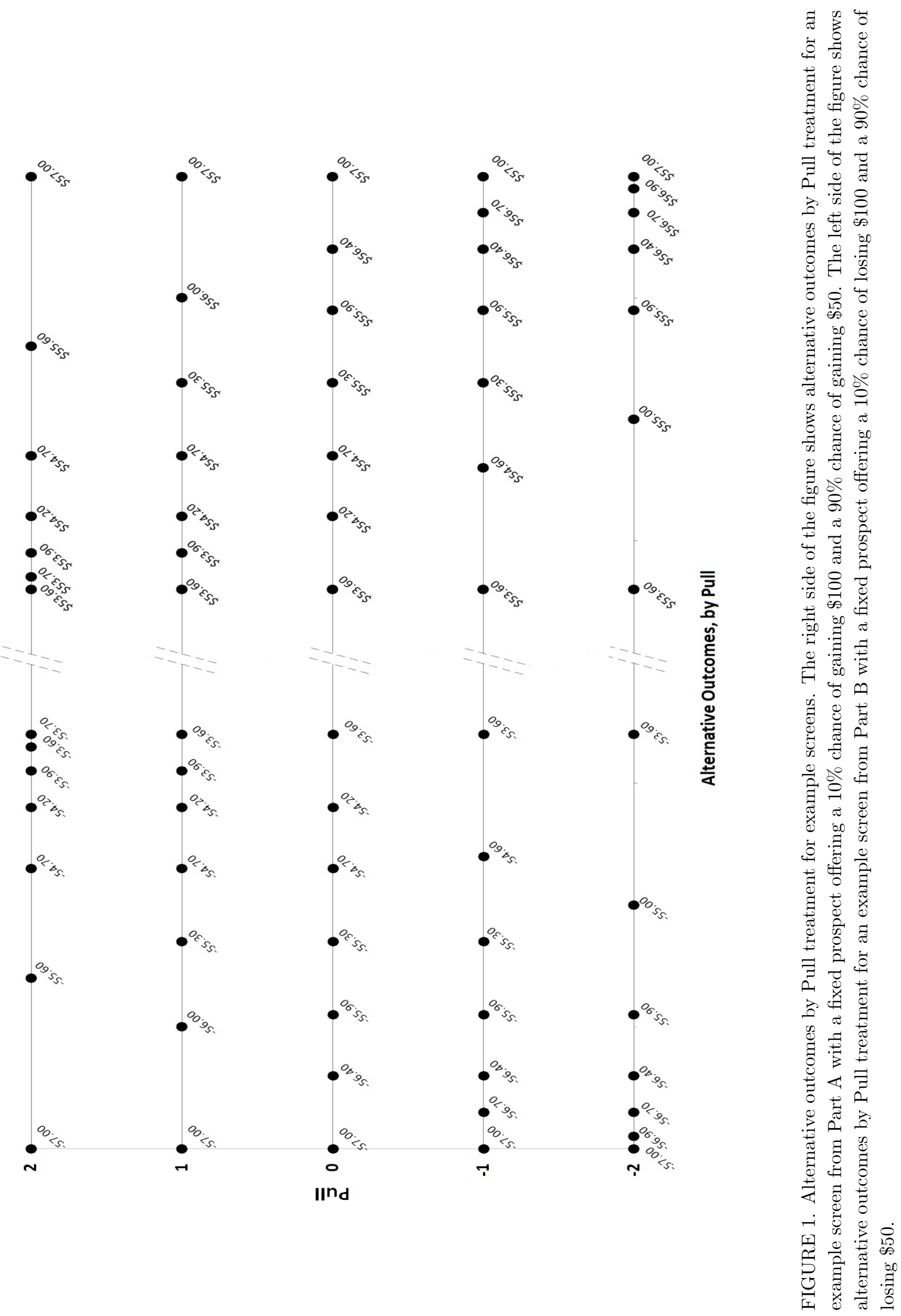
Analogously, in Parts C and D, Pull 1 and Pull 2 pull the distribution of the varying amounts of the intermediate alternative prospect on each screen toward zero, and Pull -1 and Pull -2 do the opposite. The Online Appendix describes the precise algorithm we use to determine the second through sixth alternatives and shows the complete set of fixed prospects and alternatives for each Pull treatment and for each part of the experiment.

The EV treatments differ in whether or not we inform participants about the expected values of the prospects. Because we anticipated that many participants would be unfamiliar with the concept of expected value, simple language is used in the "EV treatment" to describe it. For instance, in Part A, the following appears below the fixed prospect at the top of the screen: "On average, you would gain $\$ 55$ from taking this gamble."

\subsection{Procedures and Sample}

The experiment was run online from March 11 to March 20, 2010. Our sample was drawn from the Harvard Business School Computer Lab for Experimental Research's (CLER) online subject pool database. This database contains several thousand participants nationwide who are available to participate in online studies. Participants had to be at least 18 years old, eligible to receive payment in the U.S., and not on Harvard University's regular payroll. At the time we ran the experiment, members of the CLER online subject pool database were mainly recruited through flyer postings around neighboring campuses.

At the launch of the experiment, the CLER lab posted a description to advertise the experiment to the members of the online subject pool database. Any member of the pool could then participate until a sample size of 550 was reached. Each participant was pseudo-randomly assigned to one Pull and to one EV treatment to ensure that our treatments were well-balanced. A total of 521 participants completed all four parts of the experiment. The mean response time for the participants who completed the experiment in less than one hour was 32 minutes. ${ }^{5}$

In addition to the above-described incentive payment for Part A, participants were paid a total of $\$ 5$ if they began the experiment; $\$ 7$ if they completed Part A; $\$ 9$ if they completed Parts A and B; $\$ 11$ if they completed Parts A, B, and C; and $\$ 15$ if they completed all four parts of the

\footnotetext{
${ }^{5}$ Participants were allowed to complete the experiment in more than one session and some response times exceeded 24 hours. Of the 497 participants for whom we have response time data, 405 took less than an hour.
} 
experiment.

\subsection{Summary Statistics of the Raw Data from the Experiment}

Online Appendix Section 3 includes figures that show the percentage of choices where the safe option was chosen, by Pull and EV treatments, separately for Parts A, B, C, and D of the experiment. These figures give a first impression of the data we collected in our experiment, but caution is warranted in interpreting them because the different Pull treatments involve different sets of choices, and the raw data are thus not directly comparable across treatments.

\section{Model and Estimation}

\subsection{Baseline CPT Model}

We assume that participants' deep preferences can be modeled according to CPT. For prospect $P=\left(x_{H}, p_{H} ; x_{L}, p_{L}\right)$ with probability $p_{H}$ of monetary outcome $x_{H}$ and probability $p_{L}=1-p_{H}$ of monetary outcome $x_{L}$, we assume that utility has the form:

$$
U(P)=\left\{\begin{array}{ll}
\omega\left(p_{H}\right) \cdot u\left(x_{H}\right)+\left(1-\omega\left(p_{H}\right)\right) \cdot u\left(x_{L}\right) & \text { if } 0<x_{L}<x_{H} \\
-\omega\left(p_{L}\right) \cdot \lambda \cdot u\left(-x_{L}\right)-\left(1-\omega\left(p_{L}\right)\right) \cdot \lambda \cdot u\left(-x_{H}\right) & \text { if } x_{L}<x_{H}<0 \\
\omega\left(p_{H}\right) \cdot u\left(x_{H}\right)-\omega\left(p_{L}\right) \cdot \lambda \cdot u\left(-x_{L}\right) & \text { if } x_{L}<0<x_{H}
\end{array}\right\}
$$

where $\omega(\cdot)$ is the cumulative probability weighting function and satisfies $\omega(0)=0$ and $\omega(1)=1$, $u(\cdot)$ is the Bernoulli utility function and satisfies $u(0)=0$, and $\lambda$ is the coefficient of loss aversion. We assume that $u(\cdot)$ takes the CRRA (a.k.a. "power utility") form, $u(x)=\frac{x^{1-\gamma}}{1-\gamma}$, as is standard in the literature on CPT (e.g., Fox and Poldrack 2014; T\&K).

We use the Prelec (1998) probability weighting function:

$$
\omega(p)=\exp \left(-\beta(-\log (p))^{\alpha}\right)
$$

where $\alpha, \beta>0$. The $\alpha$ and $\beta$ parameters regulate the curvature and the elevation of $\omega(p)$, respectively. 


\subsection{Modeling the Compromise Effect}

We model the compromise effect by assuming that, in addition to their deep CPT preferences, participants suffer a loss in utility from choosing a switchpoint farther from the middle row on the screen. Formally, recall that on each screen $q$ of the experiment, a participant makes choices between a fixed prospect, denoted $P_{q f}$, and seven alternatives presented in decreasing order of monetary payoff, denoted $P_{q 1}, P_{q 2}, \ldots, P_{q 7} \cdot{ }^{6}$ Following Hey and Orme (1994), we use a Fechner error specification and assume that on any screen $q$, the participant chooses $P_{q i}$ over $P_{q f}$ if and only if

$$
\frac{U\left(P_{q i}\right)}{\sigma_{q}}+c_{i}+\varepsilon_{q A l t}>\frac{U\left(P_{q f}\right)}{\sigma_{q}}+\varepsilon_{q f} \quad \Longleftrightarrow \quad \varepsilon_{q}<\frac{U\left(P_{q i}\right)-U\left(P_{q f}\right)}{\sigma_{q}}+c_{i}
$$

where $c_{i}$ is a constant that depends on the row $i$ in which the alternative $P_{q i}$ appears, $\sigma_{q}$ is parameter to regulate the relative importance of the utility function vs. the other arguments, and $\varepsilon_{q f}, \varepsilon_{q A l t}$, and $\varepsilon_{q}$ are preference shocks that vary across (but not within) screens. We assume that $\varepsilon_{q f}-\varepsilon_{q A l t} \equiv \varepsilon_{q} \sim N(0,1)$. We refer to $c_{i}$ as the parameter for the compromise effect of row $i$, and we assume that $\Sigma_{i=1}^{7} c_{i}=0$, implying no bias on average toward selecting either the alternative or the fixed prospect. In other words, the constraint implies that this set of parameters does not have an average effect (summing across all rows in the MPL) on the preference between the alternative and the fixed prospect.

Our estimation strategy jointly estimates three sets of parameters: (i) the prospect theory preference parameters for loss aversion, $\lambda$, utility curvature, $\gamma$, and the form of the probability weighting function, $\{\alpha, \beta\}$; (ii) a vector of row-by-row compromise effect parameters, $\left\{c_{i}\right\}_{i=1}^{7}$; and (iii) the scaling parameters, $\sigma_{q}$, that scale utility differences for each screen, $q$. From our perspective, the scaling parameters are nuisance parameters. The incorporation of the (varying) scaling parameters partially addresses the critique of random utility models identified by Wilcox (2011) and Apesteguia and Ballester (2018). Our use of varying scaling parameters follows the spirit of the recommendations of Wilcox (2011). The solution of Apesteguia and Ballester (2018)stochastic preferences parameters - could also be incorporated into our framework, though it would involve substantial computational hurdles because we have four preference parameters.

\footnotetext{
${ }^{6}$ In Part C, the alternative prospects are presented in increasing order of monetary payoff.
} 


\subsection{Estimation}

We estimate the model via Maximum Likelihood Estimation, pooling participants together and clustering the standard errors at the participant level. We impose the parameter restriction $\gamma<1$. 15 of the 28 fixed prospects in Part A have a chance of yielding $\$ 0$ (and likewise for Part B). Accordingly, $\gamma \geq 1$ would imply that any strictly positive alternative sure outcome would be preferred with probability 1. Every participant in the experiment made choices ruling out such extreme risk aversion, except for one participant. ${ }^{7}$

We simplify the estimation in two ways. First, we reduce the number of $\sigma_{q}$ parameters by assuming that $\sigma_{q}$ is identical for screens involving prospects of similar magnitudes. ${ }^{8}$ Second, we assume that $c_{i}$ takes the quadratic functional form $c_{i}=\pi_{0}+\pi_{1} \cdot i+\pi_{2} \cdot i^{2}$. With this functional form, the constraint $\Sigma_{i=1}^{7} c_{i}=0$ implies a linear restriction among the parameters, $\pi_{0}=-4 \pi_{1}-20 \pi_{2}$, so we estimate the two parameters $\pi_{1}$ and $\pi_{2}$.

For each specification, we produce three sets of estimates. First, we estimate $\gamma, \alpha$, and $\beta$ (and the other parameters) with data from all screens from Parts A-D. ${ }^{9}$ To do so, we assume that $\gamma$, $\alpha, \beta$ are the same in the gain and loss domains. Note that $\gamma$ is then the coefficient of relative risk aversion in the gain domain and the coefficient of relative risk seeking in the loss domain. Second, we estimate $\gamma^{+}, \alpha^{+}$, and $\beta^{+}$(and the other parameters) with data from Part A only (which only includes questions in the gain domain and is incentivized). Lastly, we estimate $\gamma^{-}, \alpha^{-}$, and $\beta^{-}$ (and the other parameters) with data from Part B only (which only includes questions in the loss domain).

We exclude from the estimation data participants for whom the MLE algorithm does not converge (after 500 iterations) when the CPT model is estimated separately for each participant with data from Parts A-D. We identified 28 such participants out of a total of 521 participants who

\footnotetext{
${ }^{7}$ As discussed below, we excluded from the estimation participants for whom the MLE did not converge when estimated using only their data. This participant's data were excluded as a result.

${ }^{8}$ For Part A we estimate a $\sigma_{q}$ parameter for each of five groups of screens. Screens are grouped together based on the expected utility of their fixed prospects; the latter is calculated based on the parameter estimates reported by Fehr-Duda and Epper (2012, Table 3). We estimate $\sigma_{A, 0-25}, \sigma_{A, 25-50}, \sigma_{A, 50-75}, \sigma_{A, 75-100}, \sigma_{A, 100+}$, where $\sigma_{A, L-H}$ is for screens with a fixed prospect whose expected value is between $L$ and $H$. For Part $\mathrm{B}$, we proceed analogously. We also estimate $\sigma_{C \text {,small }}$ and $\sigma_{C \text {,big }}$ for the two smaller and the two larger fixed prospects of Part C, respectively, and $\sigma_{D}$ for the two fixed prospects of the two screens of Part D we use.

${ }^{9}$ We drop the two screens of Part D that involve only positive outcomes (designed by T\&K as placebo tests for loss aversion) so that Parts C and D primarily identify $\hat{\lambda}$. When we refer to "all screens from Parts A-D," we mean all screens excluding these two.
} 
completed all parts of the experiment, and most of them had haphazard response patterns.

To derive a likelihood function, first recall that the experimental procedure constrained participants to behave consistently: if a participant chooses $P_{q i}$ over $P_{q f}$ for some $i>1$, then the participant chooses $P_{q j}$ over $P_{q f}$ for all $j<i$. Hence the probability that the participant switches from choosing the alternative when the alternative is $P_{q i}$ to choosing the fixed prospect when the alternative is $P_{q(i+1)}$ is

$$
\begin{aligned}
\operatorname{Pr}_{q, i, i+1} & \equiv \operatorname{Pr}\left(\text { participant switches between } P_{q i} \text { and } P_{q(i+1)}\right) \\
& =\operatorname{Pr}\left(\frac{U\left(P_{q(i+1)}\right)-U\left(P_{q f}\right)}{\sigma_{q}}+c_{i+1}<\varepsilon_{q}<\frac{U\left(P_{q i}\right)-U\left(P_{q f}\right)}{\sigma_{q}}+c_{i}\right) \\
& =\Phi\left(\frac{U\left(P_{q i}\right)-U\left(P_{q f}\right)}{\sigma_{q}}+c_{i}\right)-\Phi\left(\frac{U\left(P_{q(i+1)}\right)-U\left(P_{q f}\right)}{\sigma_{q}}+c_{i+1}\right),
\end{aligned}
$$

where $\Phi(\cdot)$ is the CDF of a standard normal random variable; the probability that the participant always chooses the fixed prospect is $\operatorname{Pr}_{q,-, 1} \equiv 1-\Phi\left(\left(U\left(P_{q 1}\right)-U\left(P_{q f}\right)\right) / \sigma_{q}+c_{1}\right)$; and the probability that the participant always chooses the alternative over the fixed prospect is $\operatorname{Pr}_{q, 7,-} \equiv \Phi\left(\left(U\left(P_{q 7}\right)-\right.\right.$ $\left.\left.U\left(P_{q f}\right)\right) / \sigma_{q}+c_{7}\right)$. We assume that $\varepsilon_{q}$ is drawn i.i.d. for each screen $q$ in the set of screens, $Q$, faced by a participant.

Thus, the likelihood function for any given participant $p$ is:

$$
L_{p}=\prod_{q \in Q} \prod_{i=0,1, \ldots, 7}\left(\operatorname{Pr}_{q, i, i+1}\right)^{1\left\{p \text { switches between } P_{q i} \text { and } P_{q, i+1}\right\}}
$$

where, for notational simplicity, we write $\operatorname{Pr}_{q, 0,1}$ for $\operatorname{Pr}_{q,-, 1}$ and $\operatorname{Pr}_{q, 7,8}$ for $\operatorname{Pr}_{q, 7,-}$. The likelihood function for all the participants pooled together is $\Pi_{p \in P} L_{p}$, where $P$ is the set of participants.

\subsection{Robustness checks}

In addition to the baseline CPT model described above (with CRRA utility and the Prelec (1998) probability weighting function), we estimated three additional models: (1) the CPT model with CRRA utility but with T\&K's probability weighting function: $\omega(p)=p^{\alpha} /\left(p^{\alpha}+(1-p)^{\alpha}\right)^{\frac{1}{\alpha}} ;(2)$ the CPT model with the Prelec probability weighting function, but with CARA (a.k.a. "exponential") utility (Köbberling and Wakker 2005), $u(x)=\frac{1-e^{-\alpha_{\text {expo }}^{+} x}}{\alpha_{\text {expo }}^{+}}$if $x \geq 0, u(-x)=\frac{1-e^{-\alpha_{\text {expo }}^{-}|x|}}{\alpha_{\text {expo }}^{-}}$if $x<0$; and (3) the CPT model with the Prelec probability weighting function, but with expo-power utility 
(Saha 1993), $u(x)=\frac{1-e^{-\alpha_{\mathrm{e}-\mathrm{p}} x^{1-\gamma_{\mathrm{e}-\mathrm{p}}}}}{\alpha_{\mathrm{e}-\mathrm{p}}}$. The results presented below in Sections 5 and 6 are robust to the use of these alternative models (see the Online Appendix for details).

\subsection{Identification With and Without the Pull Treatments}

Our five Pull treatments are designed to identify the effect of the compromise effect on measured risk preferences. However, even without the Pull treatments, generic risk aversion experiments will be able to identify the compromise effect parameters. To gain intuition for this fact, consider a MPL experiment in which each screen features a different level of risk aversion that elicits indifference at the middle row of the MPL. Accordingly, measured risk aversion will vary across screens (unless the researcher takes account of the compromise effect). Hence, the compromise effect will be identified as long as (i) the compromise-effect parameters are included in the model, and (ii) the level of risk aversion that elicits indifference in the middle row varies across MPL screens. Because the compromise effect parameters would be identified even without within-subject variation in the Pull treatment, our data could also be used to identify the compromise effects at the level of each individual participant, but those estimates would be less precise than the representative agent estimates on which we focus in this paper.

\section{Hypotheses}

Having defined the model, we now articulate a number of hypotheses that we will test empirically by estimating the model with the data from the experiment. Drawing on prior work (see the Introduction for discussion), our starting point is the hypothesis that participants will be biased toward switching close to the middle of the seven rows in the Multiple Price List.

Hypothesis 1: Estimates of $c_{i}$ will reveal a compromise effect. Specifically, $\hat{c}_{i}$ will be positive in the top rows, close to zero in the middle rows, and negative in the bottom rows, decreasing monotonically from the first to the last row.

Note that a positive value of $c_{i}$ implies a bias in favor of choosing the alternative (which is in the right-hand-side column of the MPL), and a negative value of $c_{i}$ implies a bias in favor of choosing 
the fixed prospects (which is in the left-hand-side column of the MPL). So Hypothesis 1 implies a switch point that is biased toward the middle row of the MPL.

Thus, the compromise effect implies that measured risk aversion in the gain domain, as assessed in Part A, will be systematically increased across the range of treatments from Pull -2 to Pull 2 (in the model without the compromise effect). For instance, consider the two example screenshots from the Introduction. The first screenshot illustrates the Pull -2 treatment. Since the intermediate alternatives are shifted away from zero, the compromise effect induces participants to choose an indifference point that is farther from zero, thereby implying a relatively low level of risk aversion. In contrast, in the Pull 2 treatment, illustrated in the second screenshot, the intermediate alternatives are shifted closer to zero. The compromise effect causes participants to choose an indifference point that is closer to zero, thereby implying a relatively high level of risk aversion.

The hypothesized effect of the Pull treatments on measured risk seeking in the loss domain is analogous. Moving across the range of treatments from Pull -2 to Pull 2 is now hypothesized to raise estimated risk seeking. For example, consider a fixed prospect that has outcomes in the loss domain. In the Pull -2 treatment, the intermediate alternatives are all negative and shifted away from zero, coaxing participants to choose an indifference point that is farther from zero, thereby implying a relatively low level of risk seeking. By contrast, in the Pull 2 treatment, the intermediate alternatives are all negative and shifted relatively close to zero, coaxing participants to choose an indifference point that is closer to zero, thereby implying a relatively high level of risk seeking.

Similar considerations imply that moving across the range of treatments from Pull -2 to Pull 2 is predicted to reduce the level of estimated loss aversion.

We thus hypothesize that the compromise effect affects estimates of risk aversion and loss aversion in the traditional CPT model. In Section 3.2 above, we introduced a model that incorporates parameters for the compromise effect. If that model is properly specified, we would expect the bias induced by the compromise effect to disappear and the estimates of risk aversion and loss aversion to be similar across Pull treatments. In summary, we hypothesize:

Hypothesis 2.a: Estimates of relative risk aversion in the gain domain $\left(\gamma, \gamma^{+}\right)$and relative risk seeking in the loss domain $\left(\gamma, \gamma^{-}\right)$from our model with the compromise effect will not vary in Pull.

Hypothesis 2.b: Estimates of loss aversion $(\lambda)$ from our model with the compromise effect will not 
vary in Pull.

Hypothesis 3.a: Estimates of $\gamma, \gamma^{+}$, and $\gamma^{-}$from the model without the compromise effect will be increasing in Pull.

Hypothesis 3.b: Estimates of $\lambda$ from the model without the compromise effect will be decreasing in Pull.

\section{Estimating the Compromise Effect and Risk Preferences Jointly}

We begin by estimating our model with the compromise effect. We focus our attention on the curvature parameter $\gamma$ and the loss aversion parameter $\lambda$ because our ex ante hypotheses are about these parameters. We do not interpret the results for the other parameters $(\alpha, \beta$, and the $\sigma_{q}$ parameters) because we did not have ex ante hypotheses, but we report the estimates for all parameters in the Online Appendix.

Table 1 shows the estimates for our parameters of interest. The estimates of $\gamma$ (obtained from the data from all parts together), $\gamma^{+}$(obtained from the data from Part A only), and $\gamma^{-}$(obtained from the data from Part B only) differ substantially from one another, ranging from $\hat{\gamma}^{-}=-0.106$ to $\hat{\gamma}^{+}=0.448$. The estimate of $\gamma^{-}$is significantly smaller than 0 at the $5 \%$ level, indicating risk aversion in the loss domain, which is the opposite of what CPT predicts. The estimate of $\lambda$ (obtained from the data from all parts together) is 1.311, consistent with some loss aversion, albeit less than usually assumed. Except for the notably small estimate of $\gamma^{-}$, our parameter estimates (including those for the probability weighting function parameters) are broadly in line with existing estimates in the literature. We compare our estimates to the literature in Section 10.

\section{$<$ INSERT TABLE 1 ABOUT HERE $>$}

The sizeable difference between the estimates in Parts A and B suggests that the assumption that $\gamma, \alpha$, and $\beta$ are the same in the gain and loss domains is unsupported by the data. We nonetheless maintain this assumption when estimating the model with the data from all parts of

the experiment because we are interested in studying $\hat{\lambda}$, and as Wakker (2010) points out, assuming 
different parameters in the gain and loss domains makes the loss aversion parameter more difficult to interpret. ${ }^{10}$

\subsection{Estimating the Compromise Effect}

We now proceed to test Hypothesis 1, which predicts that the parameters for the compromise effect $c_{i}$ will be positive in the top rows, close to zero in the middle rows, and negative in the bottom rows, and will decrease from the first to the last row.

The estimated $c_{i}$ 's are calculated from the estimates of $\pi_{1}$ and $\pi_{2}$. Figure 2 shows the estimated $c_{i}$ for each row $i$ (the numerical values are listed in the Online Appendix). As can be seen, the estimated $c_{i}$ 's decline from row 1 (where $c_{1}$ is large and positive) to row 7 (where $c_{7}$ is large and negative), and $c_{4}$ is always relatively small (in fact, it is not significantly different from 0 at the $5 \%$ level when estimated with the data from Part A or Part B only). These results indicate that participants tend to switch from choosing the alternative to choosing the fixed prospect toward the middle row. Furthermore, the estimates of the $\pi_{1}$ and $\pi_{2}$ parameters reported in Table 1 are highly jointly statistically distinguishable from zero: the $p$-value of the Wald test is less than $1 \times 10^{-10}$. These results strongly support Hypothesis 1 and are robust to restricting the data to the incentivized Part A only. We note that the compromise effect is weaker when estimated with the data from Part A versus the data from Part B. This may suggest that the compromise effect is stronger in the loss domain; alternatively, participant fatigue and the lack of incentives in Part B could have led to reduced participant attention and to a stronger compromise effect.

\footnotetext{
${ }^{10}$ Wakker $\left(2010\right.$, section 9.6) highlights two concerns when $u(\cdot)$ takes the CRRA form and $\gamma^{+} \neq \gamma^{-}$. First, the ratio of disutility from a sure loss of $x$ to utility from a sure gain of $x, \frac{-\lambda u^{-}(-x)}{u^{+}(x)}$, is not uniformly equal to $\lambda$ but instead depends on the value of $x$. Second, for any $\lambda$, there exists a range of $x$ values for which this ratio is actually smaller than 1, which is the opposite of loss aversion. These problems make estimates of $\lambda$ sensitive to exactly which prospects are used in the experiment. As previously mentioned, in the Online Appendix we report estimates of a robustness check where we assume CARA utility and different risk aversion parameters in the gain and loss domains.
} 


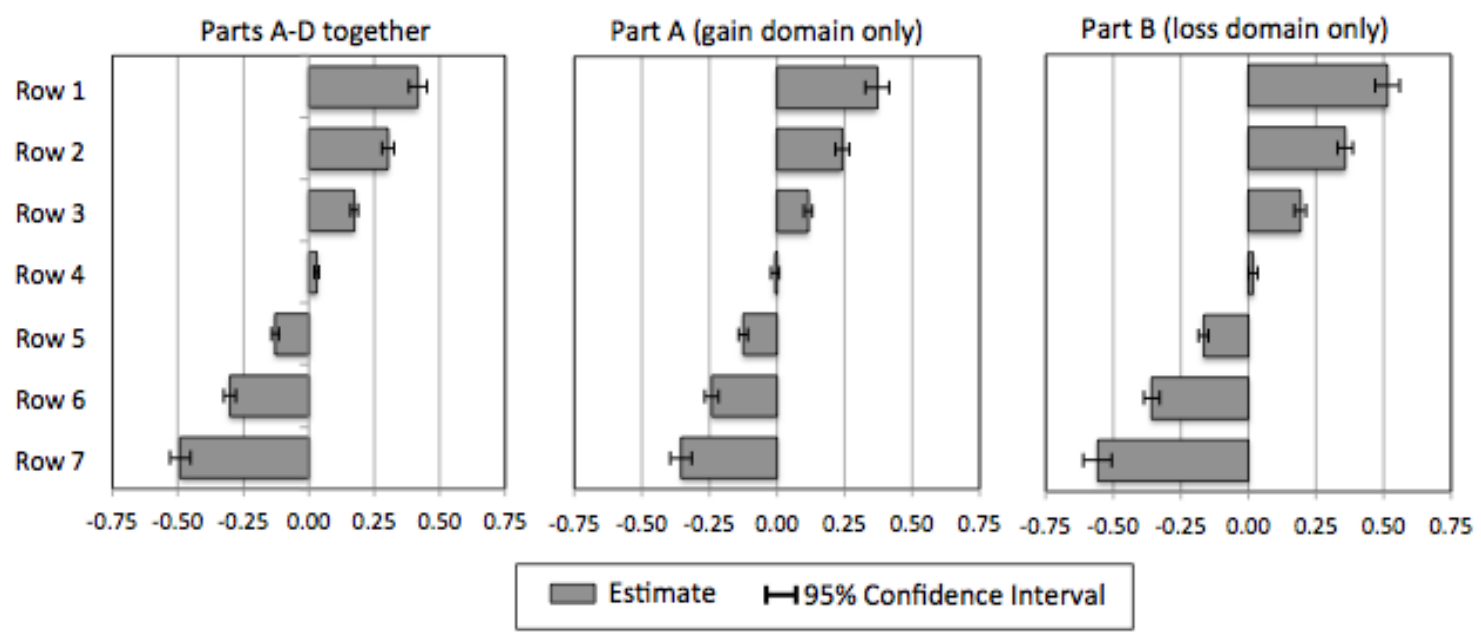

FIGURE 2. Implied estimates of the parameters for the compromise effect $c_{i}$ as a function of the row $i$ in which a choice appears. In the estimation, we parameterize the parameters for the compromise effect with the quadratic functional form $c_{i}=\pi_{0}+\pi_{1} \cdot i+\pi_{2} \cdot i^{2}, \Sigma_{i=1}^{7} c_{i}=0$, which is equivalent to $c_{i}=\pi_{1} \cdot(i-4)+\pi_{2} \cdot\left(i^{2}-20\right)$. Note that the confidence intervals are smaller around the middle rows because $\operatorname{var}\left(\hat{c}_{i}\right) \approx(i-4)^{2} \operatorname{var}\left(\hat{\pi}_{1}\right)+\left(i^{2}-20\right)^{2} \operatorname{var}\left(\hat{\pi}_{2}\right)\left(\operatorname{assuming} \operatorname{cov}\left(\hat{\pi}_{1}, \hat{\pi}_{2}\right) \approx 0\right)$.

\subsection{Robustness of the Preference-Parameter Estimates from Joint Estimation}

To test Hypotheses $2 \mathrm{a}$ and $2 \mathrm{~b}$, we begin by estimating the model with the compromise effect separately in the subsamples corresponding to each of the five Pull treatments. Figure 3 shows estimates of $\gamma, \gamma^{+}$and $\gamma^{-}$, with $95 \%$ confidence intervals, for each subsample. Figure 4 shows estimates of $\lambda$. 

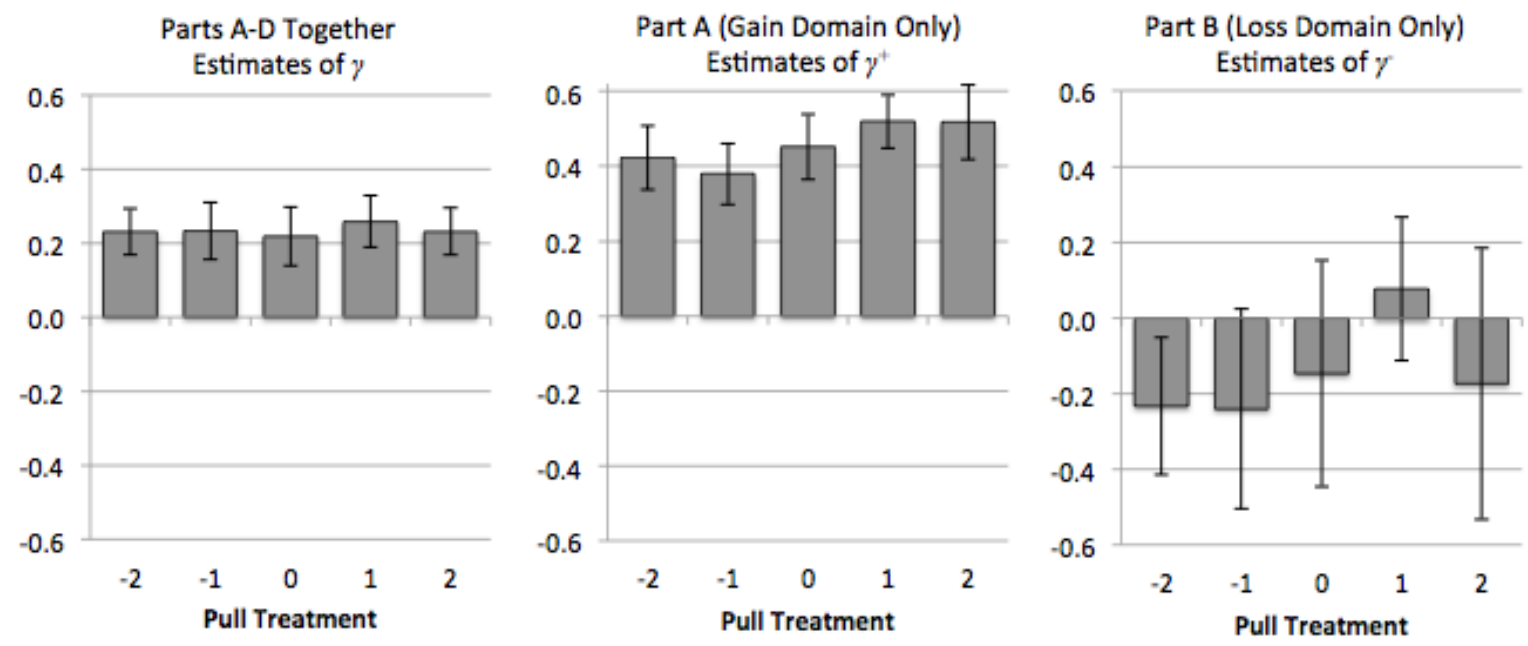

Estimate

$\mapsto 95 \%$ Confidence Interval

FIGURE 3. Estimates of $\gamma, \gamma^{+}$, and $\gamma^{-}$by Pull treatment, from the model with the compromise effect. The negative estimates of $\gamma^{-}$for Part B reflect risk aversion in the loss domain, unlike what CPT predicts. ( $\gamma$ is not estimated for Parts $\mathrm{C}$ and $\mathrm{D}$ only because these parts have few questions.)

As can be seen, the estimates of $\gamma, \gamma^{+}, \gamma^{-}$, and $\lambda$ do not differ substantially across Pull treatments, consistent with Hypotheses $2 \mathrm{a}$ and $2 \mathrm{~b}$. To formally test for equality across treatments, we estimate the model with all parameters specified as linear functions of the Pull variable and of a dummy that indicates if the participant was in the EV treatment. In other words, we substitute $\gamma$ in the utility function in (1) by $\gamma=\gamma_{0}+\phi_{1}^{\gamma} \cdot P u l l+\phi_{2}^{\gamma} \cdot E V, \lambda$ by $\lambda=\lambda_{0}+\phi_{1}^{\lambda} \cdot P u l l+\phi_{2}^{\lambda} \cdot E V$, and do likewise for $\alpha, \beta$, and all the $\sigma_{q}$ parameters, and we test whether the $\phi$ parameters are equal to zero. $^{11}$

\footnotetext{
${ }^{11}$ The statistical power to test the pairwise differences in our parameter estimates (for each discrete step in the Pull treatment) is limited. Accordingly, we test Hypothesis 3.a and Hypothesis 3.b by estimating a linear model. Figures 5 and 6 imply that a linear specification is a good approximation.
} 


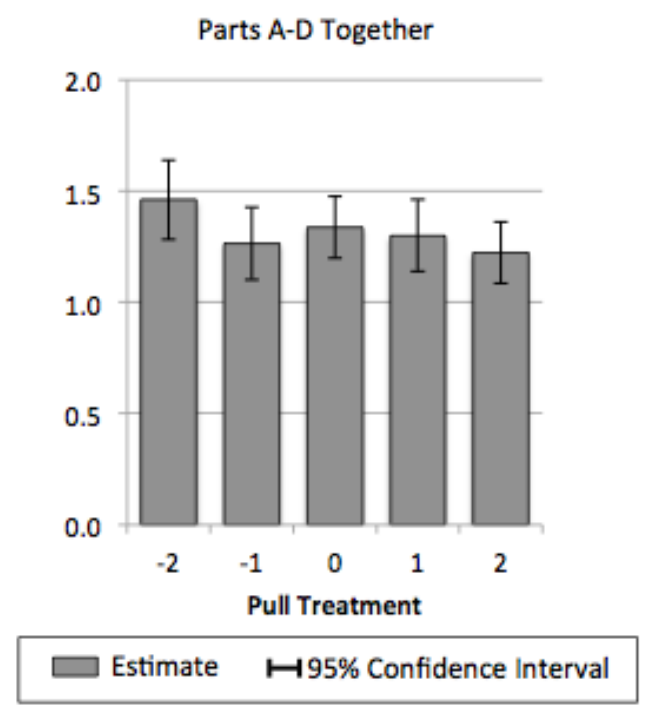

FIGURE 4. Estimates of $\lambda$ by Pull treatment from the model with the compromise effect, for Parts A-D together. ( $\lambda$ cannot be estimated for Part A only or Part $\mathrm{B}$ only because the questions in these parts are all in the gain or loss domains. We do not estimate $\lambda$ for Parts $\mathrm{C}$ and D only because these parts have few questions.)

Table 2 shows the results. The three estimates of $\phi_{1}^{\gamma}$ are all close to zero, and none is statistically distinguishable from zero (including the estimate from the incentivized Part A). We interpret these estimates as providing more formal support for Hypothesis 2a. The estimate of $\phi_{1}^{\lambda}$ is significantly different from zero at the $10 \%$ level, and its sign is consistent with what one would expect from the Pull manipulation, which suggests that our model with the compromise effect does not perfectly control for this effect. As we will see below, however, this estimate of $\phi_{1}^{\lambda}$ is much smaller than the one obtained from the model without the compromise effect, indicating that our model with the compromise effect substantially reduces the bias due to this effect.

\section{$<$ INSERT TABLE 2 ABOUT HERE $>$}

Taken together, we interpret the evidence as strongly supportive of Hypothesis 2a and also broadly supportive of Hypothesis $2 \mathrm{~b}$. In other words, our model (2) yields robust estimates of the CPT parameters $\gamma$ and $\lambda$, both when estimated in the sample of all participants and within the subsamples corresponding to each of the five Pull treatments. 


\section{Biases in Estimated Risk Preferences when the Compromise Effect Is Omitted from the Model}

We now proceed to estimate the CPT model without the compromise effect, the version of the model usually estimated by economists. As above, we focus our attention on $\gamma$ and $\lambda$; results for all parameters are presented in the Online Appendix.

Table 3 shows the estimates for selected parameters. The estimates of $\gamma, \gamma^{+}$and $\gamma^{-}$are all smaller in magnitude than those from the model with the compromise effect (2), indicating less curvature in the utility function. The estimate of $\gamma^{-}$is not significantly different from 0 anymore, consistent with a linear utility function in the loss domain. The estimate of $\lambda$ is not significantly different from its value when estimated in the model with the compromise effect.

\section{$<$ INSERT TABLE 3 ABOUT HERE $>$}

The parameter estimates all fall within the range of existing estimates in the literature (except for $\hat{\beta}^{+}$, which falls slightly below the range).

To test Hypotheses 3a and 3b, we proceed analogously as above and estimate the model without the compromise effect separately in the subsamples corresponding to each of the five Pull treatments. As can be seen from Figures 5 and 6, the estimates differ substantially across Pull treatments. As predicted by Hypotheses $3 \mathrm{a}$ and $3 \mathrm{~b}, \hat{\gamma}, \hat{\gamma}^{+}$and $\hat{\gamma}^{-}$are increasing in Pull and $\hat{\lambda}$ is decreasing in Pull. Comparing Figures 5 and 6 to Figures 3 and 4, it is clear that failing to control for the compromise effect when estimating the model separately for each treatment introduces a sizeable bias in the estimates of $\gamma$ and $\lambda$.

As can be seen in the right panel of Figure 5, the Pull treatment manipulation of the compromise effect is strong enough to generate estimates of $\gamma^{-}$that are either significantly smaller than 0 (Pull -2) or significantly larger than 0 (Pull 2). Furthermore, as can be seen from Figure 6, the Pull treatment manipulation of the compromise effect causes estimates of $\lambda$ to vary from 1.059 (Pull 2) to 1.746 (Pull -2). The former estimate is not significantly different from 1 at the $10 \%$ level, suggesting that the compromise effect can create the appearance of no loss aversion. 

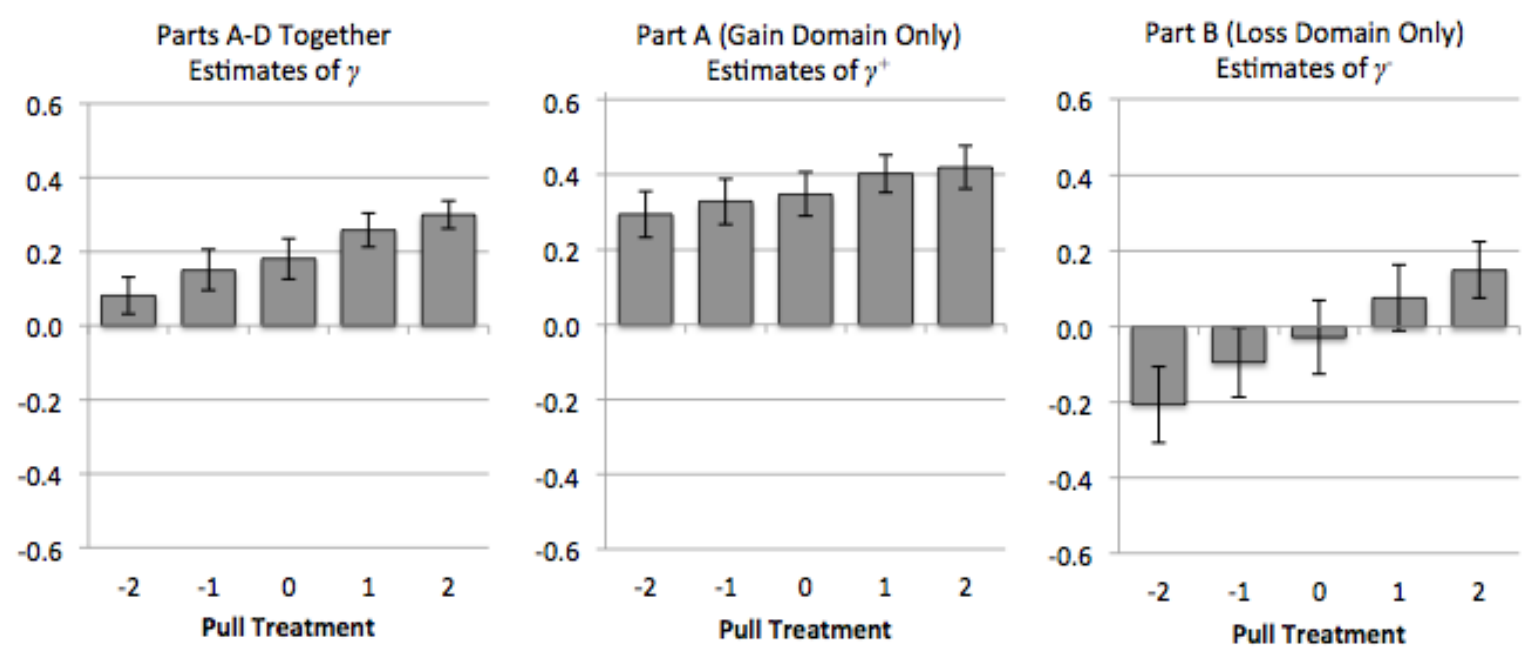

Estimate $\mapsto 95 \%$ Confidence Interval

FIGURE 5. Estimates of $\gamma, \gamma^{+}$, and $\gamma^{-}$by Pull treatment, from the model without the compromise effect. This figure is analogous to Figure 3, except that the estimated model does not control for the compromise effect.

As above, we formally test the impact of the compromise effect by specifying all parameters as linear functions of the Pull variable and of a dummy that indicates if the participant was in the EV treatment. The results are presented in Table 4. $\hat{\phi}_{1}^{\gamma}$ is significant at the $1 \%$ level and positive in all three columns (including in the column corresponding to the incentivized Part A), providing formal support for Hypothesis 3a. The implied differences between the estimates in the Pull -2 and the Pull 2 treatments are sizeable: for $\hat{\gamma}$, the implied difference is $0.168(4 \times 0.042)$, and for $\hat{\gamma}^{-}$, the corresponding figure is $0.252(4 \times 0.063) . \hat{\phi}_{1}^{\lambda}$ is highly statistically significant and negative, thus supporting Hypothesis 3b. The implied difference between $\hat{\lambda}$ in the Pull -2 and the Pull 2 treatments is $0.588(4 \times 0.147)$. 


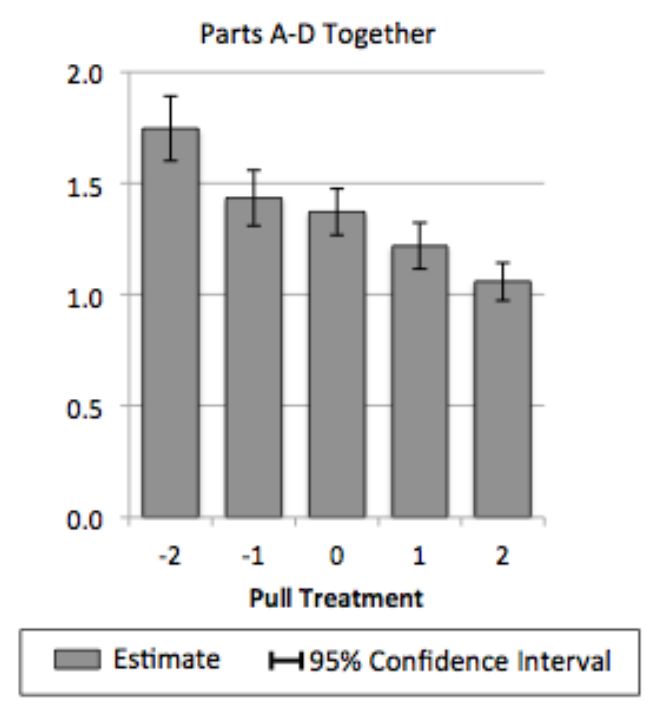

FIGURE 6. Estimates of $\lambda$ by Pull treatment from the model without the compromise effect, for Parts A-D together. This figure is analogous to Figure 4, except that the estimated model does not control for the compromise effect.

\section{$<$ INSERT TABLE 4 ABOUT HERE $>$}

The evidence thus strongly supports Hypotheses $3 \mathrm{a}$ and $3 \mathrm{~b}$ and suggests that many existing results based on experiments using the MPL elicitation method may be severely biased due to the compromise effect.

\section{How Large is the Compromise Effect?}

Having demonstrated that the compromise effect can have a significant impact on choice in a MPL setting, we now obtain a rough estimate of its importance relative to the prospects' monetary outcomes.

To do so, we make an assumption that we show in the next paragraph is justified empirically: the magnitude of the compromise effect and of the preference shocks scales linearly with the expected utilities of the prospects on a screen. Formally, we assume that there is a constant $\Delta>0$ such that 
for all screens $q$,

$$
\sigma_{q}=\Delta \cdot\left|U\left(P_{q f}\right)\right|
$$

where the parameter $\sigma_{q}$ (as defined in Section 3.2) regulates the relative importance of utility vs. the other parameters for the compromise effect and shocks, and $U\left(P_{q f}\right)$ is the expected utility of the fixed prospect on screen $q$. Thus, for the prospects from Part A (which are all in the gain domain, allowing us to ignore the absolute value sign), we can substitute $\Delta \cdot U\left(P_{q f}\right)$ for $\sigma_{q}$ in Equation (2) of our model. It follows that a participant will prefer the alternative $P_{q i}$ over the fixed prospect $P_{q f}$ in row $i$ of screen $q$ if and only if

$$
\begin{aligned}
& U\left(P_{q i}\right)-U\left(P_{q f}\right)+\Delta \cdot c_{i} \cdot U\left(P_{q f}\right)>\sigma_{q} \varepsilon_{q} \\
\Longleftrightarrow & U\left(P_{q i}\right)-U\left(\left(1+\theta_{i}\right) \cdot P_{q f}\right)>\sigma_{q} \varepsilon_{q},
\end{aligned}
$$

where $\left(1+\theta_{i}\right)=\left(1-\Delta c_{i}\right)^{\frac{1}{1-\gamma}}$. For the prospects from Part B, a similar equivalence holds, but with $\left(1+\theta_{i}\right)=\left(1+\Delta c_{i}\right)^{\frac{1}{1-\gamma}}$. Therefore, our assumption enables us to quantify the influence of a compromise effect $c_{i}$ as the factor $\left(1+\theta_{i}\right)$ by which the screen's fixed prospect would have to be multiplied to have the same effect on choice. Equivalently, $\theta_{i}$ is the magnitude of the compromise effect measured in terms of a fraction of monetary value of the screen's fixed prospect (with a negative value meaning that the compromise effect makes the fixed prospect less likely to be chosen).

We now assess our assumption in equation (3) empirically. Recall from Section 3.3 that, to estimate our models, we group screens together that have similar expected values of their fixed prospects and estimate a common $\hat{\sigma}_{q}$ for each group. Defining (and slightly abusing) some notation, let $\hat{U}\left(P_{\tilde{q} f}\right)$ denote the expected utility of the fixed prospect on screen $\tilde{q}$ calculated using the model parameters estimated from the specification with the compromise effect; and let $\hat{E}_{\tilde{q} \in q}\left[\left|\hat{U}\left(P_{\tilde{q} f}\right)\right|\right]$ denote the mean of the absolute values of these $\hat{U}\left(P_{\tilde{q} f}\right)$ 's across all the screens $\tilde{q}$ in group $q$. (Because the screens in a group have similar $\hat{U}\left(P_{\tilde{q} f}\right)$ 's, each $\hat{U}\left(P_{\tilde{q} f}\right)$ has roughly the same magnitude as the group mean.) The empirical counterpart to equation (3) would be a multiplicative relationship between $\hat{\sigma}_{q}$ and $\hat{E}_{\tilde{q} \in q}\left[\left|\hat{U}\left(P_{\tilde{q} f}\right)\right|\right]$ that is the same across different groups $q$. Figure 7 illustrates this relationship in our data. As can be seen, for the three sets of estimation results (Parts A-D together, 
Part A, and Part B), $\hat{\sigma}_{q}$ indeed appears to be reasonably well approximated as a multiplicative constant times $\hat{E}_{\tilde{q} \in q}\left[\left|\hat{U}\left(P_{\tilde{q} f}\right)\right|\right]$. Moreover, the multiplicative constant $\hat{\Delta}$ is nearly the same across the three sets of results, ranging from 0.32 to $0.36 .^{12}$
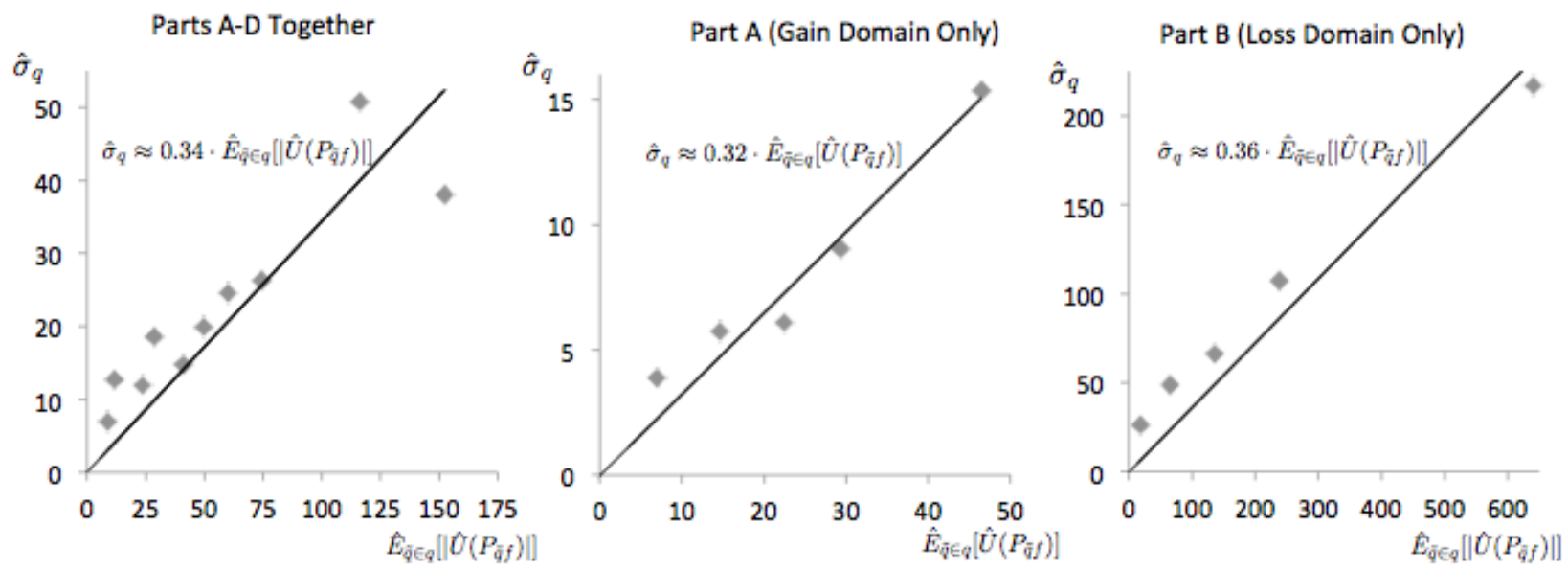

FIGURE 7. Relationship between $\hat{\sigma}_{q}$ and the expected utility of a screen's fixed prospect. See text for details.

Using the estimated $\hat{\Delta}$ for each of the three sets of results, Table 5 presents estimates of the strength of the compromise effect, $\hat{\theta}_{i}$, for each row $i$ on a screen (because this is meant to be an approximation, we omit standard errors).

\section{$<$ INSERT TABLE 5 ABOUT HERE $>$}

Our estimates of the strength of the compromise effect in a screen's first and last rows (where their impact is largest) range in magnitude from $\sim 17 \%$ to $\sim 23 \%$ of the monetary value of the screen's fixed prospect. We interpret such magnitudes as non-trivial.

\footnotetext{
${ }^{12}$ In OLS regressions of $\hat{\sigma}_{q}$ on a constant and $\hat{E}_{\tilde{q} \in q}\left[\left|\hat{U}\left(P_{\tilde{q} f}\right)\right|\right]$, the intercept is economically small in all cases. For the estimates of $\hat{\Delta}$ reported here, we use a 0 intercept.
} 


\section{Demographic Correlates of the CPT Model Parameters and of the Parameters that Capture the Compromise Effect}

A large literature seeks to estimate the demographic correlates of economic preferences and decision making (e.g., Beauchamp, Cesarini, and Johannesson 2017, Benjamin, Brown, and Shapiro 2013, Booij, van Praag, and van de Kuilen 2010, Dohmen, Falk, Huffman, and Sunde 2010). The data we collected in our experiment, which include a number of demographic variables, allow us to contribute to this literature by analyzing the demographic correlates of the four key parameters of the CPT model $(\gamma, \lambda, \alpha, \beta)$ and of the two model parameters that capture the compromise effect $\left(\pi_{1}\right.$, $\left.\pi_{2}\right)$. In our baseline demographic specification, we estimate our CPT model with the compromise effect using data from Parts A-D together, with these six key model parameters specified as linear functions of a constant, age, sex, a dummy variable indicating whether one has a college degree, SAT Math score, the log of one's parents' combined annual income, as well as dummy variables to control for race. We also estimated several additional specifications to verify the robustness of the results from our baseline demographic specification. First, we estimated the baseline demographic specification again, but using data from Part A only, and then using data from Part B only. Second, we estimated a specification akin to the baseline demographic specification using data from Parts AD together, but with CARA (a.k.a. "exponential") utility (Köbberling and Wakker 2005). Lastly, we employed a two-step procedure in which we first estimated our baseline CPT model with the compromise effect separately for each participant, and then regressed each estimated parameter of interest on the demographic variables. To reduce the number of parameters and thereby improve the frequency of convergence in the first step of that procedure, we assume that $\sigma_{q}$ is identical across all screens (for each experimental participant).

Two main results stand out across the baseline and robustness specifications. First, higher SAT Math scores are associated with lower $\gamma$-i.e., with lower risk aversion in the gain domain and higher risk aversion (or, equivalently, lower risk seeking) in the loss domain. This result is consistent with the existing literature on the association between cognitive ability and risk preferences (see Dohmen, Falk, Huffman, and Sunde 2018 for a review of the literature), although it has been argued that this association is driven by the fact that measurement noise may be higher for individuals with lower cognitive ability (Andersson, Holm, Tyran, and Wengström 2016). The second result that 
stands out is that higher SAT Math scores are associated with higher loss aversion $(\lambda)$. This result, although robust across our specifications, is surprising given that previous research has found that education is negatively associated with loss aversion (Booij, van Praag, and van de Kuilen 2010, Gächter, Johnson, Herrmann 2007, Hjorth and Fosgerau 2011). Aside from these two results, the associations between the other covariates and parameters were not statistically distinguishable from zero or were not robust across specifications.

The Online Appendix reports estimates of the baseline demographic specification and provides additional details. We note that one limitation of this analysis is that our sample of experimental participants was not selected to be representative of the population.

\section{Effect of Displaying the Gambles' Expected Values on Esti- mated Risk Preferences}

Displaying the expected value may anchor the participants on the expected value (Tversky and Kahneman, 1974) or simplify comprehension of the gamble (Benjamin, Brown, and Shapiro, 2013), thereby making observed preferences more risk neutral. We therefore hypothesize that $(1) \hat{\gamma}^{+}$and $\hat{\gamma}^{-}$will shift toward 0 in the EV treatment, and (2) $\hat{\lambda}$ will shift toward 1 in the EV treatment.

Online Appendix Figures 1-4 show estimates of $\hat{\gamma}$ and $\hat{\lambda}$ for the subsamples corresponding to the two EV treatments, with $95 \%$ confidence intervals. Displaying the expected value does not appear to affect estimated risk preferences or loss aversion. In addition, none of the estimates of $\hat{\phi}_{2}^{\gamma}$ and of $\hat{\phi}_{2}^{\lambda}$ in Table 4 are statistically distinguishable from zero. Thus, like Lichtenstein, Slovic, and Zink (1969) and Montgomery and Adelbratt (1982) but unlike Harrison and Rutström (2008), we do not find support for the hypothesis that the EV treatment shifts $\hat{\gamma}^{+}$and $\hat{\gamma}^{-}$toward 0 and $\hat{\lambda}$ toward 1. A difference between our experiment and Harrison and Rutström's (2008) is that the prospects in the latter are more complex, involving four possible outcomes. It is possible that participants intuitively estimate the prospects' expected values in our experiment but are not able to accurately do so in Harrison and Rutström's experiment, and that providing expected value information is therefore redundant in our experiment but not in theirs. 


\section{Conclusion}

In this paper, we estimate an econometric model that explicitly takes into account the compromise effect and thus disentangles it from risk preference parameters. The resulting risk-preference estimates are robust: the inferred risk parameters essentially do not change with exogenous manipulations of the compromise effect. Without parameters for the compromise effect, however, we replicate the finding from prior work that risk-preference estimates are sensitive to exogenous manipulations of the compromise effect.

How do our "debiased" preference-parameter estimates (from Table 1) compare to those from the literature? For risk aversion in the gain and loss domains, we estimate $\hat{\gamma}^{+}=0.448$ and $\hat{\gamma}^{-}=-0.106$, respectively. Booij, van Praag, and van de Kuilen's (2010) Table 1 reviews existing experimental estimates. Translated into the CRRA functional form we estimate, the range of existing parameter estimates is $\hat{\gamma}^{+} \in[-0.01,0.78]$ in the gain domain and $\hat{\gamma}^{-} \in[-0.06,0.39]$ in the loss domain. For loss aversion, we estimate $\widehat{\lambda}=1.311$. Although T\&K estimated $\lambda$ to be 2.25 , the literature contains a broad range of estimates: among the papers reviewed by Abdellaoui, Bleichrodt, and Paraschiv (2007, Tables 1 and 5), $\hat{\lambda} \in[0.74,8.27]$, and among those reviewed by Booij, van Praag, and van de Kuilen (2010, Table 1), $\hat{\lambda} \in[1.07,2.61]$. Finally, our estimates of the two-parameter Prelec (1998) probability-weighting parameters are in the ranges $\widehat{\alpha} \in[0.564,0.690]$ and $\widehat{\beta} \in[0.858,1.471]$. Booij, van Praag, and van de Kuilen's (2010) Table 1 only lists three studies that estimated this functional form, and they only did so for prospects in the gain domain. The ranges of estimates are $\hat{\alpha}^{+} \in[0.53,1.05]$ and $\hat{\beta}^{+} \in[1.08,2.12]$. Fox and Poldrack's (2014) Table A.3 also lists three studies that estimated the two-parameter Prelec (1998) functional form for prospects in the gain domain. The ranges of estimates are $\hat{\alpha}^{+} \in[0.62,1.15]$ and $\hat{\beta}^{+} \in[1.00,1.58]$. Overall, then, our parameter estimates are broadly in line with existing estimates in the literature, except that some of our estimates of the probability weighting parameter $\beta^{+}$and our estimate of risk aversion in the loss domain $\gamma^{-}$fall below the range of estimates in the literature -indeed, our estimate of $\gamma^{-}$ indicates risk aversion in the loss domain, the opposite of CPT's prediction.

As in $\mathrm{T} \& \mathrm{~K}$, our estimation of the prospect-theory parameters has assumed that the reference point is the participant's status-quo wealth. Köszegi and Rabin (2006, 2007) have argued that the assumption that the reference point is the participant's (possibly stochastic) expectation of 
wealth provides a better explanation of risk-taking behavior in a variety of contexts. Could a version of prospect theory in which the reference point reflects a participant's expectations explain why the manipulations of the choice set influence the estimated preference parameters (when we do not include parameters for the compromise effect)? This question poses a challenging research program. Modeling the reference point as an expectation would not merely make the reference point depend on the alternative options in the current choice problem but also on the sequence of choice problems that have been faced already, as well as the experimental instructions. Existing work provides little guidance on modeling these complex relationships, and many ad hoc assumptions would be needed. ${ }^{13}$

A limitation of our paper is that the compromise-effect parameter values we estimate are specific to our experimental setting, and thus cannot be extrapolated to other settings (see related points in Levitt and List 2007). For example, our experiment includes 64 MPL's, which may induce fatigue among experimental participants, potentially explaining why the compromise effect strengthens from Part A to Part B. However, the methodology we demonstrate-jointly estimating the compromise effect and preference parameters - is general and can be applied and extended in at least three useful directions.

First, the compromise-effect controls that we propose here can be used not only to improve the robustness of estimates of risk preference parameters, but also of parameter estimates for any other preferences elicited using MPLs, including time and other-regarding preferences. Second, our method can be applied to other settings where the compromise effect may play a role, such as Choi, Fisman, Gale, and Kariv's (2007) graphical interface for eliciting preferences, Andreoni and Sprenger's (2012) convex time budget procedure for eliciting time preferences, or consumer choices in the kinds of settings that originally motivated the psychology and marketing research on the compromise effect (Simonson 1989). Finally, the same econometric procedure we implement here- estimating a discrete-choice model that includes additional parameters that capture location in the choice set - could also be applied to measure and control for other types of context effects, such as a tendency to choose items that happen to come at the beginning of a list of alternatives (e.g., as in election ballots; e.g., Koppell and Steen 2004).

\footnotetext{
${ }^{13}$ Sprenger (2015) assumes that the fixed prospect in each binary choice pins down a participant's reference point. Because the fixed prospect was held constant across our scale manipulations, this approach can't explain the effects we find.
} 


\section{REFERENCES}

Abdellaoui, Mohammed, Han Bleichrodt, and Corina Paraschiv. 2007. "Loss Aversion Under Prospect Theory: A Parameter-Free Measurement." Management Science, 53(10): 16591674.

Andersen, Steffen, Glenn W. Harrison, Morten I. Lau, and Elisabet E. Rutström. 2006. "Elicitation Using Multiple Price List Formats." Experimental Economics, 9(4): 383-405.

Andersen, Steffen, Glenn W. Harrison, Morten I. Lau, and Elisabet E. Rutström. 2008. "Eliciting Risk and Time Preferences." Econometrica, 76(3): 583-618.

Andersson, Ola, Håkan J. Holm, Jean-Robert Tyran, and Erik Wengström. 2016. "Risk Aversion Relates to Cognitive Ability: Preferences or Noise?" Journal of the European Economic Association, 14(5): 1129-1154.

Andreoni, James, and Charles Sprenger. 2012. "Estimating Time Preferences from Convex Budgets." American Economic Review, 102(7): 3333-3356.

Apesteguia, Jose, and Miguel A. Ballester. 2018. "Monotone Stochastic Choice Models: The Case of Risk and Time Preferences." Journal of Political Economy, 126(1): 74-106.

Beauchamp, Jonathan P., David Cesarini, and Magnus Johannesson. 2017. "The Psychometric and Empirical Properties of Measures of Risk Preferences." Journal of Risk and Uncertainty, 54(3): 203-237.

Benjamin, Daniel J., Sebastian A. Brown, and Jesse M. Shaprio. 2013. "Who is 'Behavioral'? Cognitive Ability and Anomalous Preferences." Journal of the European Economic Association, 11(6): 1231-1255.

Birnbaum, Michael H. 1992. "Violations of Monotonicity and Contextual Effects in ChoiceBased Certainty Equivalents.” Psychological Science, 3(5): 310-314.

Booij, Adam S., Bernard M.S. van Praag, and Gijs van de Kuilen. 2010. "A parametric analysis of prospect theory's functionals for the general population." Theory and Decision, 68: $115-148$.

Bruhin, Adrian, Helga Fehr-Duda, and Thomas Epper. 2010. "Risk and Rationality: Uncovering Heterogeneity in Probability Distortion.” Econometrica, 78(4): 1375-1412.

Choi, Syngjoo, Raymond Fisman, Douglas Gale, and Shachar Kariv. 2007. "Consistency 
and Heterogeneity of Individual Behavior under Uncertainty." American Economic Review, 97(5): 1921-1938.

Dohmen, Thomas, Armin Falk, David Huffman, and Uwe Sunde. 2010. "Are Risk Aversion and Impatience Related to Cognitive Ability?" American Economic Review, 100(3), 123860.

Dohmen, Thomas, Armin Falk, David Huffman, and Uwe Sunde. 2018. "On the Relationship between Cognitive Ability and Risk Preference." Journal of Economic Perspectives, 32(2): 115-34.

Fehr-Duda, Helga and Thomas Epper. 2012. "Probability and Risk: Foundations and Economic Implications of Probability-Dependent Risk Preferences." Annual Review of Economics, 4:567-593.

Fox, Craig R., and Russell A. Poldrack. 2014. "Prospect Theory and the Brain." In Neuroeconomics: Decision Making and the Brain (Second Edition), ed. P. W. Glimcher and Ernst Fehr, 533-567. Academic Press.

Gächter, Simon, Eric J. Johnson, and Andreas Herrmann. 2007. "Individual-Level Loss Aversion in Riskless and Risky Choices.” IZA Discussion Paper No. 2961.

Harrison, Glenn W., Morten I. Lau, Elisabet E. Rutström, and Melonie B. Sullivan. 2005. "Eliciting Risk and Time Preferences Using Field Experiments: Some Methodological Issues." In Field experiments in economics (Research in Experimental Economics, Volume 10), ed. J. Carpenter, G. Harrison, and J. List, 125-218. Emerald Group Publishing Limited.

Harrison, Glenn W., Morten I. Lau, and Elisabet E. Rutström. 2007. "Estimating Risk Attitudes in Denmark: a Field Experiment." Scandinavian Journal of Economics, 109(2): 341-368. Harrison, Glenn W., John A. List, and Charles Towe. 2007. "Naturally Occurring Preferences and Exogenous Laboratory Experiments: A Case Study of Risk Aversion." Econometrica, 75(2): 433-458.

Harrison, Glenn W. and Elisabet E. Rutström. 2008. "Risk Aversion in the Laboratory." In Risk Aversion in Experiments (Research in Experimental Economics, Volume 12), ed. J. Cox, G. Harrison, 41-196. Emerald Group Publishing Limited.

Hey, John D., and Chris Orme. 1994. "Investigating Generalizations of Expected Utility Theory Using Experimental Data." Econometrica, 62(6): 1291-1326. 
Hjorth, Katrine, and Mogens Fosgerau. 2011. "Loss Aversion and Individual Characteristics." Environmental and Resource Economics, 49(4): 573-596.

Holt, Charles A., and Susan K. Laury. 2002. "Risk Aversion and Incentive Effects." American Economic Review, 92(5); 1644-1655.

Kahneman, Daniel, and Amos Tversky. 1979. "Prospect Theory: An Analysis of Decision Under Risk." Econometrica, 47(2): 263-291.

Kamenica, Emir. 2008. "Contextual Inference in Markets: On the Informational Content of Product Lines." American Economic Review, 98(5): 2127-2149.

Köbberling, Veronika, and Peter Wakker. 2005. "An index of loss aversion." Journal of Economic Theory, 122: 119-131.

Koppell, Jonathan G. S., and Jennifer A. Steen. 2004. "The Effects of Ballot Position on Election Outcomes." Journal of Politics, 66(1): 267-281.

Köszegi, Botond, and Matthew Rabin. 2006. "A Model of Reference-Dependent Preferences." Quarterly Journal of Economics, 121(4): 1133-1165.

Köszegi, Botond, and Matthew Rabin. 2007. "Reference-Dependent Risk Attitudes." American Economic Review, 97(4): 1047-1073.

Levitt, Steven D., and John A. List. 2007. "What do Laboratory Experiments Measuring Social Preferences Reveal About the Real World?" Journal of Economic Perspectives, 21(2): 153174.

Lichtenstein, Sara, Paul Slovic, and Donald Zink. 1969. "The Effect of Instruction in Expected Value Optimality of Gambling Decisions." Journal of Experimental Psychology, 79(2): $236-240$.

Montgomery, Henry, and Thomas Adelbratt. 1982. "Gambling Decisions and Information About Expected Value." Organizational Behavior and Human Performance, 29: 39-57.

Prelec, Drazen. 1998. "The Probability Weighting Function." Econometrica, 66(3): 497-527.

Saha, Atanu. 1993. "Expo-power utility: A 'flexible'form for absolute and relative risk aversion." American Journal of Agricultural Economics, 75(4): 905-913.

Simonson, Itamar. 1989. "Choice Based on Reasons: The Case of Attraction and Compromise Effects." Journal of Consumer Research, 16(2): 158-174.

Slovic, Paul. 1995. "The Construction of Preference." American Psychologist, 50(5): 364-371. 
Sprenger, Charles. 2015. "An Endowment Effect for Risk: Experimental Tests of Stochastic Reference Points." Journal of Political Economy, 123(6): 1456-1499.

Tversky, Amos, and Daniel Kahneman. 1974. "Judgment under Uncertainty: Heuristics and Biases." Science, 185(4157): 1124-1131.

Tversky, Amos, and Daniel Kahneman. 1992. "Advances in Prospect Theory: Cumulative Representation of Uncertainty." Journal of Risk and uncertainty, 5(4): 297-323.

Wakker, Peter P. 2010. Prospect Theory: For Risk and Ambiguity. Cambridge University Press, Cambridge, UK. 
Table 1. ML Estimates of Selected Parameters in the Model with the Compromise Effect

\begin{tabular}{llll}
\hline \hline & $\begin{array}{c}\text { Parts A-D } \\
\text { Together }\end{array}$ & $\begin{array}{l}\text { Part A (Gain } \\
\text { Domain Only) }\end{array}$ & $\begin{array}{l}\text { Part B (Loss } \\
\text { Domain Only) }\end{array}$ \\
\hline \hline$\gamma, \gamma^{+}, \gamma^{-}$ & $0.242^{* * *}$ & $0.448^{* * *}$ & $-0.106^{* *}$ \\
$\lambda$ & $(0.016)$ & $(0.020)$ & $(0.043)$ \\
& $1.311^{* * *}$ & & \\
$\alpha, \alpha^{+}, \alpha^{-}$ & $(0.034)$ & & \\
& $0.619^{* * *}$ & $0.564^{* * *}$ & $0.690^{* * *}$ \\
$\beta, \beta^{+}, \beta^{-}$ & $(0.015)$ & $(0.015)$ & $(0.022)$ \\
& $1.119^{* * *}$ & $0.858^{* * *}$ & $1.471^{* * *}$ \\
$\pi_{1}$ & $(0.025)$ & $(0.033)$ & $(0.061)$ \\
& $-0.091^{* * *}$ & $-0.134^{* * *}$ & $-0.144^{* * *}$ \\
$\pi_{2}$ & $(0.012)$ & $(0.018)$ & $(0.018)$ \\
& $-0.008^{* * *}$ & 0.002 & $-0.004^{*}$ \\
Log-likelihood & $(0.001)$ & $(0.002)$ & $(0.002)$ \\
Wald test for $\pi_{1}, \pi_{2}$ & $p<1 \times 10^{-10}$ & $-23,915$ & $-25,400$ \\
Parameters & 19 & 10 & $p<1 \times 10^{-10}$ \\
Individuals & 493 & 493 & 10 \\
Observations & 30,566 & 13,804 & 493 \\
\hline \hline
\end{tabular}

NOTE: Standard errors are clustered by participant. The log-likelihood statistic is for the model without clustering. The Wald test is for the joint significance of $\pi_{1}$ and $\pi_{2}$.

${ }^{*}$ significant at $10 \%$ level; ${ }^{* *}$ significant at $5 \%$ level; ${ }^{* * *}$ significant at $1 \%$ level. These are tests of the null hypothesis that the coefficient is zero. However, with respect to all parameters (except $\pi_{1}$ and $\pi_{2}$ ) the natural null hypothesis is not equality to 0 . For instance, $\lambda$ is the loss aversion parameter, so the hypothesis of local linearity is $\lambda=1$. We reject this restriction: the t-stat is $(1.311-1) / 0.034=9.15$. 
Table 2. ML Estimates of Selected Parameters in the Parameterized Model with the Compromise Effect

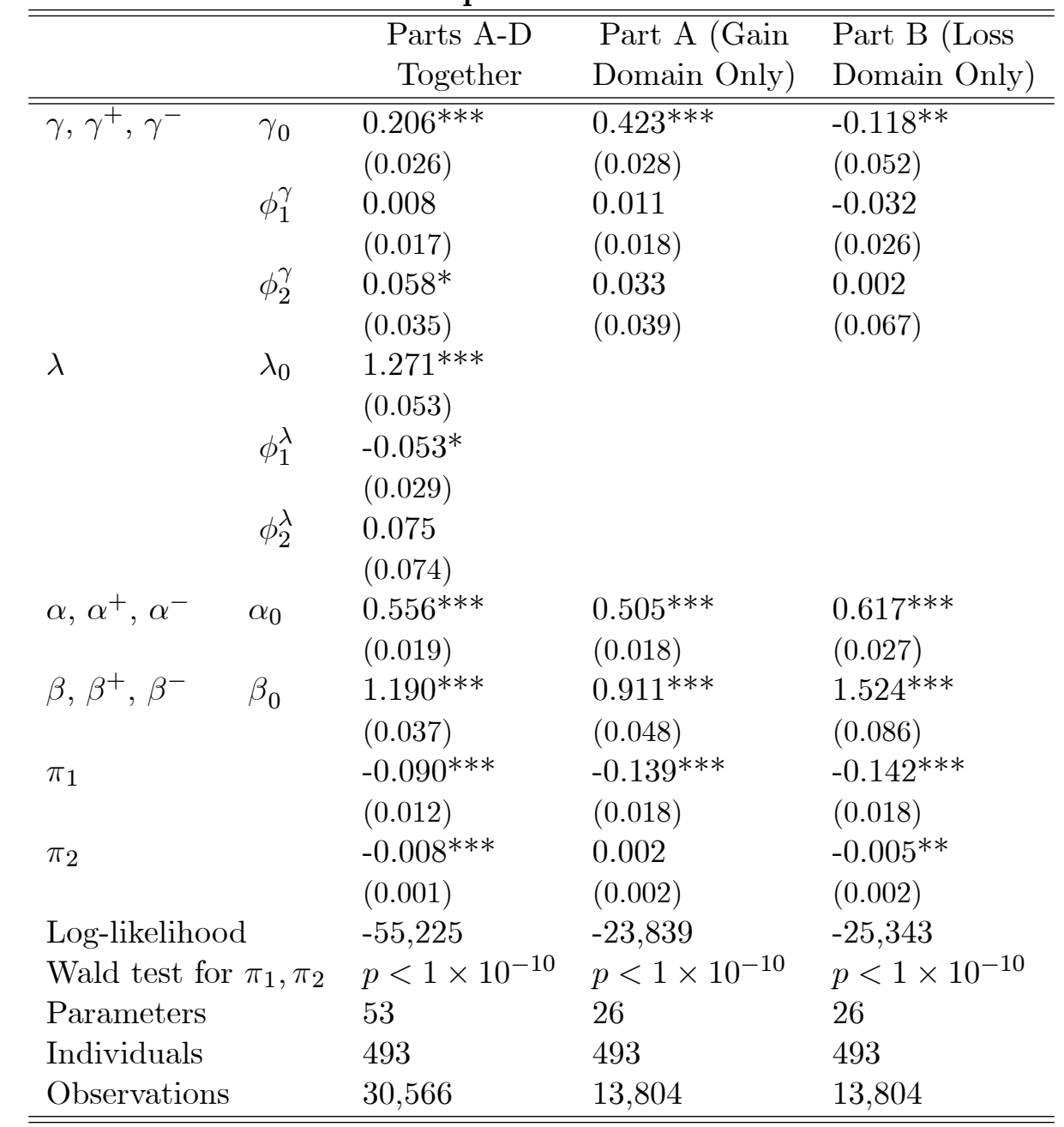

NOTE: Standard errors are clustered by participant. The log-likelihood statistic is for the model without clustering. The Wald test is for the joint significance of $\pi_{1}$ and $\pi_{2}$.

${ }^{*}$ significant at $10 \%$ level; ${ }^{* *}$ significant at $5 \%$ level; ${ }^{* *}$ significant at $1 \%$ level. These are tests of the null hypothesis that the coefficient is zero. 
Table 3. ML Estimates of Selected Parameters in Model Without the Compromise Effect

\begin{tabular}{llll}
\hline \hline & $\begin{array}{c}\text { Parts A-D } \\
\text { Together }\end{array}$ & $\begin{array}{l}\text { Part A (Gain } \\
\text { Domain Only) }\end{array}$ & $\begin{array}{l}\text { Part B (Loss } \\
\text { Domain Only) }\end{array}$ \\
\hline \hline$\gamma, \gamma^{+}, \gamma^{-}$ & $\begin{array}{l}0.203^{* * *} \\
(0.012)\end{array}$ & $0.363^{* * *}$ & -0.010 \\
$\lambda$ & $\begin{array}{l}1.337^{* * *} \\
(0.027)\end{array}$ & & $(0.022)$ \\
& $0.574^{* * *}$ & $0.538^{* * *}$ & \\
$\alpha, \alpha^{+}, \alpha^{-}$ & $(0.010)$ & $(0.011)$ & $0.615^{* * *}$ \\
& $1.123^{* * *}$ & $0.958^{* * *}$ & $(0.013)$ \\
$\beta, \beta^{+}, \beta^{-}$ & $(0.016)$ & $(0.020)$ & $1.296^{* * *}$ \\
& $-59,957$ & $-25,604$ & $-28,141$ \\
Log-likelihood & 8 & 8 \\
Parameters & 17 & 8 & 493 \\
Individuals & 493 & 493 & 13,804 \\
Observations & 30,566 & 13,804 & \\
\hline \hline
\end{tabular}

NOTE: Standard errors are clustered by participant. The log-likelihood statistic is for the model without clustering.

${ }^{*}$ significant at $10 \%$ level; ${ }^{* *}$ significant at $5 \%$ level; ${ }^{* *}$ significant at $1 \%$ level. These are tests of the null hypothesis that the coefficient is zero. 
Table 4. ML Estimates of Selected Parameters in the Parameterized Model Without the Compromise Effect

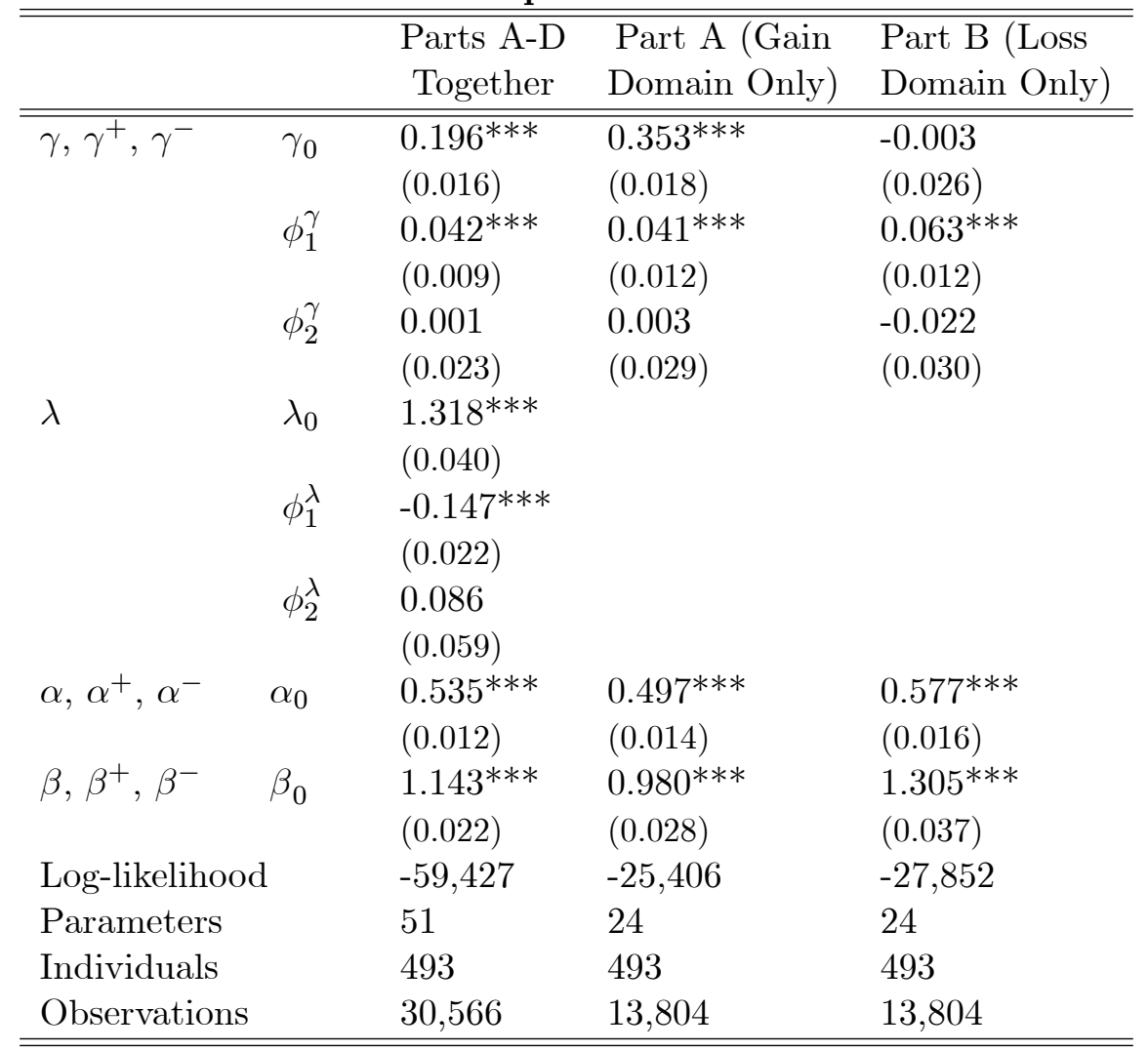

NOTE: Standard errors are clustered by participant. The log-likelihood statistic is for the model without clustering.

${ }^{*}$ significant at $10 \%$ level; ${ }^{* *}$ significant at $5 \%$ level; ${ }^{* * *}$ significant at $1 \%$ level. These are tests of the null hypothesis that the coefficient is zero. 
Table 5. Implied Impact of the Compromise Effect Expressed as a Fraction of the Monetary Value of a Screen's Fixed Prospect $\left(\hat{\theta}_{i}\right)$

\begin{tabular}{ccccc}
\hline \hline & \multicolumn{2}{c}{ Parts A-D Together } & Part A (Gain & Part B (Loss \\
& Prospects from Part A & Prospects from Part B & Domain Only) & Domain only) \\
\hline \hline Row 1 & -0.18 & 0.19 & -0.20 & 0.17 \\
Row 2 & -0.13 & 0.14 & -0.14 & 0.12 \\
Row 3 & -0.08 & 0.08 & -0.07 & 0.06 \\
Row 4 & -0.01 & 0.01 & 0.00 & 0.01 \\
Row 5 & 0.06 & -0.06 & 0.07 & -0.05 \\
Row 6 & 0.14 & -0.13 & 0.14 & -0.12 \\
Row 7 & 0.23 & -0.21 & 0.22 & -0.18 \\
\hline \hline
\end{tabular}

NOTE: As explained in the text, these figures are approximate. 


\section{APPENDIX: Screenshots of the Experiment}

Screenshots of a randomly selected screen from each part of the experiment are shown below for a participant in the Pull -1 and EV treatments. Each scenario appears on a separate screen in the experiment.

This study consists of a total of 64 scenarios, divided into four parts. You have completed 7 of the 64 scenarios.

\section{Part A: Scenario 8 of 28}

For each of questions (a) to $(g)$, please mark your preferred option.

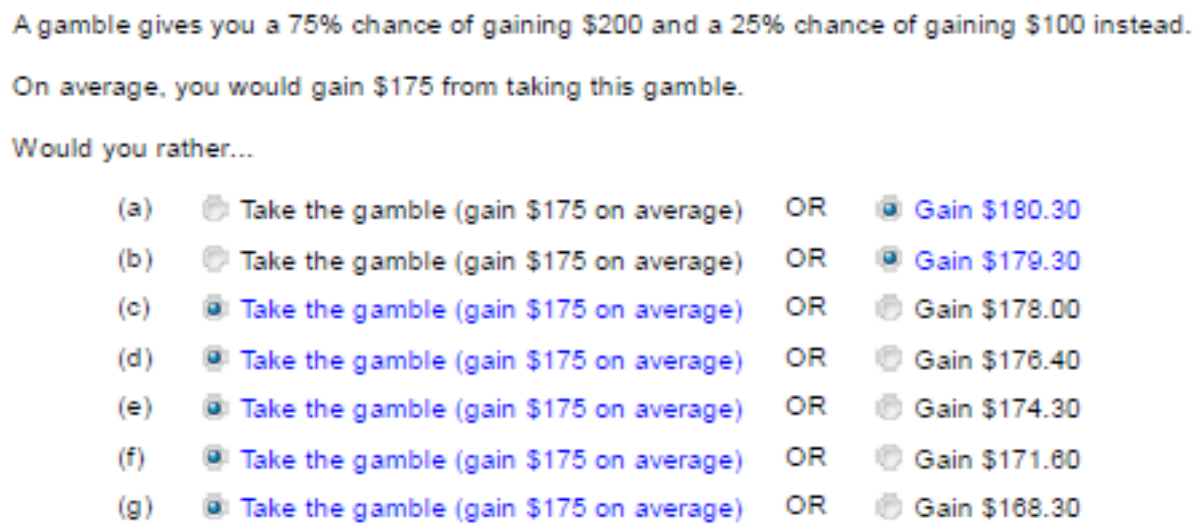

This study consists of a total of 64 scenarios, divided into four parts. You have completed 44 of the 64 scenarios.

\section{Part B: Scenario 17 of 28}

For each of questions (a) to (g), please mark your preferred option.

A gamble gives you a $50 \%$ chance of losing $\$ 150$ and a $50 \%$ chance of losing $\$ 50$ instead.

On average, you would lose $\$ 100$ from taking this gamble.

Would you rather...
(a) Take the gamble (lose $\$ 100$ on average)
OR Lose $\$ 86.70$
(b) Take the gamble (lose $\$ 100$ on average)
OR Lose $\$ 93.80$
(c) Take the gamble (lose $\$ 100$ on average)
OR Lose $\$ 99.30$
(d) Take the gamble (lose $\$ 100$ on average)
OR Lose $\$ 103.70$
(e) Take the gamble (lose $\$ 100$ on average)
OR $\quad$ Lose $\$ 107.10$
(f) Take the gamble (lose $\$ 100$ on average)
OR Lose $\$ 109.70$
(g)
a. Take the gamble (lose $\$ 100$ on average)
OR Lose $\$ 111.80$ 
This study consists of a total of 64 scenarios, divided into four parts. You have completed 56 of the 64 scenarios.

\section{Part C: Scenario 1 of $\mathbf{4}$}

For each of questions (a) to (g). please mark your preferred option.

A gamble gives you a $50 \%$ chance of losing $\$ 50$ and ...

(a) $\quad \ldots$ a $50 \%$ chance of gaining $\$ 0.00$ instead.

(b) ... a $50 \%$ chance of gaining $\$ 42.30$ instead.

(c) ... a $50 \%$ chance of gaining $\$ 75.40$ instead.

(d) ... a $50 \%$ chance of gaining $\$ 101.30$ instead.

(e) ... a $50 \%$ chance of gaining $\$ 121.60$ instead.

(f) $\ldots$ a $50 \%$ chance of gaining $\$ 137.50$ instead.

(g) ... a $50 \%$ chance of gaining $\$ 150.00$ instead.
(9) Take the gamble (lose $\$ 25.00$ on average)

(1) Take the gamble (lose $\$ 3.85$ on average)

(6) Take the gamble (gain $\$ 12.70$ on average)

a. Take the gamble (gain $\$ 25.65$ on average)

a Take the gamble (gain $\$ 35.80$ on average)

a Take the gamble (gain $\$ 43.75$ on average)

a. Take the gamble (gain $\$ 50.00$ on average)
OR Don't take the gamble

OR Don't take the gamble

OR Don't take the gamble

OR Don't take the gamble

OR Don't take the gamble

OR Don't take the gamble

OR Don't take the gamble

This study consists of a total of 64 scenarios, divided into four parts. You have completed 62 of the 64 scenarios.

\section{Part D: Scenario 3 of $\mathbf{4}$}

For each of questions (a) to (g). please mark your preferred option.

Gamble 1 gives you a $50 \%$ chance of losing $\$ 50$ and a $50 \%$ chance of gaining $\$ 150$.

Gamble 2 gives you a $50 \%$ chance of losing $\$ 125$ and ...

(a) $\quad \ldots$ a $50 \%$ chance of gaining $\$ 375.00$ instead.

(b) $\ldots$ a $50 \%$ chance of gaining $\$ 356.30$ instead.

(c) $\ldots$ a $50 \%$ chance of gaining $\$ 332.50$ instead.

(d) ... a $50 \%$ chance of gaining $\$ 302.00$ instead.

(e) ... a $50 \%$ chance of gaining $\$ 263.10$ instead.

(f) $\ldots$ a $50 \%$ chance of gaining $\$ 213.40$ instead.

(g) ... a $50 \%$ chance of gaining $\$ 150.00$ instead. a Take gamble 1 (gain $\$ 50$ on average)

a. Take gamble 1 (gain $\$ 50$ on average)

a. Take gamble 1 (gain $\$ 50$ on average)

- Take gamble 1 (gain $\$ 50$ on average)

a. Take gamble 1 (gain $\$ 50$ on average)

a. Take gamble 1 (gain $\$ 50$ on average)

a Take gamble 1 (gain $\$ 50$ on average)
OR Take gamble 2 (gain $\$ 125.00$ on average)

OR Take gamble 2 (gain $\$ 115.65$ on average)

OR Take gamble 2 (gain $\$ 103.75$ on average)

OR Take gamble 2 (gain $\$ 88.50$ on average)

OR Take gamble 2 (gain $\$ 69.05$ on average)

OR Take gamble 2 (gain $\$ 44.20$ on average)

OR Take gamble 2 (gain $\$ 12.50$ on average) 


\title{
ONLINE APPENDIX
}

\author{
for
}

\section{Controlling for the Compromise Effect Debiases Estimates of Risk Preference Parameters}

\author{
Jonathan P. Beauchamp \\ University of Toronto \\ Daniel J. Benjamin \\ University of Southern California and NBER \\ David I. Laibson \\ Harvard University and NBER \\ Christopher F. Chabris \\ Geisinger Health System
}




\section{Contents}

1 Complete Set of Fixed Prospects and Alternatives for Each Pull Treatment

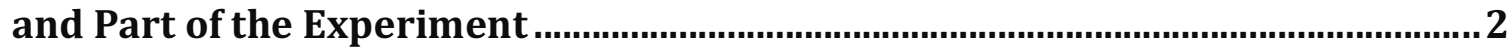

2 Algorithm to Determine the Second Through Sixth Alternatives for Each Pull Treatment and Part of the Experiment ............................................................ 5

3 Summary Statistics of the Raw Data from the Experiment.............................. 6

4 Complete Results for the Estimations Summarized in Tables 1-4 of the

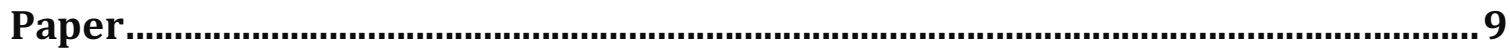

5 Results of Robustness Check with CPT Model with T\&K's Probability Weighting Function............................................................................................. 28

6 Results of Robustness Check with CPT Model with CARA Utility................. 38

7 Results of Robustness Check with CPT Model with Expo-Power Utility.... 48

8 Numerical Estimates of the Parameters for the Compromise Effect $c_{i}$ as a Function of the Row $i$ in Which a Choice Appears 60

9 Additional Information on the Analysis of the Demographic Correlates of the CPT Model and the Compromise Effect Parameters.........................................61

10 Estimates of $\gamma, \gamma^{+}, \gamma^{-}$, and $\lambda$ by EV Treatment in the Models with and

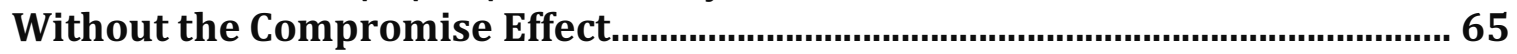

11 Stata Code to Estimate the Baseline CPT Model with the Compromise Effect 67

12 Original Instructions of the Experiment 70

13 References 


\section{Complete Set of Fixed Prospects and Alternatives for Each Pull Treatment and Part of the Experiment}

Below, we list the complete set of fixed prospects and alternative outcomes faced by the participants in the experiment, for each Pull treatment. Online Appendix Table 1.1 lists the fixed prospects and alternative outcomes for Part A (Part B is identical to Part A but with all amounts multiplied by -1). Online Appendix Table 1.2 lists the fixed prospects and the unfixed parts of the alternative prospects for Parts $C$ and D. 


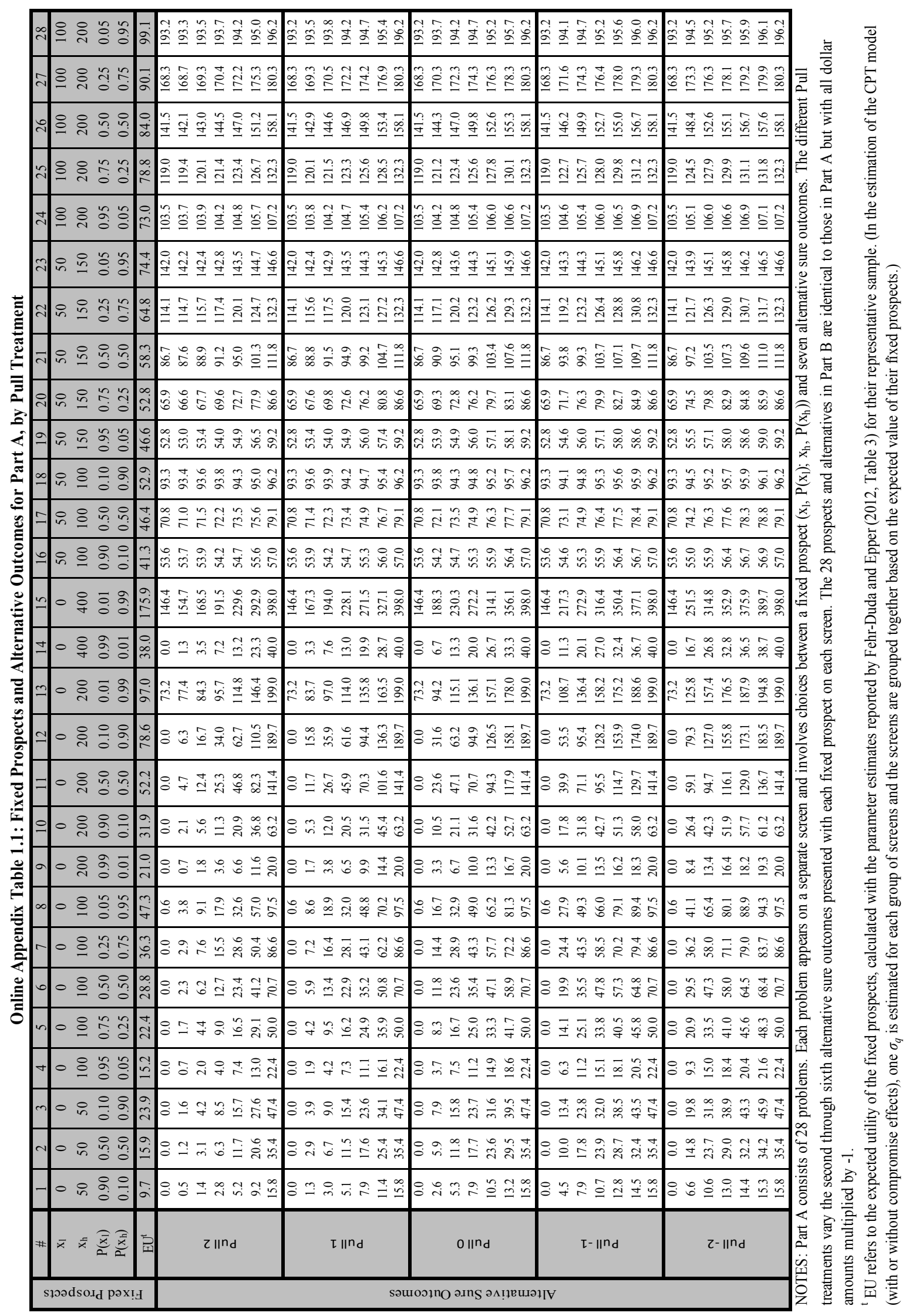


Online Appendix Table 1.2: Fixed Prospects and Unfixed Parts of the Alternative Prospects for Parts C and D, by Pull Treatment

\begin{tabular}{|c|c|c|c|c|c|c|c|c|c|}
\hline & Problem \# & 1 & 2 & 3 & 4 & 5 & 6 & 7 & 8 \\
\hline 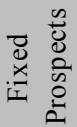 & $\begin{array}{l}\mathrm{x}_{1} \\
\mathrm{x}_{2}\end{array}$ & $\begin{array}{l}0 \\
0\end{array}$ & $\begin{array}{l}0 \\
0\end{array}$ & $\begin{array}{l}0 \\
0\end{array}$ & $\begin{array}{l}0 \\
0\end{array}$ & $\begin{array}{l}-20 \\
50\end{array}$ & $\begin{array}{l}-50 \\
150\end{array}$ & $\begin{array}{c}50 \\
120\end{array}$ & $\begin{array}{l}100 \\
300\end{array}$ \\
\hline \multirow{6}{*}{ 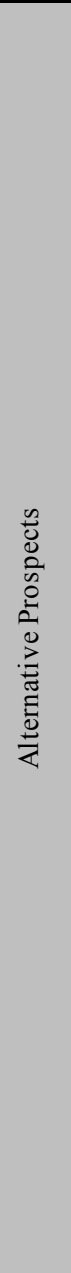 } & $\mathrm{y}_{1}$ & -25 & -50 & -100 & -150 & -50 & -125 & 20 & 25 \\
\hline & \multirow{5}{*}{ 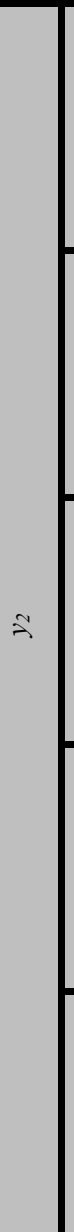 } & $\begin{array}{c}0 \\
2 \\
7 \\
13 \\
25 \\
44 \\
75\end{array}$ & $\begin{array}{c}0 \\
5 \\
13 \\
27 \\
50 \\
87 \\
150\end{array}$ & $\begin{array}{c}0 \\
10 \\
26 \\
54 \\
99 \\
175 \\
300\end{array}$ & $\begin{array}{c}0 \\
15 \\
40 \\
81 \\
149 \\
262 \\
450\end{array}$ & $\begin{array}{c}50 \\
53 \\
58 \\
66 \\
80 \\
102 \\
140\end{array}$ & $\begin{array}{l}150 \\
157 \\
170 \\
190 \\
224 \\
281 \\
375\end{array}$ & $\begin{array}{l}120 \\
123 \\
128 \\
136 \\
150 \\
172 \\
210\end{array}$ & $\begin{array}{l}300 \\
307 \\
320 \\
340 \\
374 \\
431 \\
525\end{array}$ \\
\hline & & $\begin{array}{c}0 \\
6 \\
14 \\
24 \\
37 \\
54 \\
75\end{array}$ & $\begin{array}{c}0 \\
12 \\
28 \\
49 \\
75 \\
108 \\
150\end{array}$ & $\begin{array}{c}0 \\
25 \\
57 \\
97 \\
149 \\
215 \\
300\end{array}$ & $\begin{array}{c}0 \\
37 \\
85 \\
146 \\
224 \\
323 \\
450\end{array}$ & $\begin{array}{c}50 \\
57 \\
67 \\
79 \\
95 \\
115 \\
140\end{array}$ & $\begin{array}{l}150 \\
169 \\
193 \\
223 \\
262 \\
312 \\
375\end{array}$ & $\begin{array}{l}120 \\
127 \\
137 \\
149 \\
165 \\
185 \\
210\end{array}$ & $\begin{array}{l}300 \\
319 \\
343 \\
373 \\
412 \\
462 \\
525\end{array}$ \\
\hline & & $\begin{array}{c}0 \\
13 \\
25 \\
38 \\
50 \\
63 \\
75\end{array}$ & $\begin{array}{c}0 \\
25 \\
50 \\
75 \\
100 \\
125 \\
150\end{array}$ & $\begin{array}{c}0 \\
50 \\
100 \\
150 \\
200 \\
250 \\
300\end{array}$ & $\begin{array}{c}0 \\
75 \\
150 \\
225 \\
300 \\
375 \\
450\end{array}$ & $\begin{array}{c}50 \\
65 \\
80 \\
95 \\
110 \\
125 \\
140\end{array}$ & $\begin{array}{l}150 \\
188 \\
225 \\
263 \\
300 \\
338 \\
375\end{array}$ & $\begin{array}{l}120 \\
135 \\
150 \\
165 \\
180 \\
195 \\
210\end{array}$ & $\begin{array}{l}300 \\
338 \\
375 \\
413 \\
450 \\
488 \\
525\end{array}$ \\
\hline & & $\begin{array}{c}0 \\
21 \\
38 \\
51 \\
61 \\
69 \\
75\end{array}$ & $\begin{array}{c}0 \\
42 \\
75 \\
101 \\
122 \\
138 \\
150\end{array}$ & $\begin{array}{c}0 \\
85 \\
151 \\
203 \\
243 \\
275 \\
300\end{array}$ & $\begin{array}{c}0 \\
127 \\
226 \\
304 \\
365 \\
413 \\
450\end{array}$ & $\begin{array}{c}50 \\
75 \\
95 \\
111 \\
123 \\
133 \\
140\end{array}$ & $\begin{array}{l}150 \\
213 \\
263 \\
302 \\
332 \\
356 \\
375\end{array}$ & $\begin{array}{l}120 \\
145 \\
165 \\
181 \\
193 \\
203 \\
210\end{array}$ & $\begin{array}{l}300 \\
363 \\
413 \\
452 \\
482 \\
506 \\
525\end{array}$ \\
\hline & & $\begin{array}{c}0 \\
31 \\
50 \\
62 \\
68 \\
73 \\
75\end{array}$ & $\begin{array}{c}0 \\
63 \\
100 \\
123 \\
137 \\
145 \\
150\end{array}$ & $\begin{array}{c}0 \\
125 \\
201 \\
246 \\
274 \\
290 \\
300\end{array}$ & $\begin{array}{c}0 \\
188 \\
301 \\
369 \\
410 \\
435 \\
450\end{array}$ & $\begin{array}{c}50 \\
88 \\
110 \\
124 \\
132 \\
137 \\
140\end{array}$ & $\begin{array}{l}150 \\
244 \\
301 \\
335 \\
355 \\
368 \\
375\end{array}$ & $\begin{array}{l}120 \\
158 \\
180 \\
194 \\
202 \\
207 \\
210\end{array}$ & $\begin{array}{l}300 \\
394 \\
451 \\
485 \\
505 \\
518 \\
525\end{array}$ \\
\hline
\end{tabular}

NOTES: Part C consists of Problems 1-4; Part D consists of Problems 5-8. Each problem appears on a separate screen and involves choices between a fixed prospect $\left(\mathrm{x}_{1}, 0.50 ; \mathrm{x}_{2}, 0.50\right)$ and seven alternative prospects $\left(\mathrm{y}_{1}, 0.50 ; \mathrm{y}_{2}, 0.50\right)$. For each problem, $\mathrm{y}_{1}$ is fixed and $\mathrm{y}_{2}$ is unfixed. The different Pull treatments vary the unfixed part $\left(\mathrm{y}_{2}\right)$ of the second through sixth alternative prospects on each screen. 


\section{Algorithm to Determine the Second Through Sixth Alternatives for Each Pull Treatment and Part of the Experiment}

As described in the paper, the Pull 1 and Pull 2 treatments are designed to resemble T\&K's experiment, in which the second through sixth alternatives are "logarithmically spaced between the extreme outcomes of the prospect" (T\&K, p. 305). Conversely, in the Pull -1 and Pull -2 treatments, the alternatives are more densely concentrated at the monetary amounts farther from zero. Pull 2 and Pull -2 are more skewed than Pull 1 and Pull -1.

We use the following algorithm to determine the second through sixth alternative outcomes for screen $q$ in Pull 1 and Pull 2 for Part A (in the gain domain):

- Label the alternative outcomes for screen $q$, in decreasing monetary amounts, $x_{q 1}, x_{q 2}, \ldots, x_{q 7}$ and define $\Delta_{q} \equiv x_{q 1}-x_{q 7}$.

- Recall that (as described in the paper) $x_{q 1}$ and $x_{q 7}$ (the first and seventh alternatives of screen $q$ ) are identical across treatments and correspond to the screen's fixed prospect's certainty equivalents for CRRA expected-utility-maximizers with CRRA parameters $\gamma=-1$ and $\gamma=0.99$.

- For Pull 1, let $k=0.3$ and solve $(1+a)^{6} k \Delta_{q}=(1+k) \Delta_{q}$ for $a$. Then, let $z_{i}=(1+a)^{(7-i)} k \Delta_{q}$, $i=1, \ldots, 7$. These seven $z_{i}$ points form a $\log$ scale from $k \Delta_{q}$ to $(1+k) \Delta_{q}$.

- We then "shift" the log scale formed by these $z_{i}$ points so that the scale starts at $x_{q 7}$ and ends at $x_{q 1}: x_{q i}=z_{i}+\left(x_{q 7}-k \Delta_{q}\right), i=2, \ldots, 6$, and round to the nearest dime.

- The algorithm for Pull 2 is identical, except that we let $k=0.05$.

In Pull -1 and Pull -2 , the spacing between $x_{q i}$ and $x_{q(i+1)}$ is equal to the spacing between $x_{q(7-i)}$ and $x_{q(7-i+1)}(i=1, \ldots, 6)$ in Pull 1 and Pull 2, respectively.

The amounts for Part B are identical to the amounts for Part A, multiplied by -1 .

For Parts C and D, we use the same algorithm to determine the parts of the second through sixth alternatives that are not fixed. (Recall that the alternatives in Parts C and D are risky prospects with two possible realizations, and that one of these two realizations is fixed across the seven alternatives and the other varies across alternatives-i.e. it is not fixed.) 


\section{Summary Statistics of the Raw Data from the Experiment}

Online Appendix Figures 3.1-3.4 show the percentage of choices where the safe option was chosen, by Pull and EV treatments, separately for Parts A, B, C, and D of the experiment. (For Part D, Online Appendix Figure 3.4 shows the percentage of choices where the option involving the smallest possible loss was selected.) The figures also show $p$ values for $t$-tests of the equality of the percentages of safe choices across the two EV treatments within each Pull treatment. To compute the percentages, each row of the MPL was counted as a choice, and data from the 28 participants whose data were excluded from the estimation data for the main analyses in the main text (see Section 3.3 of the main text) were excluded here too. The figure captions provide additional details.

Although these figures may give readers a sense of the underlying data we collected in our experiment, caution is warranted when interpreting them because the different Pull treatments involve different sets of choices, and the raw data are thus not directly comparable across treatments. For example, consider Online Appendix Figure 3.1, which shows the percentages of safe choices in Part A. The percentages are lower in the Pull 2 treatment and higher in the Pull -2 treatment. Recall that the alternative prospects in the Pull 2 treatment involve amounts that are closer to zero, and the alternative prospects in the Pull -2 treatment involve amounts that are further away from zero. In the absence of a compromise effect, a participant with a given certainty equivalent for a gamble on a given screen will thus select the safe option less frequently in the Pull 2 treatment than in the Pull -2 treatment. The existence of a compromise effect would partially mitigate this tendency but would not fully counter it. Because of this, Online Appendix Figure 3.1 shows that the percentage of safe choices decreases in Pull, even though theoretical considerations suggest (see Section 4 of the main text), and our empirical results confirm, that estimates of risk aversion (i.e., $\hat{\gamma}, \hat{\gamma}^{+}, \hat{\gamma}^{-}$) increase in Pull. 


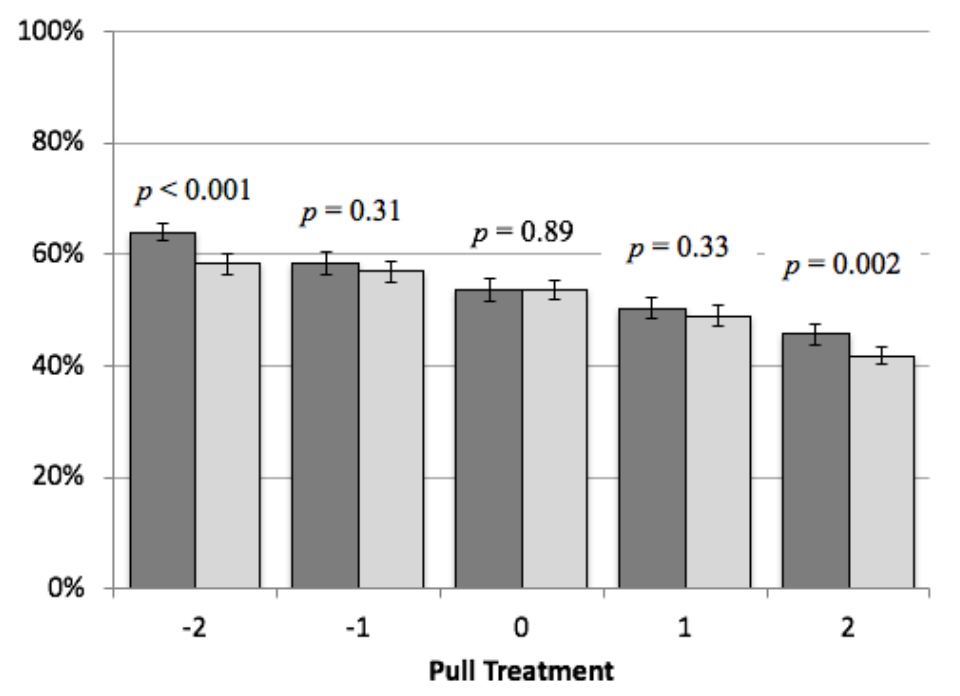

No EV treatment $\square$ EV treatment $\mapsto 95 \%$ Confidence Interval

Online Appendix Figure 3.1. Percentage of choices where the safe option was chosen in Part A, by Pull and EV treatments. (In Part A, the safe options are the alternative prospects; each row of the MPL is counted as a choice.) The $p$ values at the top of the bars are for $t$-tests of the equality of the percentages of safe choices across the two EV treatments within each Pull treatment.

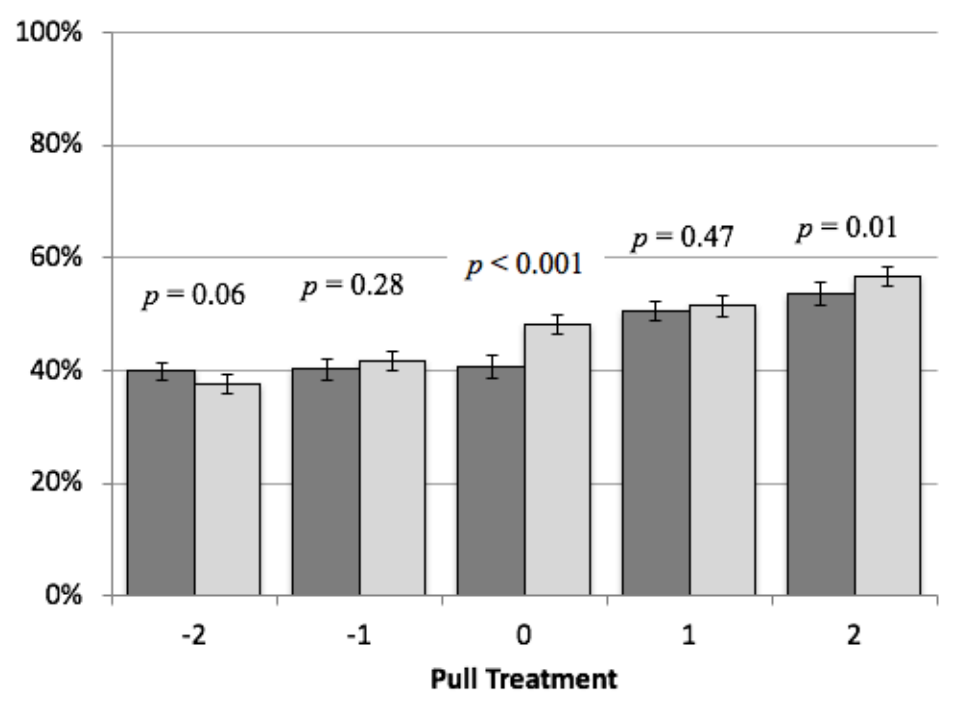

$\square$ No EV treatment $\square$ EV treatment $\mapsto 95 \%$ Confidence Interval

Online Appendix Figure 3.2. Percentage of choices where the safe option was chosen in Part B, by Pull and EV treatments. (In Part B, the safe options are the alternative prospects; each row of the MPL is counted as a choice.) The $p$ values at the top of the bars are for $t$-tests of the equality of the percentages of safe choices across the two EV treatments within each Pull treatment. 


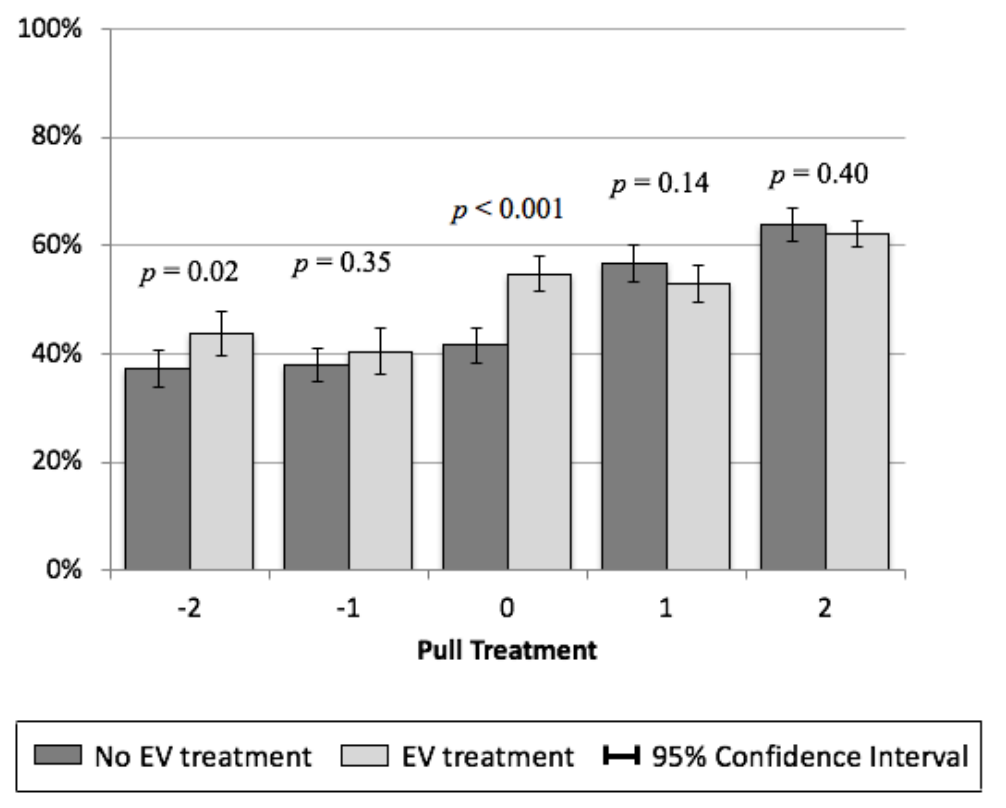

Online Appendix Figure 3.3. Percentage of choices where the safe option was chosen in Part C, by Pull and EV treatments. (In Part C, we define the safe option in a row as selecting "Don't take the gamble"; each row of the MPL is counted as a choice.) The $p$ values at the top of the bars are for $t$-tests of the equality of the percentages of safe choices across the two EV treatments within each Pull treatment.

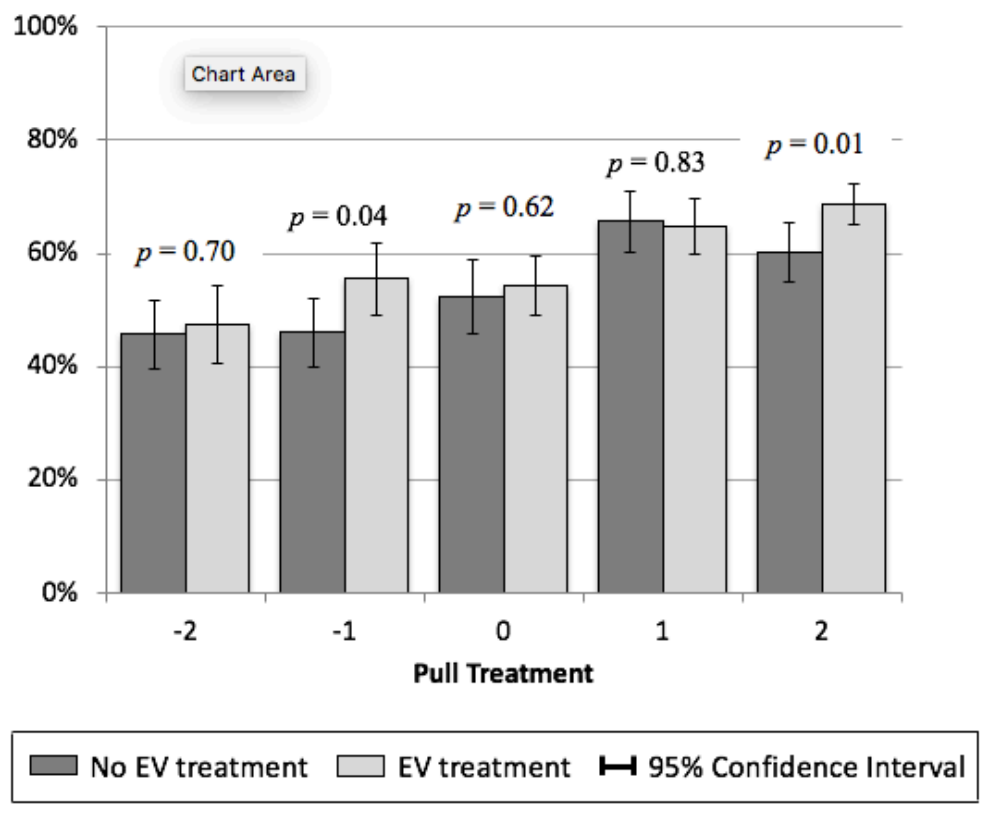

Online Appendix Figure 3.4. Percentage of choices where the option involving the smaller possible loss was chosen in Part D, by Pull and EV treatments. (All choices in Part D involve two gambles, gamble 1 and gamble 2, each of which involves a 50\% chance of a loss; the possible loss in gamble 1 is always smaller than that in gamble 2; thus, the figure shows the percentage of choices where gamble 1 was selected; each row of the MPL is counted as a choice.) The $p$ values at the top of the bars are for $t$-tests of the equality of the percentages of safe choices across the two EV treatments within each Pull treatment. 


\section{Complete Results for the Estimations Summarized in Tables 1-4 of the Paper}




\subsection{Complete Results for Table 1 in the Paper: ML Estimates of All Parameters in the Model with the Compromise Effect}

\subsubsection{Parts A-D Together}

\begin{tabular}{|c|c|c|c|}
\hline & $\begin{array}{l}\text { Number of obs } \\
\text { Wald chi2 }(0)\end{array}$ & $\begin{array}{l}= \\
=\end{array}$ & $\begin{array}{r}30566 . \\
.\end{array}$ \\
\hline Log pseudolikelihood $=-55378.806$ & Prob > chi2 & $=$ & \\
\hline
\end{tabular}

(Std. Err. adjusted for 493 clusters in subjectId)






\subsubsection{Part A (Gain Domain Only)}

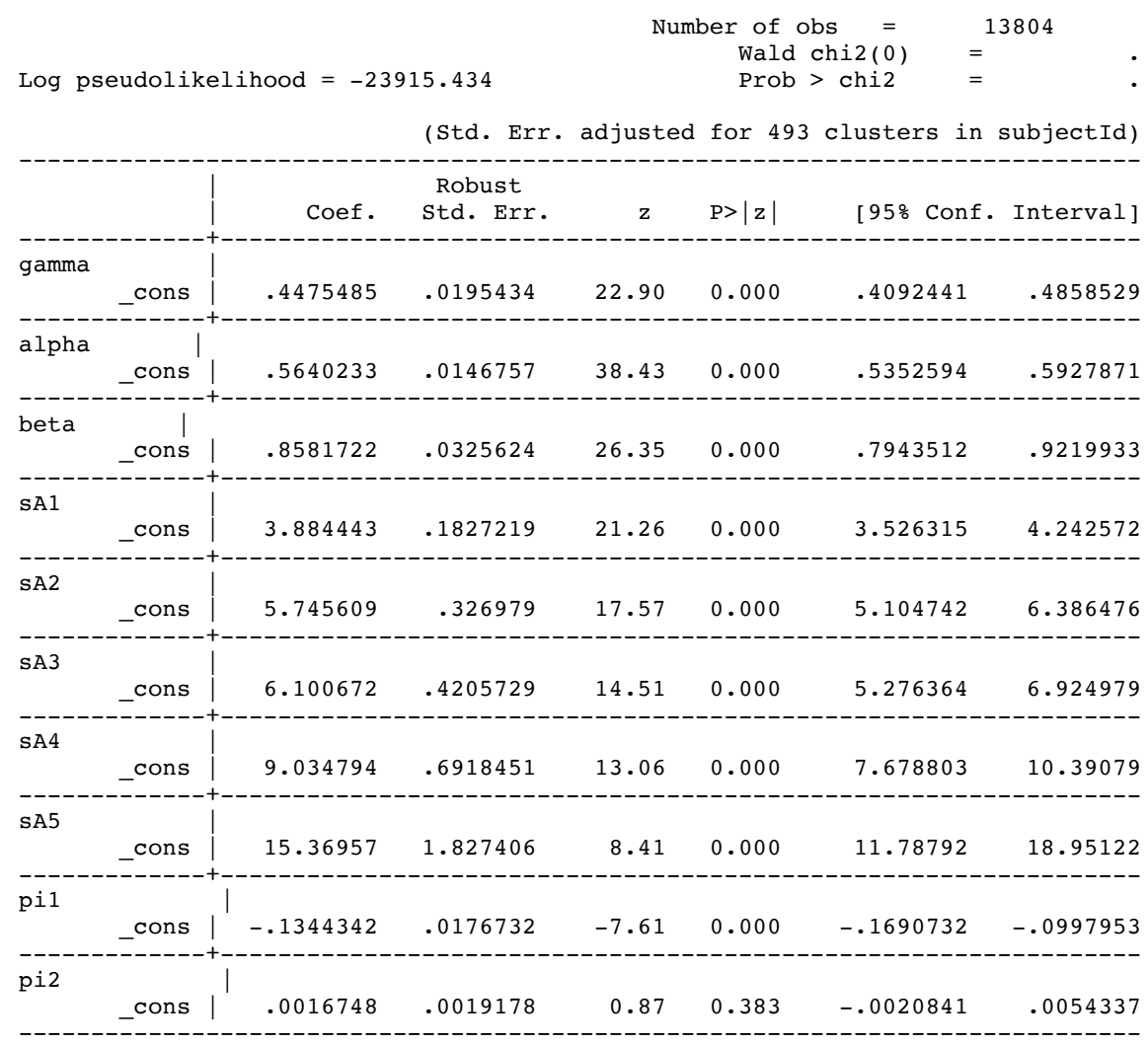




\subsubsection{Part B (Loss Domain Only)}

\begin{tabular}{|c|c|c|c|c|}
\hline & & $\begin{array}{l}\text { Number of obs } \\
\text { Wald chi2 }(0)\end{array}$ & $\begin{array}{l}= \\
=\end{array}$ & $\begin{array}{r}13804 \\
.\end{array}$ \\
\hline Log pseudolikelihood $=$ & -25399.65 & Prob > chi2 & $=$ & . \\
\hline
\end{tabular}

(Std. Err. adjusted for 493 clusters in subjectId)

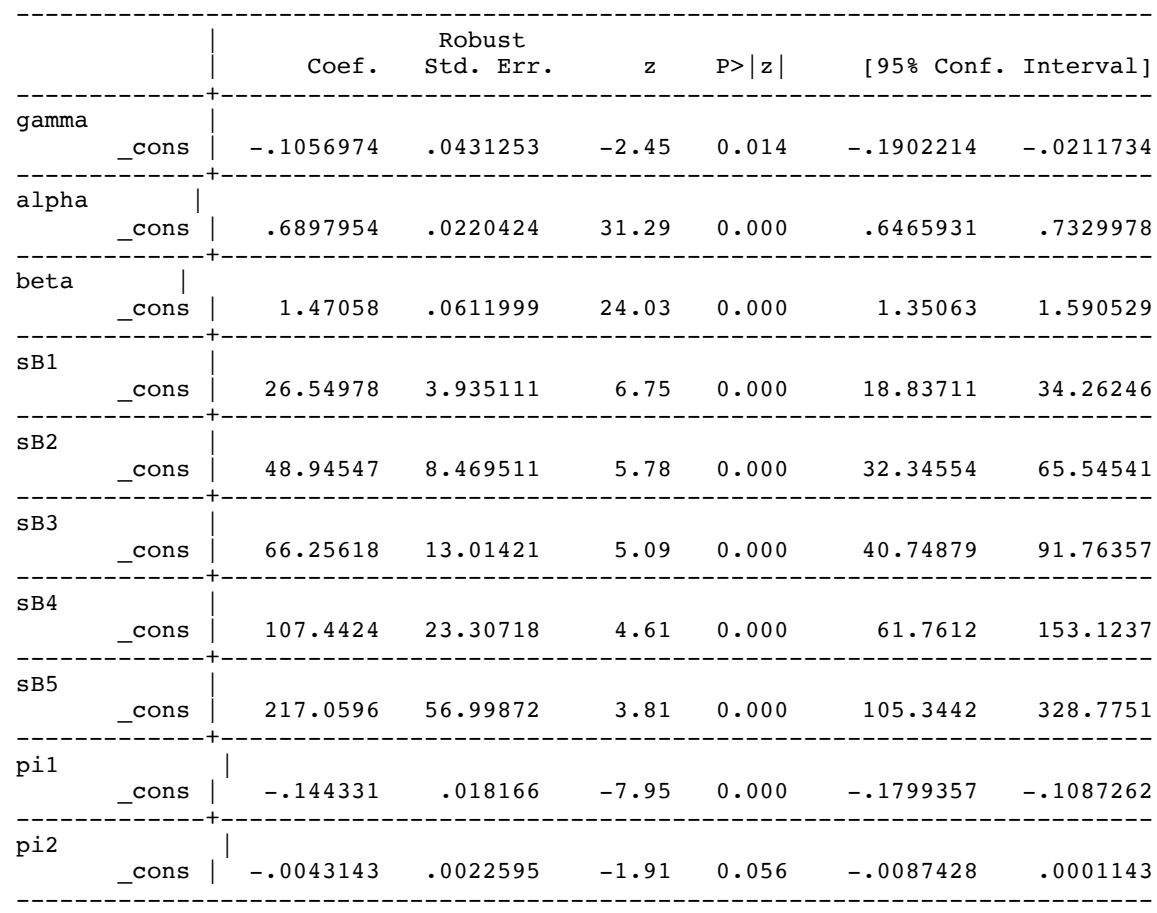




\subsection{Complete Results for Table 2 in the Paper: ML Estimates of All Parameters in the Parameterized Model with the Compromise Effect}

\subsubsection{Parts A-D Together}

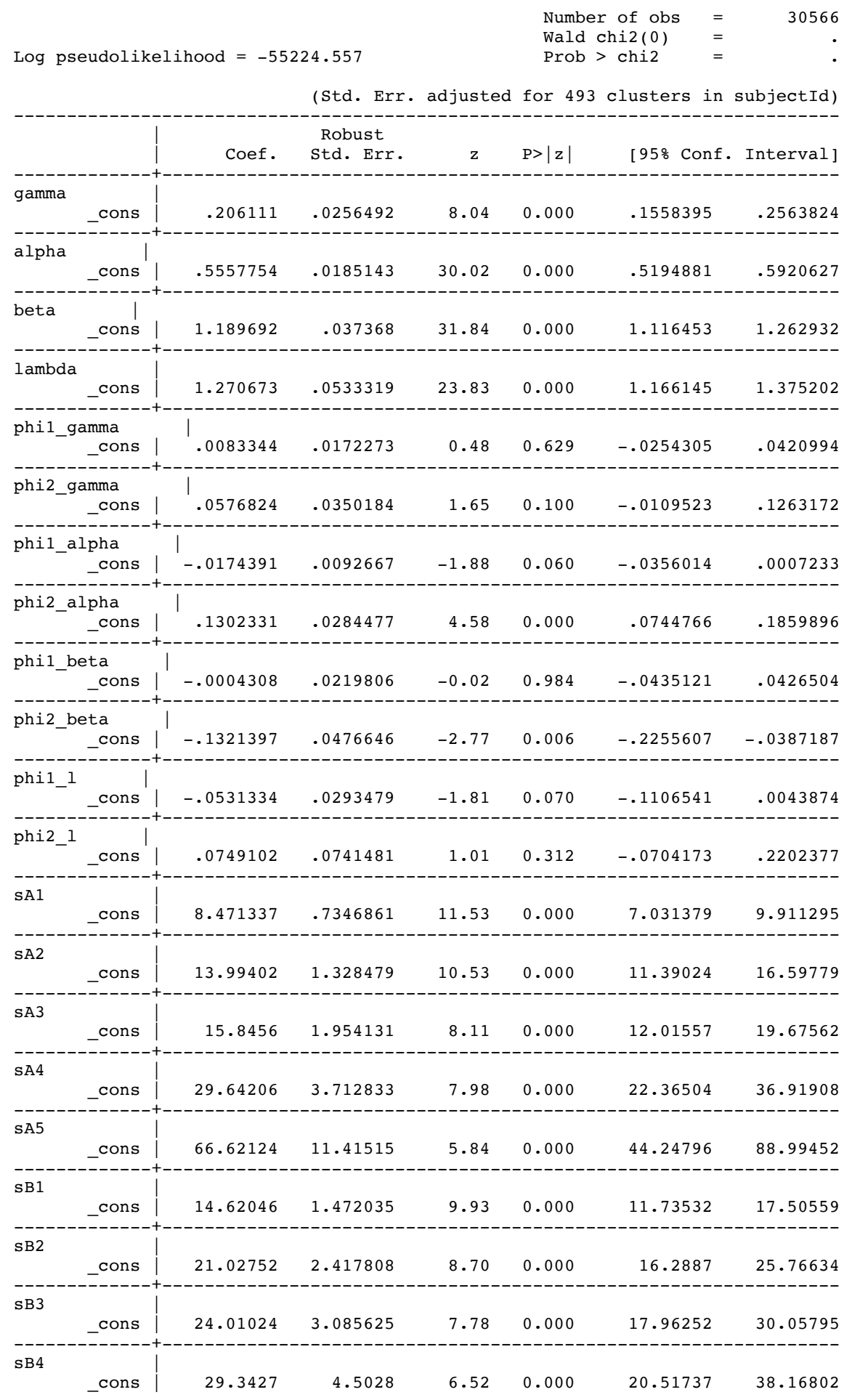




\begin{tabular}{|c|c|c|c|c|c|c|}
\hline C1 & & & & & & \\
\hline _con & 8.091364 & .9009637 & 8.98 & 0.000 & 6.325508 & 9.85722 \\
\hline $\mathrm{sC} 2$ & & & & & & \\
\hline _con & 20.51948 & 2.878159 & 7.13 & 0.000 & 14.8784 & 26.16057 \\
\hline $\mathrm{sD}$ & & & & & & \\
\hline _con & 16.03147 & 2.278884 & 7.03 & 0.000 & 11.56494 & 20.49801 \\
\hline phi1_sA1 & & & & & & \\
\hline$-\mathrm{CO}$ & .0787081 & .3413576 & 0.23 & 0.818 & -.5903405 & .7477566 \\
\hline phi2_sA1 & & & & & & \\
\hline$-\quad$ cons & -2.757494 & .8594604 & -3.21 & 0.001 & -4.442005 & -1.072982 \\
\hline phi1_sA2 & & & & & & \\
\hline - cons & .0824369 & .7700597 & 0.11 & 0.915 & -1.426852 & 1.591726 \\
\hline phi2_sA2 & $--2------1$ & $-\cdots$ & ----- & $=--$ & --- & (n) \\
\hline- cons & -3.545769 & 1.657203 & -2.14 & 0.032 & -6.793827 & -.2977101 \\
\hline phi1_sA3 & & & & & & \\
\hline$-\quad-\mathrm{CO}$ & .2790316 & 1.095298 & 0.25 & 0.799 & -1.867712 & 2.425776 \\
\hline phi2_sA3 & $--0---2$ & & & & & \\
\hline$-{ }_{-}^{\mathrm{CO}}$ & -1.995147 & 2.573782 & -0.78 & 0.438 & -7.039667 & 3.049372 \\
\hline phil_sA4 & --ー-------- & $=--x_{-1}$ & & & 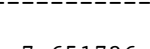 & ( \\
\hline - cons & -3.35317 & 2.193181 & -1.53 & 0.126 & -7.651726 & .9453867 \\
\hline phi2_sA4 & ---------1 & --------- & ------ & ----- & --ー-ー-ー--ー- & -------- \\
\hline -cons & -6.249444 & 4.151368 & -1.51 & 0.132 & -14.38598 & 1.887088 \\
\hline phil_sA5 & & & & & & \\
\hline$-\mathrm{C}$ & -6.356309 & 6.008805 & -1.06 & 0.290 & -18.13335 & 5.420733 \\
\hline phi2_sA5 & & & & & & \\
\hline$-\quad$ cons & -21.68732 & 12.76104 & -1.70 & 0.089 & -46.69849 & 3.323855 \\
\hline phi1_sB1 & & & & & 1051206 & 070720 \\
\hline- cons & -.3858308 & .747751 & -0.52 & 0.606 & -1.851396 & 1.079734 \\
\hline phi2_sB1 & & & & & & , \\
\hline _cons & -3.229005 & 1.798479 & -1.80 & 0.073 & -6.75396 & .2959493 \\
\hline phi1_sB2 & & & & & & \\
\hline - cons & -.8176172 & 1.426382 & -0.57 & 0.567 & -3.613275 & 1.978041 \\
\hline phi2_sB2 & & & & & & \\
\hline -cons & -4.1457 & 2.926864 & -1.42 & 0.157 & -9.882248 & 1.590848 \\
\hline phi1_sB3 & & & & & & \\
\hline _cons & -1.020451 & 1.586598 & -0.64 & 0.520 & -4.130126 & 2.089223 \\
\hline phi2_sB3 & & & & & & \\
\hline$-\quad$ cons & -6.994841 & 3.584155 & -1.95 & 0.051 & -14.01966 & .0299745 \\
\hline phil_sB4 & & & & & & \\
\hline$-\quad$ cons & -2.661792 & 2.884826 & -0.92 & 0.356 & -8.315947 & 2.992364 \\
\hline phi2_sB4 & & & & & & \\
\hline - cons & -4.413494 & 5.222461 & -0.85 & 0.398 & -14.64933 & 5.822341 \\
\hline phi1_sB5 & & & & & & \\
\hline - & -8.1278 & 4.800149 & -1.69 & 0.090 & -17.53592 & 1.28032 \\
\hline phi2_sB5 & & & & & & \\
\hline -cons & -7.753461 & 9.176378 & -0.84 & 0.398 & -25.73883 & 10.23191 \\
\hline phi1_sC1 & & & & & & 4007623 \\
\hline Cons & -.5049022 & .4824912 & -1.05 & 0.295 & -1.450567 & .4407631 \\
\hline phi2_sC1 & & & & & & 165 \\
\hline - cons & -.21316 & 1.213742 & -0.18 & 0.861 & -2.592051 & 2.165731 \\
\hline phi1_sC2 & -1.76451 & 1.556722 & -1.13 & 0.257 & -4.81563 & 1.286609 \\
\hline
\end{tabular}




\begin{tabular}{|c|c|c|c|c|c|c|}
\hline _cons & -1.913247 & 3.618202 & -0.53 & 0.597 & -9.004794 & 5.178299 \\
\hline phil_sD_cons & -1.814308 & 1.001584 & -1.81 & 0.070 & -3.777376 & .14876 \\
\hline phi2_sD_cons & -5.404312 & 2.380116 & -2.27 & 0.023 & -10.06925 & -.7393695 \\
\hline _cons & -.0896071 & .0122324 & -7.33 & 0.000 & -.1135822 & -.065632 \\
\hline _cons & -.0076155 & .0013797 & -5.52 & 0.000 & -.0103198 & -.0049113 \\
\hline
\end{tabular}




\subsubsection{Part A (Gain Domain Only)}

\begin{tabular}{|c|c|c|c|c|c|}
\hline \multicolumn{2}{|c|}{ Log pseudolikelihood $=-23838.856$} & & $\begin{array}{l}\text { Wald } \\
\text { Prob }\end{array}$ & $\begin{array}{ll}\operatorname{hi2}(0) & \text { Number } \\
\operatorname{chi2} & =\end{array}$ & of obs \\
\hline & (Std. Er & $8 \dot{\theta}$ & or & sters in & bject Id \\
\hline Coef. & $\begin{array}{l}\text { Robust } \\
\text { Std. Err. }\end{array}$ & $\mathrm{z}$ & $P>|z|$ & [95\% Conf. & nterval \\
\hline amma & .0278263 & 15.22 & 0.000 & .3689151 & .477992 \\
\hline Lpha_cons & .0183603 & 27.51 & 0.000 & .4691622 & .541133 \\
\hline ta $\begin{array}{r}1 \\
\text { cons }\end{array}$ & .0478594 & 19.03 & 0.000 & .8167942 & 1.004399 \\
\hline hil_gamma & .0178499 & 0.62 & 0.537 & -.0239539 & .0460163 \\
\hline hi2_gamma & .0391302 & 0.85 & 0.393 & -.0432496 & .110138 \\
\hline hil_alpha & .0090778 & -1.66 & 0.097 & -.0328434 & .002740 \\
\hline hi2_alpha & .0276327 & 4.49 & 0.000 & .0700477 & .1783657 \\
\hline $\begin{array}{r}\text { hil_beta } \\
\text { co }\end{array}$ & .0267502 & -0.16 & 0.876 & -.0566015 & .0482574 \\
\hline hi2_beta & .0633599 & -1.50 & 0.132 & -.2195372 & .028829 \\
\hline 4.496528 & .2830979 & 15.88 & 0.000 & 3.941666 & 5.0513 \\
\hline 6.404707 & .4869965 & 13.15 & .000 & 5.450211 & 7.35920 \\
\hline cons & .5770657 & 10.82 & 0.000 & 5.11174 & 7.373796 \\
\hline 10.20512 & 1.10953 & 9.20 & 0.000 & 8.03048 & 12.37976 \\
\hline 18.74995 & 3.110022 & 6.03 & 0.000 & 12.65442 & 24.84548 \\
\hline phil_sA1_cons & .16 & .13 & .894 & -.2929508 & .3354776 \\
\hline phi2_sA1_cons & .3552096 & -3.24 & 0.001 & -1.845579 & -.453183 \\
\hline phi1_sA2_cons | & .318068 & 0.08 & 0.933 & -.5968587 & .6499451 \\
\hline phi2_sA2_cons | -1.144326 & .6541053 & -1.75 & 0.080 & -2.426349 & .1376964 \\
\hline $\begin{array}{c}\text { phil_sA3_cons } \\
\text { c }\end{array}$ & .3820786 & 0.69 & 0.492 & -.486055 & 1.011665 \\
\hline phi2_sA3_cons & .8463344 & -0.44 & .660 & -2.03121 & 1.28636 \\
\hline phil_sA4_cons & .7639582 & -1.28 & 0.201 & -2.474951 & .5197105 \\
\hline phi2_sA4_cons | -1.321611 & 1.373165 & -0.96 & 0.336 & -4.012966 & 1.369743 \\
\hline phi1_sA5_cons | -1.934792 & 1.857262 & -1.04 & 0.298 & -5.574958 & 1.705374 \\
\hline
\end{tabular}




\begin{tabular}{|c|c|c|c|c|c|c|}
\hline${ }^{2}$ _SA5 cons & -4.312429 & 3.602988 & -1.20 & 0.231 & -11.37416 & 2.749297 \\
\hline _cons & -.1387867 & .0178033 & -7.80 & 0.000 & -.1736807 & -.1038928 \\
\hline _con & .0023069 & .0018789 & 1.23 & 0.220 & -.0013756 & .0059895 \\
\hline
\end{tabular}




\subsubsection{Part B (Loss Domain Only)}

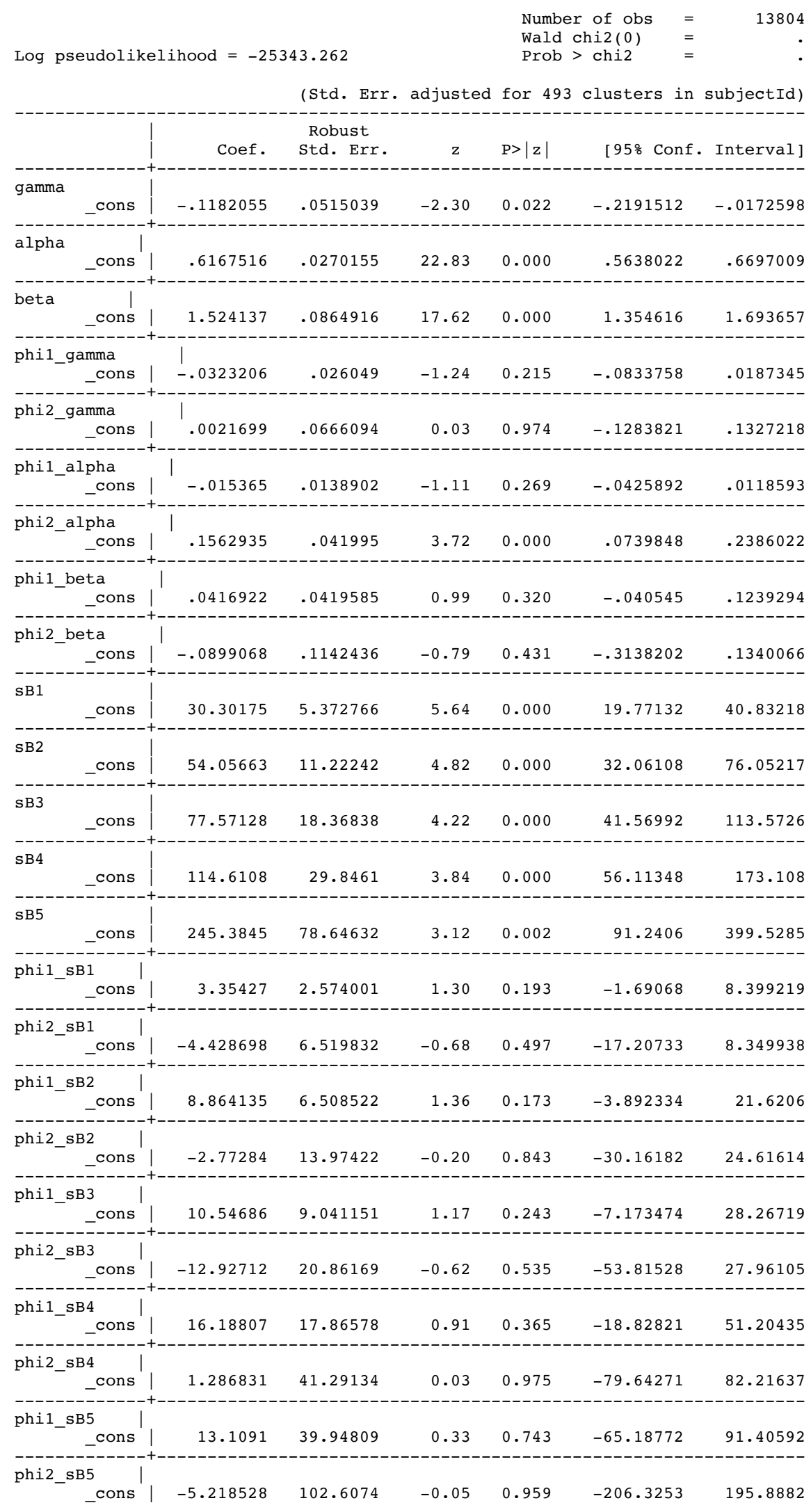




\begin{tabular}{|c|c|c|c|c|c|c|c|}
\hline pil & _cons | & -.1416346 & .0175073 & -8.09 & 0.000 & -.1759483 & -.107321 \\
\hline pi2 & cons & -.0048271 & .0022178 & -2.18 & 0.030 & -.0091739 & -.0004803 \\
\hline
\end{tabular}




\subsection{Complete Results for Table 3 in the Paper: ML Estimates of All Parameters in the Model Without the Compromise Effect}

\subsubsection{Parts A-D Together}

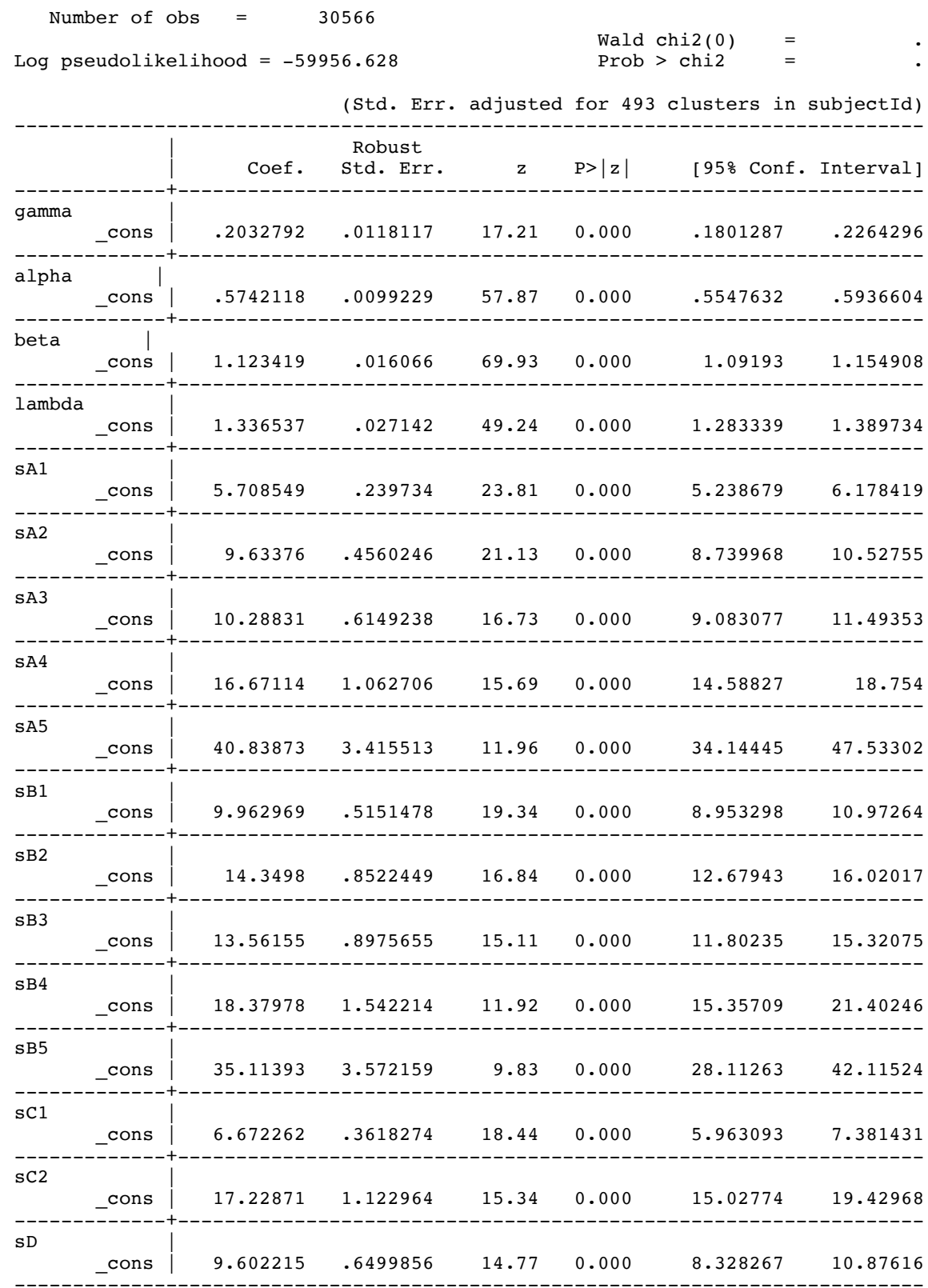




\subsubsection{Part A (Gain Domain Only)}

\begin{tabular}{|c|c|c|c|}
\hline & Number of obs & $=$ & 13804 \\
\hline & Wald chi2(0) & $=$ & . \\
\hline Log pseudolikelihood $=-25604.111$ & Prob > chi2 & $=$ & • \\
\hline
\end{tabular}

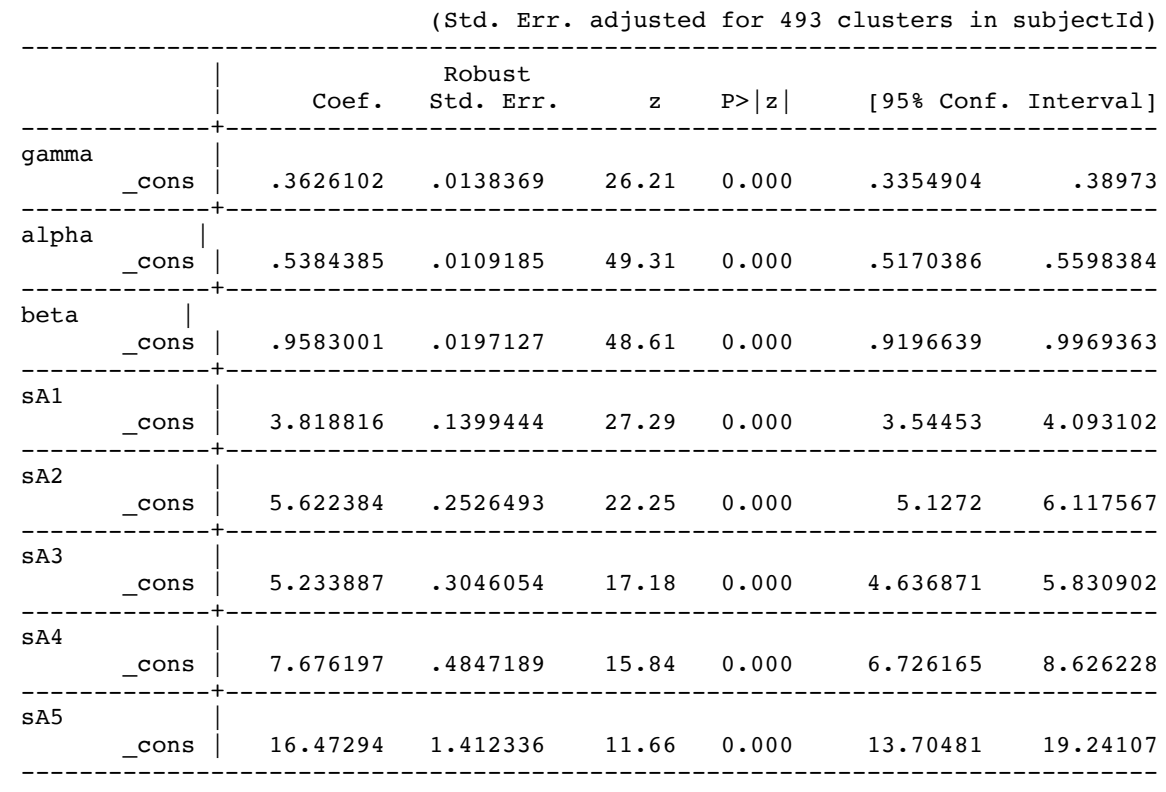




\subsubsection{Part B (Loss Domain Only)}

$\begin{array}{lll}\text { Number of obs } & = & 13804 \\ \text { Wald } \operatorname{chi2}(0) & = & \text { Prob }>\operatorname{chi2}\end{array}=$.

(Std. Err. adjusted for 493 clusters in subjectId)

\begin{tabular}{|c|c|c|c|c|c|c|}
\hline & Coef. & $\begin{array}{c}\text { Robust } \\
\text { Std. Err. }\end{array}$ & $\mathrm{z}$ & $\mathrm{P}>|\mathrm{z}|$ & [95\% Conf. & Interval] \\
\hline \multicolumn{7}{|l|}{ gamma } \\
\hline _cons & -.009619 & .021813 & -0.44 & 0.659 & -.0523716 & .0331337 \\
\hline \multicolumn{7}{|l|}{ alpha } \\
\hline _cons & .6153074 & .0130953 & 46.99 & 0.000 & .589641 & .6409737 \\
\hline _cons & 1.296382 & .0301646 & 42.98 & 0.000 & 1.23726 & 1.355503 \\
\hline sB1 & & & & & & \\
\hline cons & 12.89018 & .973089 & 13.25 & 0.000 & 10.98297 & 14.7974 \\
\hline sB2 & & & & & & \\
\hline _cons & 22.02498 & 1.999303 & 11.02 & 0.000 & 18.10642 & 25.94354 \\
\hline sB3 & & & & & & \\
\hline _cons & 24.2498 & 2.615078 & 9.27 & 0.000 & 19.12434 & 29.37526 \\
\hline sB4 & & & & & & \\
\hline _cons & 38.28514 & 4.688734 & 8.17 & 0.000 & 29.09539 & 47.47489 \\
\hline sB5 & & & & & & \\
\hline _cons & 90.63866 & 13.18273 & 6.88 & 0.000 & 64.80098 & 116.4763 \\
\hline
\end{tabular}




\subsection{Complete Results for Table 4 in the Paper: ML Estimates of All Parameters in the Parameterized Model Without the Compromise Effect}

\subsubsection{Parts A-D Together}

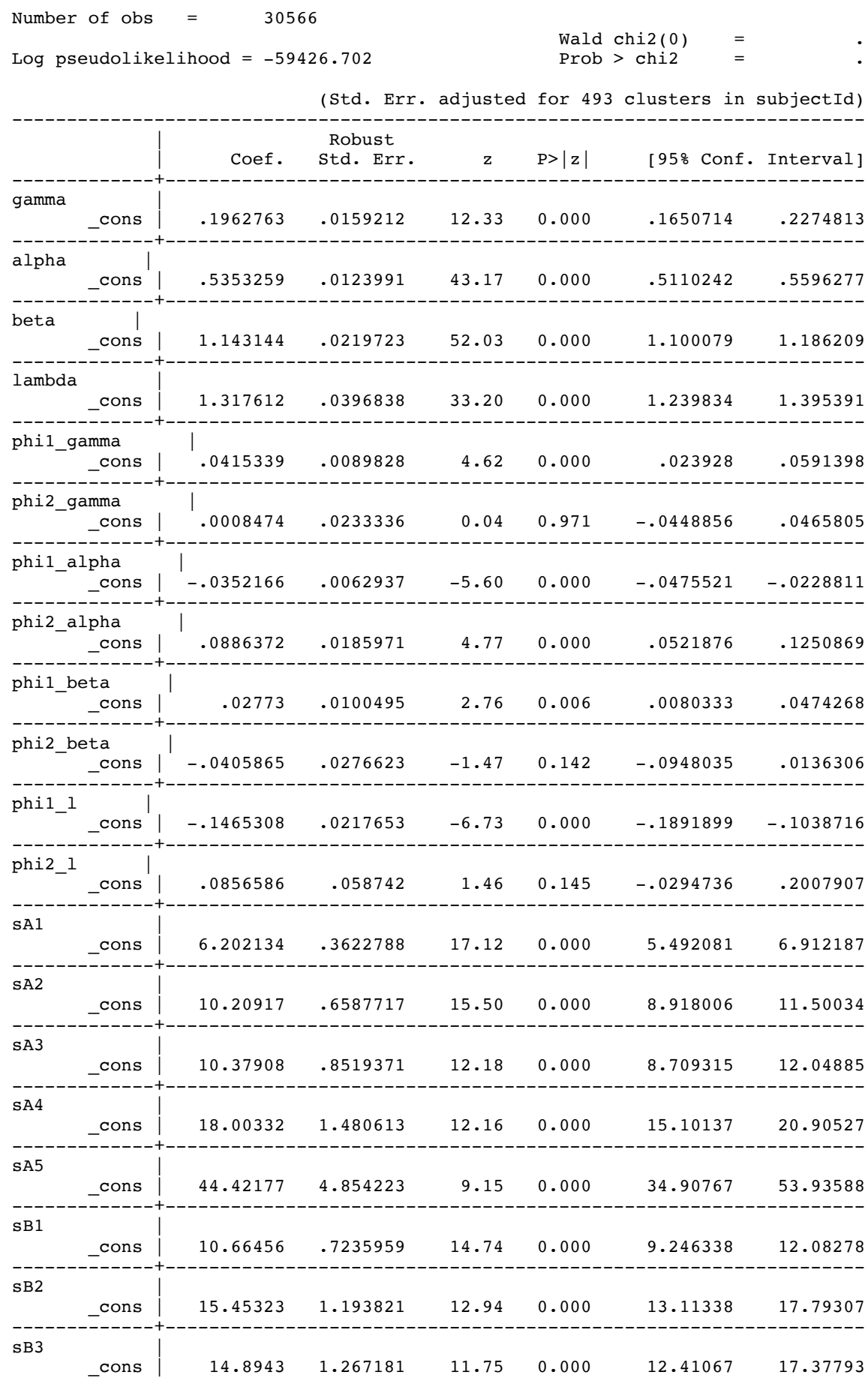




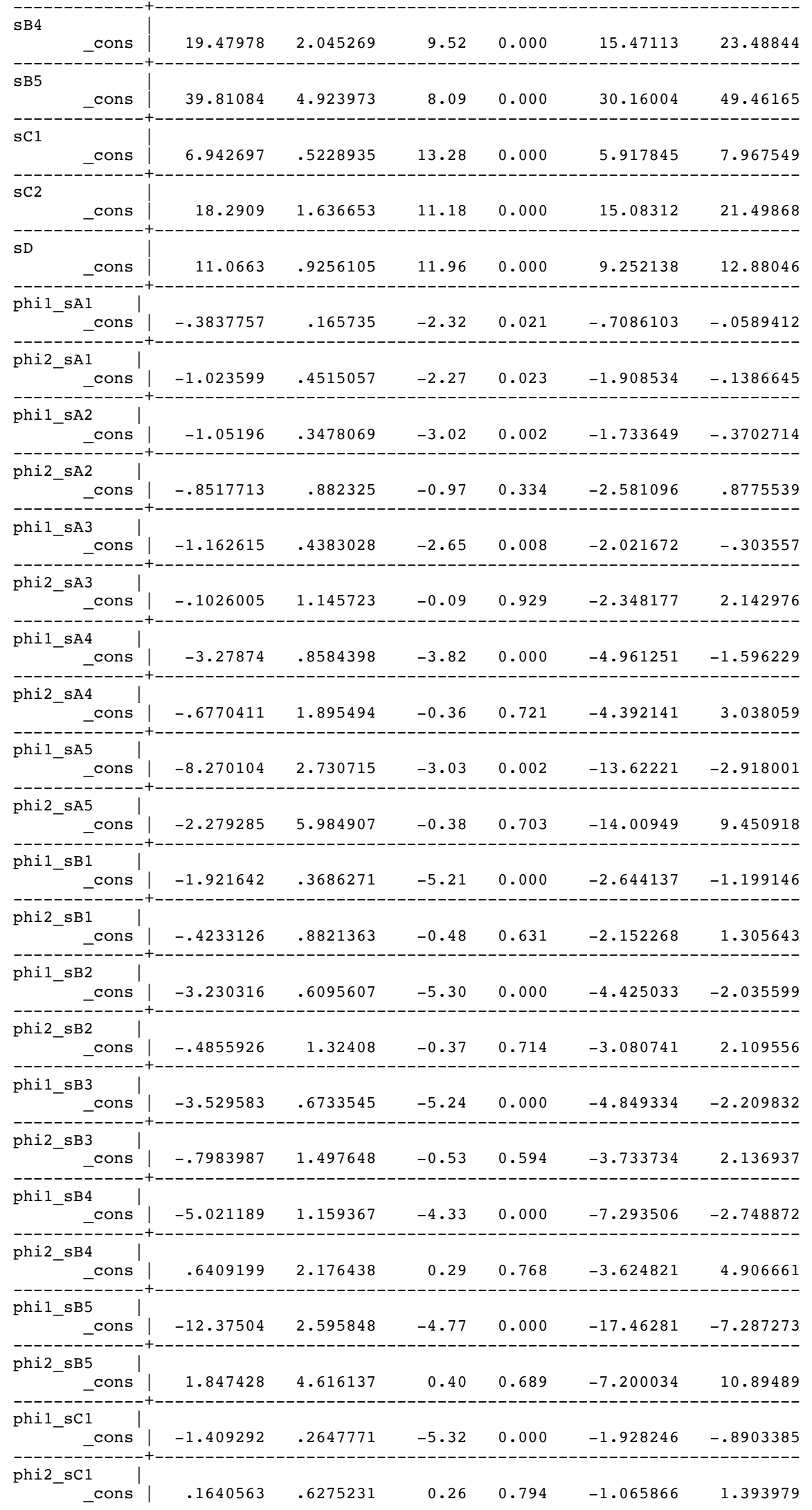




\begin{tabular}{|c|c|c|c|c|c|c|}
\hline _cons & -4.479169 & .8319998 & -5.38 & 0.000 & -6.109859 & -2.848479 \\
\hline phi2_sC2_cons & .2207494 & 1.770406 & 0.12 & 0.901 & -3.249184 & 3.690682 \\
\hline${ }^{\text {phil_sD_cons }}$ & -2.736951 & .5005148 & -5.47 & 0.000 & -3.717942 & -1.75596 \\
\hline phi2_sD_cons & -.8269326 & 1.058469 & -0.78 & 0.435 & -2.901493 & 1.247628 \\
\hline
\end{tabular}




\subsubsection{Part A (Gain Domain Only)}

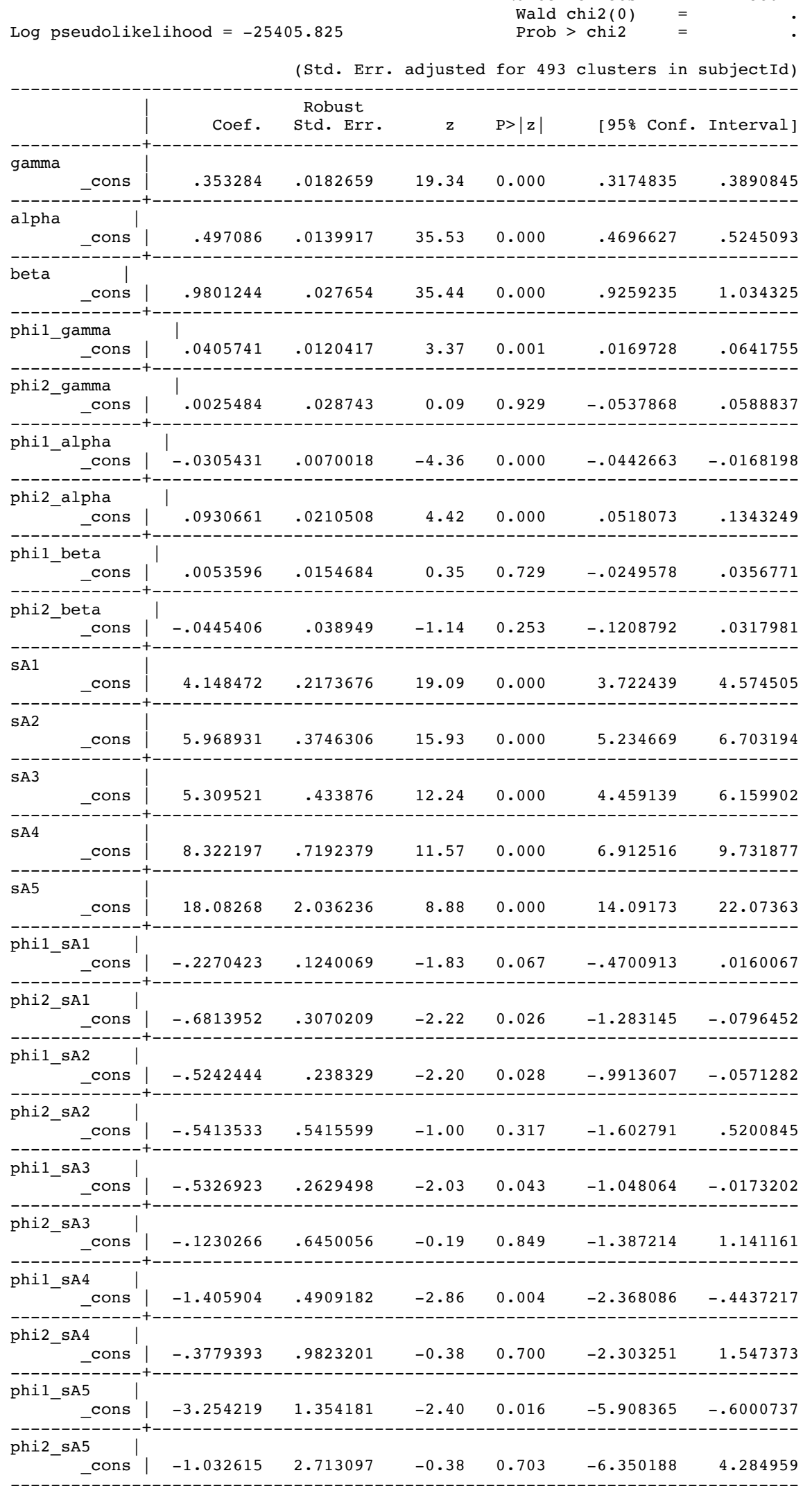




\subsubsection{Part B (Loss Domain Only)}

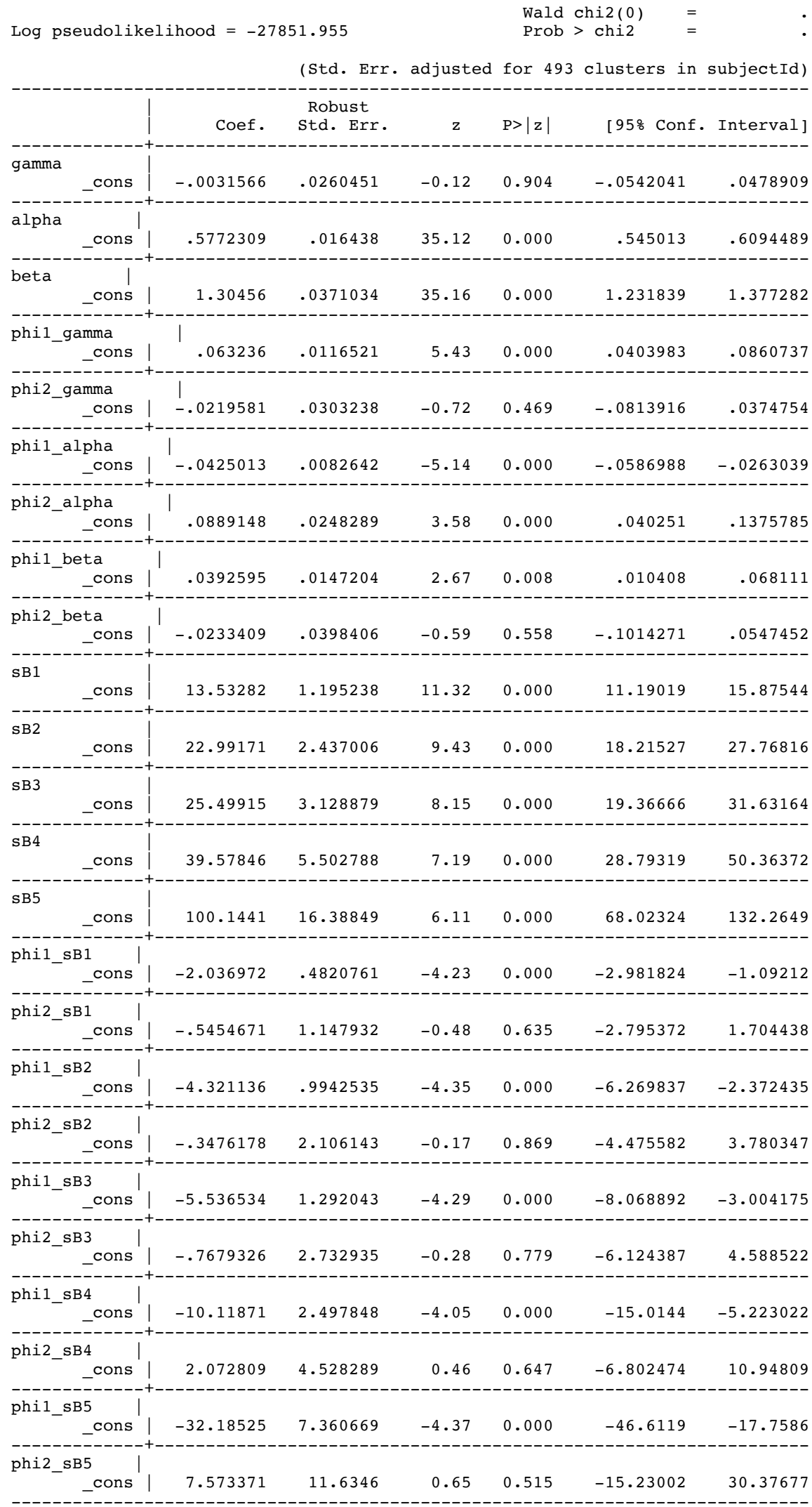




\section{Results of Robustness Check with CPT Model with T\&K's Probability Weighting Function}

As a robustness check, we estimated the CPT model with T\&K's probability weighting function $\left(\omega(p)=p^{\alpha} /\left(p^{\alpha}+(1-p)^{\alpha}\right)^{1 / \alpha}\right)$ instead of the Prelec (1998) probability weighting function. As in the baseline model, utility $u(\cdot)$ is assumed to take the CRRA form (a.k.a. "power utility"), $u(\cdot)=\frac{x^{1-\gamma}}{1-\gamma}$.

Online Appendix Figures 7.1-7.5 and Online Appendix Tables 7.1-7.4 below are analogous to Figures 2-6 and Tables 1-4 in the main text, respectively, except that the results were obtained by estimating the CPT model with T\&K's probability weighting function. 


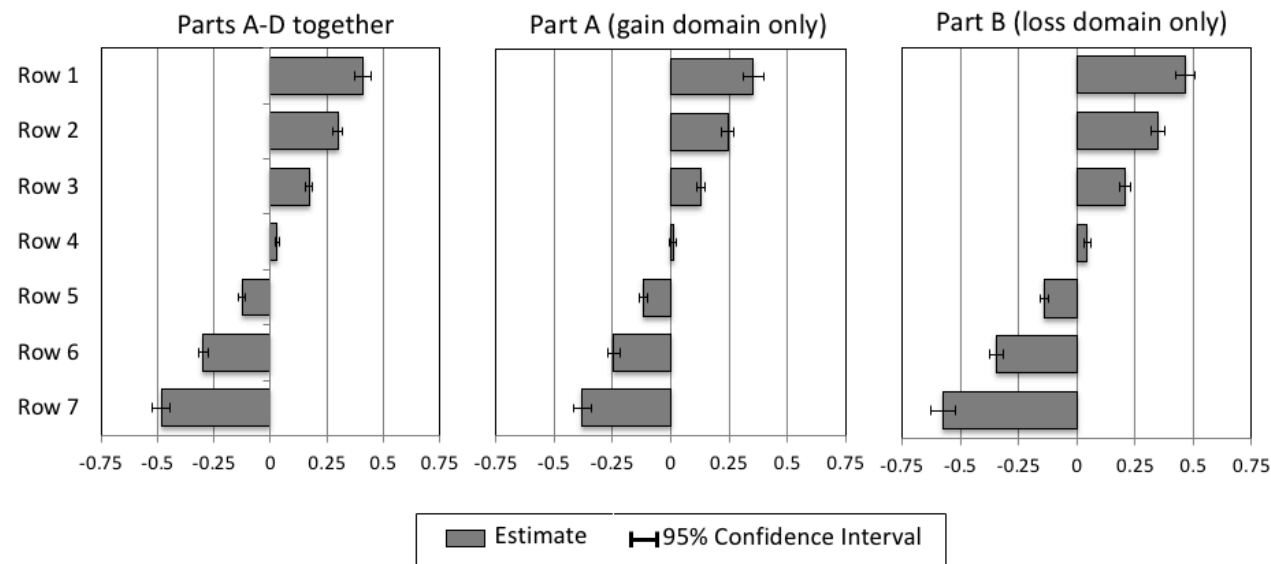

Online Appendix Figure 5.1. Implied estimates of the parameters for the compromise effect $c_{i}$ as a function of the row $i$ in which a choice appears. This figure is analogous to Figure 2 in the main text, except that the results were obtained by estimating the CPT model with T\&K's probability weighting function. 

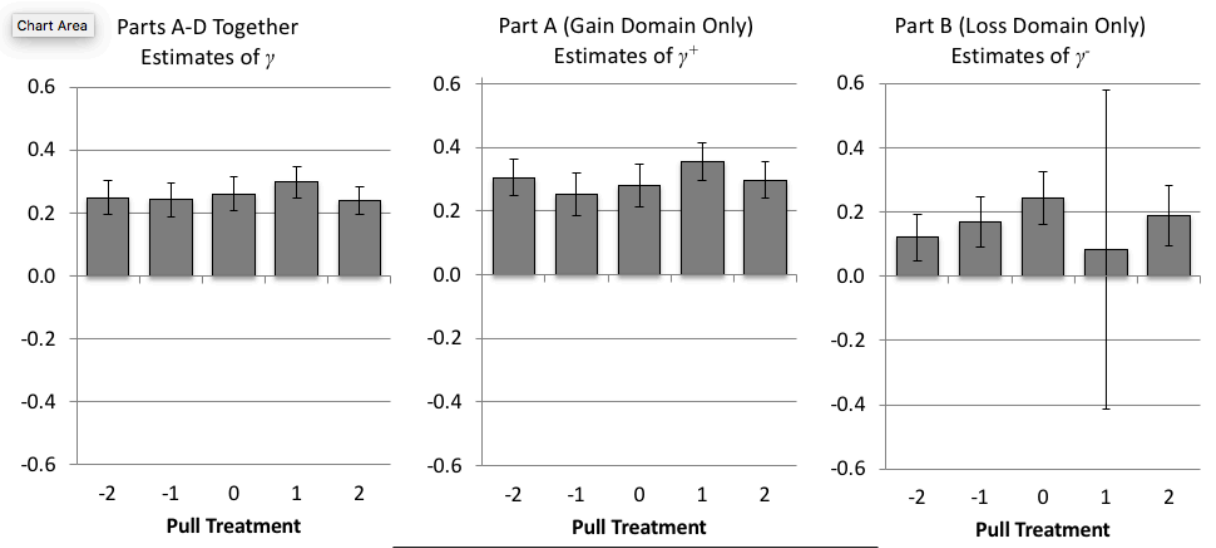

$\square$ Estimate $\quad \mapsto 95 \%$ Confidence Interval

Online Appendix Figure 5.2. Estimates of $\gamma, \gamma^{+}$and $\gamma^{-}$by Pull treatment, from the model with the compromise effect. This figure is analogous to Figure 3 in the main text, except that the results were obtained by estimating the CPT model with T\&K's probability weighting function. 


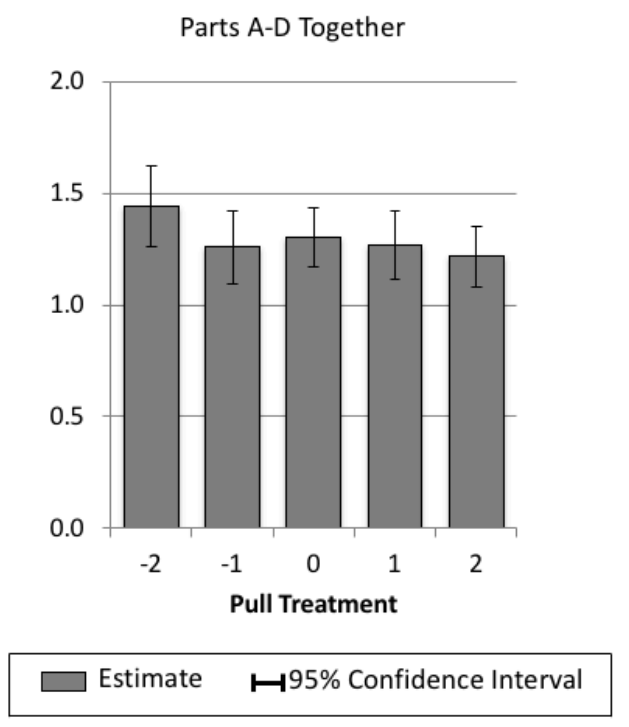

Online Appendix Figure 5.3. Estimates of $\lambda$ by Pull treatment from the model with the compromise effect, for Parts A-D together. This figure is analogous to Figure 4 in the main text, except that the results were obtained by estimating the CPT model with T\&K's probability weighting function. 

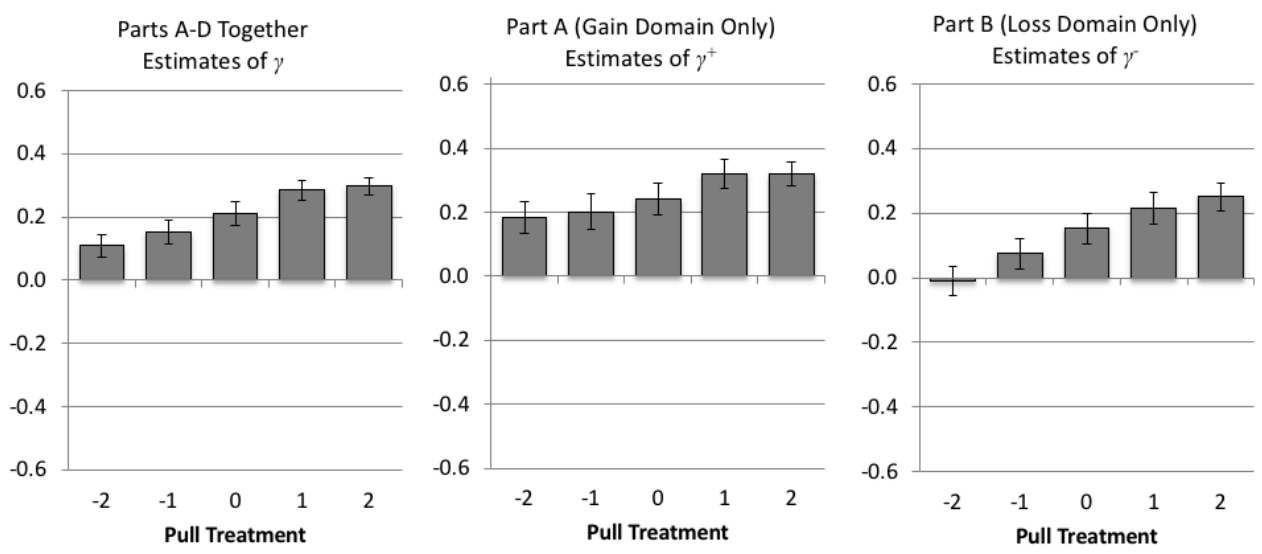

$\square$ Estimate $\quad$ $195 \%$ Confidence Interval

Online Appendix Figure 5.4. Estimates of $\gamma, \gamma^{+}$and $\gamma^{-}$by Pull treatment, from the model without the compromise effect. This figure is analogous to Figure 5 in the main text, except that the results were obtained by estimating the CPT model with T\&K's probability weighting function. 


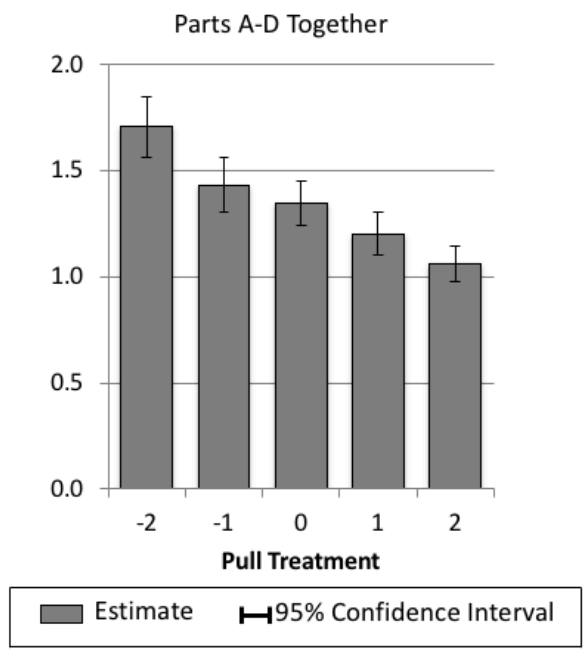

Online Appendix Figure 5.5. Estimates of $\lambda$ by Pull treatment from the model without the compromise effect, for Parts A-D together. This figure is analogous to Figure 6 in the main text, except that the results were obtained by estimating the CPT model with T\&K's probability weighting function. 


\section{Online Appendix Table 5.1. ML Estimates of Selected Parameters in the Model with}

the Compromise Effect

\begin{tabular}{|c|c|c|c|}
\hline & $\begin{array}{l}\text { Parts A-D } \\
\text { Together } \\
\end{array}$ & $\begin{array}{l}\text { Part A (Gain } \\
\text { Domain Only) }\end{array}$ & $\begin{array}{l}\text { Part B (Loss } \\
\text { Domain Only) }\end{array}$ \\
\hline$\gamma, \gamma^{+}, \gamma^{-}$ & $\begin{array}{l}0.267 * * * \\
(0.011)\end{array}$ & $\begin{array}{l}0.298 * * * \\
(0.014)\end{array}$ & $\begin{array}{l}0.202 * * * \\
(0.018)\end{array}$ \\
\hline$\lambda$ & $\begin{array}{l}1.292 * * * \\
(0.034)\end{array}$ & & \\
\hline$\alpha, \alpha^{+}, \alpha^{-}$ & $\begin{array}{l}0.645^{* * *} \\
(0.011)\end{array}$ & $\begin{array}{l}0.617 * * * \\
(0.011)\end{array}$ & $\begin{array}{l}0.689 * * * \\
(0.019)\end{array}$ \\
\hline$\pi_{1}$ & $\begin{array}{l}-0.089 * * * \\
(0.012)\end{array}$ & $\begin{array}{l}-0.102 * * * \\
(0.017)\end{array}$ & $\begin{array}{l}-0.084 * * * \\
(.017)\end{array}$ \\
\hline$\pi_{2}$ & $\begin{array}{l}-0.008 * * * \\
(0.001)\end{array}$ & $\begin{array}{l}-0.003 \\
(0.002)\end{array}$ & $\begin{array}{l}-0.011 * * * \\
(0.002)\end{array}$ \\
\hline Log-likelihood & $-55,357$ & $-24,018$ & $-25,537$ \\
\hline Wald test for $\pi_{1}, \pi_{2}$ & $p<1 \times 10^{-144}$ & $p<1 \times 10^{-80}$ & $p<1 \times 10^{-122}$ \\
\hline Parameters & 18 & 9 & 9 \\
\hline Individuals & 493 & 493 & 493 \\
\hline Observations & 30,566 & 13,804 & 13,804 \\
\hline
\end{tabular}

NOTE: Standard errors are clustered by participant. The log-likelihood statistic is for the model without clustering. The Wald test is for the joint significance of $\pi_{1}$ and $\pi_{2}$. This table is analogous to Table 1 in the main text, except that the results were obtained by estimating the CPT model with T\&K's probability weighting function.

$*$ significant at $10 \%$ level; $* *$ significant at $5 \%$ level; *** significant at $1 \%$ level. 


\section{Online Appendix Table 5.2. ML Estimates of Selected Parameters in the Parameterized Model with the Compromise Effect}

\begin{tabular}{|c|c|c|c|c|}
\hline & & $\begin{array}{l}\text { Parts A-D } \\
\text { Together }\end{array}$ & $\begin{array}{l}\text { Part A (Gain } \\
\text { Domain Only) }\end{array}$ & $\begin{array}{l}\text { Part B (Loss } \\
\text { Domain Only) }\end{array}$ \\
\hline \multirow[t]{3}{*}{$\gamma, \gamma^{+}, \gamma^{-}$} & $\overline{\gamma_{0}}$ & $\begin{array}{l}0.247 * * * \\
(0.018)\end{array}$ & $\begin{array}{l}0.283 * * * \\
(0.021)\end{array}$ & $\begin{array}{l}0.185 * * * \\
(0.025)\end{array}$ \\
\hline & $\phi_{1}^{\gamma}$ & $\begin{array}{l}0.003 \\
(0.009)\end{array}$ & $\begin{array}{l}0.004 \\
(0.010)\end{array}$ & $\begin{array}{l}0.006 \\
(0.014)\end{array}$ \\
\hline & $\phi_{2}^{\gamma}$ & $\begin{array}{l}0.027 \\
(0.024)\end{array}$ & $\begin{array}{l}.0196 \\
(0.028)\end{array}$ & $\begin{array}{l}0.018 \\
(0.034)\end{array}$ \\
\hline \multirow[t]{3}{*}{$\lambda$} & $\lambda_{0}$ & $\begin{array}{l}1.24 * * * \\
(0.049)\end{array}$ & & \\
\hline & $\phi_{1}^{\lambda}$ & $\begin{array}{l}-0.048^{*} \\
(0.025)\end{array}$ & & \\
\hline & $\phi_{2}^{\lambda}$ & $\begin{array}{l}0.094 \\
(0.069)\end{array}$ & & \\
\hline$\alpha, \alpha^{+}, \alpha^{-}$ & & $\begin{array}{l}0.597 * * * \\
(0.014)\end{array}$ & $\begin{array}{l}0.577 * * * \\
(.014)\end{array}$ & $\begin{array}{l}0.630 * * * \\
(0.021)\end{array}$ \\
\hline$\pi_{1}$ & & $\begin{array}{l}-0.088 * * * \\
(0.012)\end{array}$ & $\begin{array}{l}-0.105^{* * *} \\
(0.017)\end{array}$ & $\begin{array}{l}-0.078 * * * \\
(0.017)\end{array}$ \\
\hline$\pi_{2}$ & & $\begin{array}{l}-0.008 * * * \\
(0.001)\end{array}$ & $\begin{array}{l}-0.002 \\
(0.002)\end{array}$ & $\begin{array}{l}-0.012 * * * \\
(0.002)\end{array}$ \\
\hline Log-likelihood & & $-55,203$ & $-23,942$ & $-25,476$ \\
\hline Wald test for $\pi_{1}, \pi_{2}$ & & $p<1 \times 10^{-134}$ & $p<1 \times 10^{-84}$ & $p<1 \times 10^{-112}$ \\
\hline Parameters & & 50 & 23 & 23 \\
\hline Individuals & & 493 & 493 & 493 \\
\hline Observations & & 30,566 & 13,804 & 13,804 \\
\hline
\end{tabular}

NOTE: Standard errors are clustered by participant. The log-likelihood statistic is for the model without clustering. The Wald test is for the joint significance of $\pi_{1}$ and $\pi_{2}$. This table is analogous to Table 2 in the main text, except that the results were obtained by estimating the CPT model with T\&K's probability weighting function.

* significant at $10 \%$ level; ** significant at $5 \%$ level; *** significant at $1 \%$ level. 
Online Appendix Table 5.3. ML Estimates of Selected Parameters in the Model Without the Compromise Effect

\begin{tabular}{clll}
\hline \hline & $\begin{array}{l}\text { Parts A-D } \\
\text { Together }\end{array}$ & $\begin{array}{l}\text { Part A (Gain } \\
\text { Domain Only) }\end{array}$ & $\begin{array}{l}\text { Part B (Loss } \\
\text { Domain Only) }\end{array}$ \\
\hline \hline$\gamma, \gamma^{+}, \gamma^{-}$ & $0.219^{* * *}$ & $0.260^{* * *}$ & $0.144^{* * *}$ \\
& $(0.008)$ & $(0.011)$ & $(0.012)$ \\
$\lambda$ & $1.32^{* * *}$ & & \\
& $(0.027)$ & & \\
$\alpha, \alpha^{+}, \alpha^{-}$ & $0.615^{* * *}$ & $0.599^{* * *}$ & $0.631^{* * *}$ \\
& $(0.007)$ & $(0.008)$ & $(0.010)$ \\
Log-likelihood & $-59,862$ & $-25,681$ & $-28,223$ \\
Parameters & 16 & 7 & 7 \\
Individuals & 493 & 493 & 493 \\
Observations & 30,566 & 13,804 & 13,804 \\
\hline \hline
\end{tabular}

NOTE: Standard errors are clustered by participant. The log-likelihood statistic is for the model without clustering. This table is analogous to Table 3 in the main text, except that the results were obtained by estimating the CPT model with T\&K's probability weighting function.

$*$ significant at $10 \%$ level; $* *$ significant at $5 \%$ level; *** significant at $1 \%$ level. 
Online Appendix Table 5.4. ML Estimates of Selected Parameters in the Parameterized Model Without the Compromise Effect

\begin{tabular}{|c|c|c|c|c|}
\hline & & $\begin{array}{l}\text { Parts A-D } \\
\text { Together }\end{array}$ & $\begin{array}{l}\text { Part A (Gain } \\
\text { Domain Only) }\end{array}$ & $\begin{array}{l}\text { Part B (Loss } \\
\text { Domain Only) }\end{array}$ \\
\hline \multirow[t]{3}{*}{$\bar{\gamma}, \gamma^{+}, \gamma^{-}$} & $\gamma_{0}$ & $\begin{array}{l}0.206^{* * *} \\
(0.012)\end{array}$ & $\begin{array}{l}0.247 * * * \\
(0.015)\end{array}$ & $\begin{array}{l}0.137 * * * \\
(0.017)\end{array}$ \\
\hline & $\phi_{1}^{\gamma}$ & $\begin{array}{l}0.044 * * * \\
(0.006)\end{array}$ & $\begin{array}{l}0.041 * * * \\
(0.007)\end{array}$ & $\begin{array}{l}0.061 * * * \\
(0.009)\end{array}$ \\
\hline & $\phi_{2}^{\gamma}$ & $\begin{array}{l}0.009 \\
(0.018)\end{array}$ & $\begin{array}{l}0.010 \\
(0.021)\end{array}$ & $\begin{array}{l}-0.003 \\
(0.025)\end{array}$ \\
\hline \multirow[t]{3}{*}{$\lambda$} & $\lambda_{0}$ & $\begin{array}{l}1.31 * * * \\
(0.040)\end{array}$ & & \\
\hline & $\phi_{1}^{\lambda}$ & $\begin{array}{l}-0.144 * * * \\
(0.021)\end{array}$ & & \\
\hline & $\phi_{2}^{\lambda}$ & $\begin{array}{l}0.080 \\
(0.057)\end{array}$ & & \\
\hline$\alpha, \alpha^{+}, \alpha^{-}$ & & $\begin{array}{l}0.587 * * * \\
(0.009)\end{array}$ & $\begin{array}{l}0.571 * * * \\
(0.010)\end{array}$ & $\begin{array}{l}0.605 * * * \\
(0.012)\end{array}$ \\
\hline Log-likelihood & & $-59,334$ & $-25,485$ & $-27,937$ \\
\hline Parameters & & 48 & 21 & 21 \\
\hline Individuals & & 493 & 493 & 493 \\
\hline Observations & & 30,566 & 13,804 & 13,804 \\
\hline
\end{tabular}

NOTE: Standard errors are clustered by participant. The log-likelihood statistic is for the model without clustering. This table is analogous to Table 4 in the main text, except that the results were obtained by estimating the CPT model with T\&K's probability weighting function.

$*$ significant at $10 \%$ level; $* *$ significant at $5 \%$ level; *** significant at $1 \%$ level. 


\section{Results of Robustness Check with CPT Model with CARA Utility}

As a robustness check, we estimated the CPT model with CARA (a.k.a. "exponential")

utility (Köbberling and Wakker 2005), $u(x)=\frac{1-e^{-\alpha_{\text {expo }}^{+} x}}{\alpha_{\text {expo }}^{+}}$if $x \geq 0, u(-x)=\frac{1-e^{-\alpha_{\text {expo }}^{-}|x|}}{\alpha_{\text {expo }}^{-}}$if $x<0$, instead of with CRRA utility. As in the baseline model, we used the Prelec (1998) probability weighting function.

For this robustness check with CARA utility, unlike for the baseline CPT model with CRRA utility, we did not impose the assumption that the parameters for the coefficient of (absolute) risk aversion in the gain and in the loss domains are equal to one another (i.e., we did not assume that $\alpha_{\text {expo }}^{+}=\alpha_{\text {expo }}^{-}$). As Wakker (2010, section 9.6) and Köbberling and Wakker (2005) point out, with CRRA utility, for any $\lambda$ there exists a range of $x$ values for which the ratio of disutility from a sure loss of $x$ to utility from a sure gain of $x, \frac{-\lambda u^{-}(-x)}{u^{+}(x)}$, is smaller than 1 , which is the opposite of loss aversion. This issue does not arise with CARA utility, which makes the interpretation of $\lambda$ in the CPT model with CARA utility with $\alpha_{\text {expo }}^{+} \neq \alpha_{\text {expo }}^{-}$less problematic. (A second issue that arises with both CRRA and CARA utility when assuming different risk aversion parameters in the gain and loss domains is that the ratio of disutility from a sure loss of $x$ to utility from a sure gain of $x, \frac{-\lambda u^{-}(-x)}{u^{+}(x)}$, is not uniformly equal to $\lambda$; this issue also arises with the CPT model with CARA utility, thus making the estimates of $\lambda$ we report below in this section more difficult to interpret.)

Online Appendix Figures 8.1-8.5 and Online Appendix Tables 8.1-8.4 below are analogous to Figures 2-6 and Tables 1-4 in the main text, respectively, except that the results were obtained by estimating the CPT model with CARA utility. 


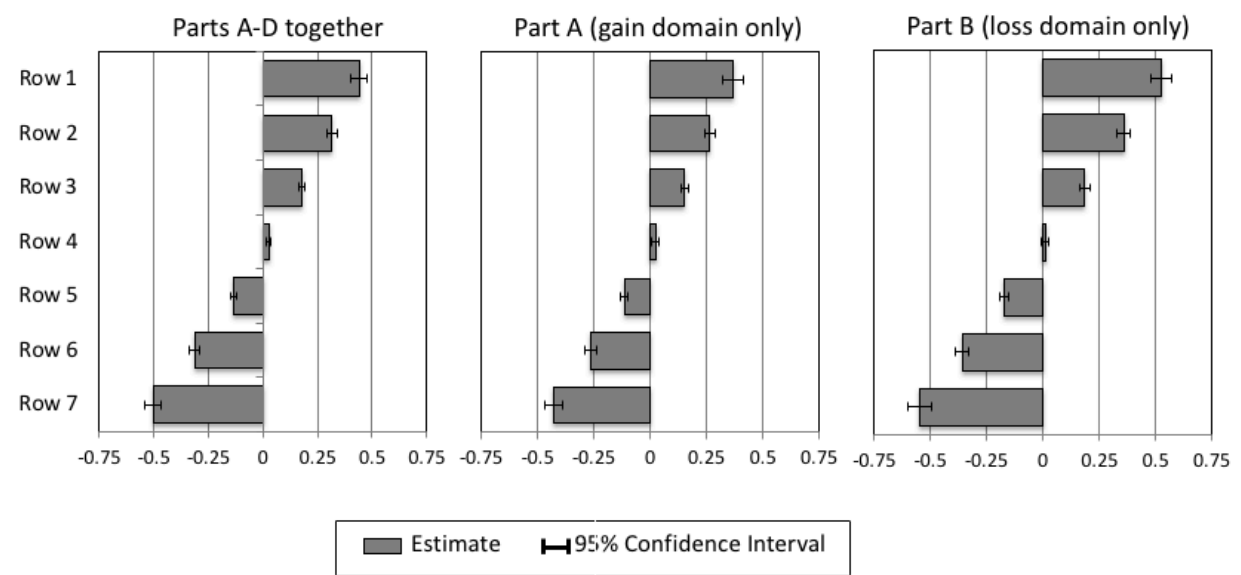

Online Appendix Figure 6.1. Implied estimates of the parameters for the compromise effect $c_{i}$ as a function of the row $i$ in which a choice appears. This figure is analogous to Figure 2 in the main text, except that the results were obtained by estimating the CPT model with CARA utility. 

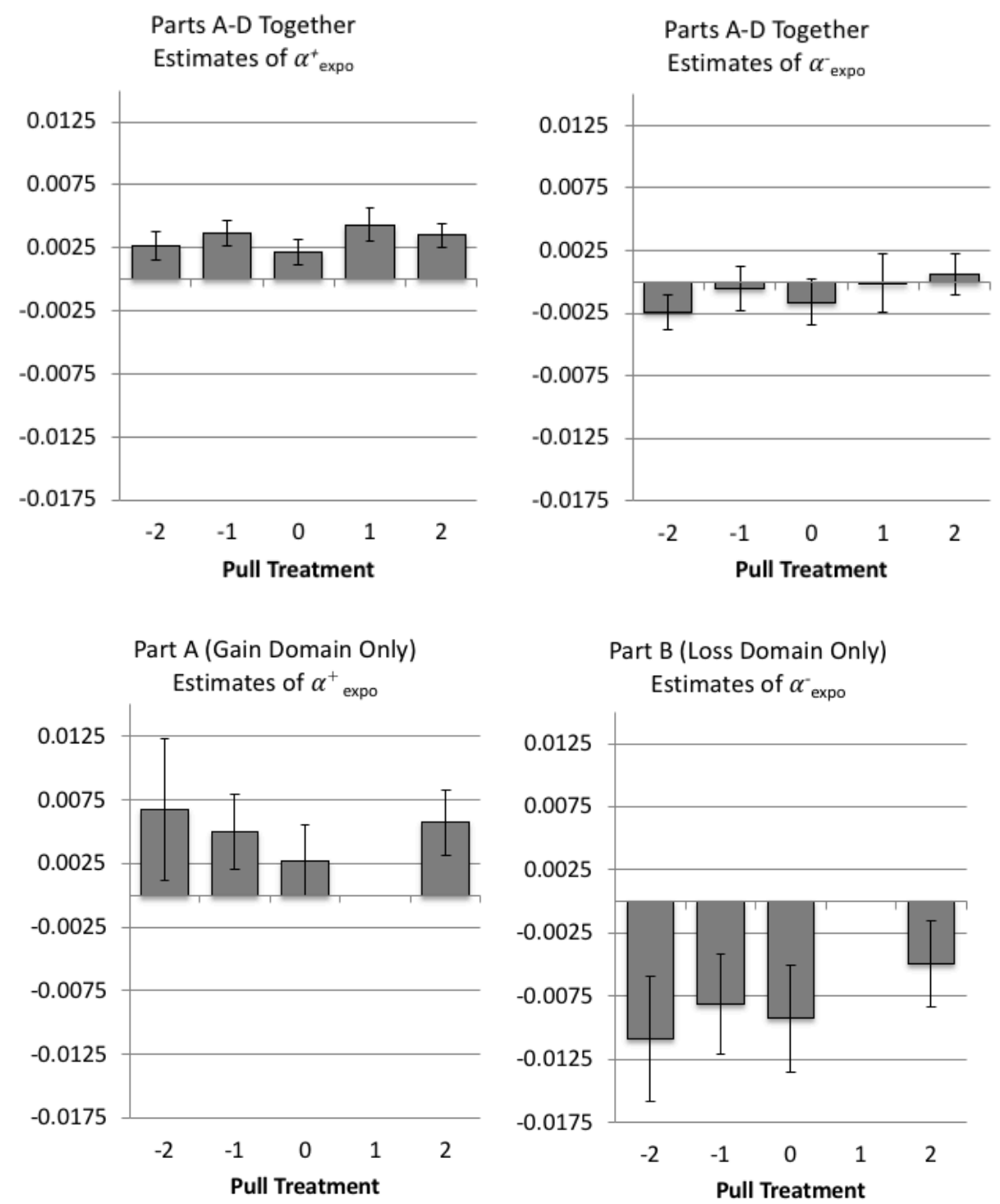

Estimate $\longmapsto 95 \%$ Confidence Interval

Online Appendix Figure 6.2. Estimates of $\alpha_{\text {expo }}^{+}$and $\alpha_{\text {expo }}^{-}$by Pull treatment, from the model with the compromise effect. This figure is analogous to Figure 3 in the main text, except that the results were obtained by estimating the CPT model with CARA utility. We omit the estimates for Pull Treatment 1 in the bottom two panels (Part A only and Part B only) because the MLE algorithm did not converge for these. 


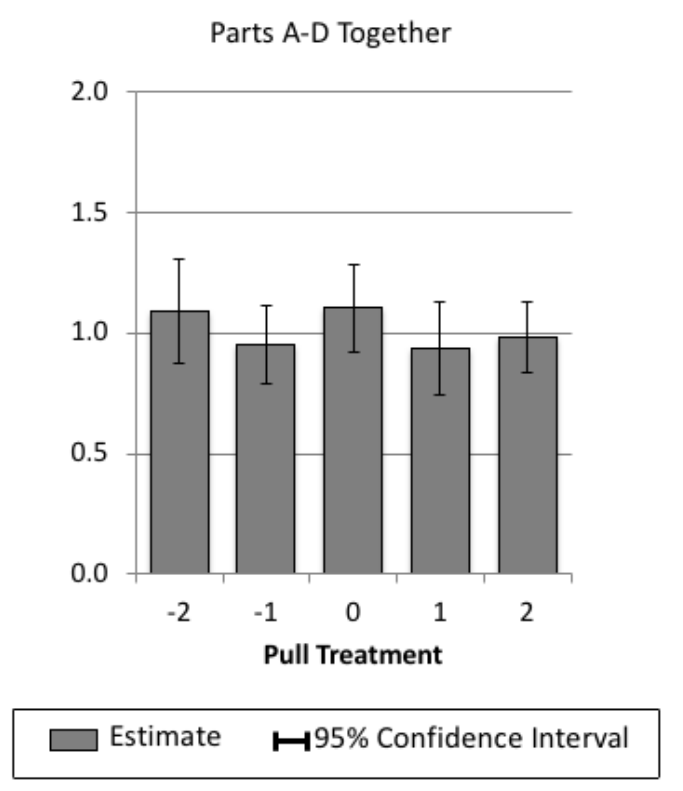

Online Appendix Figure 6.3. Estimates of $\lambda$ by Pull treatment from the model with the compromise effect, for Parts A-D together. This figure is analogous to Figure 4 in the main text, except that the results were obtained by estimating the CPT model with CARA utility. 

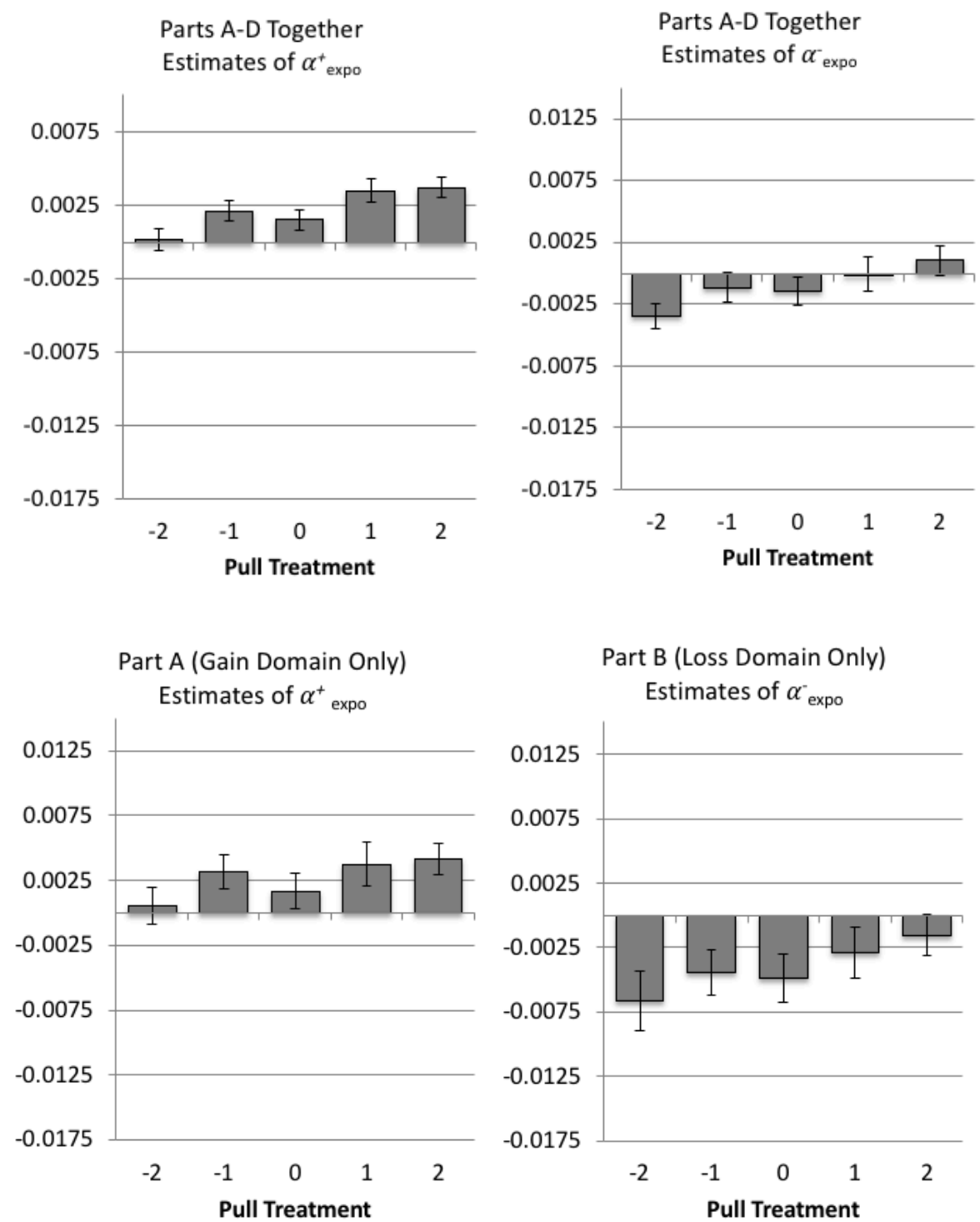

Estimate $\mapsto 95 \%$ Confidence Interval

Online Appendix Figure 6.4. Estimates of $\alpha_{\text {expo }}^{+}$and $\alpha_{\text {expo }}^{-}$by Pull treatment, from the model without the compromise effect. This figure is analogous to Figure 5 in the main text, except that the results were obtained by estimating the CPT model with CARA utility. 


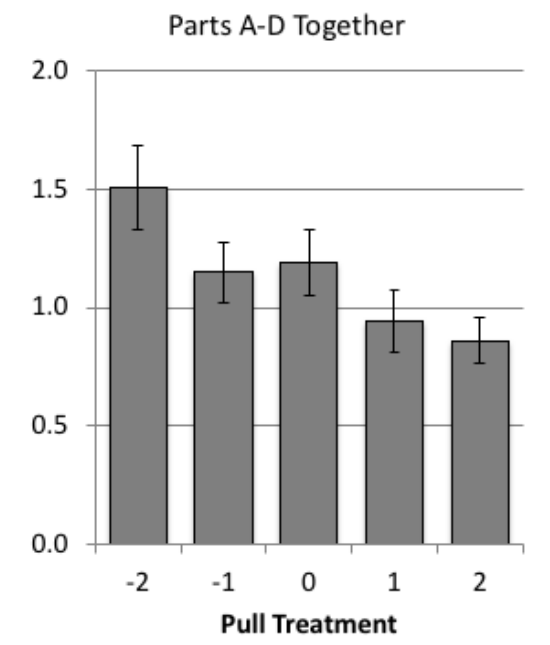

Estimate $\mapsto 95 \%$ Confidence Interval

Online Appendix Figure 6.5. Estimates of $\lambda$ by Pull treatment from the model without the compromise effect, for Parts A-D together. This figure is analogous to Figure 6 in the main text, except that the results were obtained by estimating the CPT model with CARA utility. 


\section{Online Appendix Table 6.1. ML Estimates of Selected Parameters in the Model with the Compromise Effect}

\begin{tabular}{clll}
\hline \hline & $\begin{array}{l}\text { Parts A-D } \\
\text { Together }\end{array}$ & $\begin{array}{l}\text { Part A (Gain } \\
\text { Domain Only) }\end{array}$ & $\begin{array}{l}\text { Part B (Loss } \\
\text { Domain Only) }\end{array}$ \\
\hline \hline$\alpha_{\text {expo }}^{+}$ & $0.0034^{* * *}$ & $0.0054^{* * *}$ & \\
& $(0.0002)$ & $(0.0007)$ & \\
$\alpha_{\text {expo }}^{-}$ & $-0.0008^{*}$ & & $-0.0065^{* * *}$ \\
& $(0.0004)$ & & $(0.0008)$ \\
$\lambda$ & $0.992^{* * *}$ & & \\
& $(0.040)$ & & \\
$\alpha, \alpha^{+}, \alpha^{-}$ & $0.638^{* * *}$ & $0.592^{* * *}$ & $0.694^{* * *}$ \\
& $(0.016)$ & $(0.016)$ & $(0.019)$ \\
$\beta, \beta^{+}, \beta^{-}$ & $1.331^{* * *}$ & $1.184^{* * *}$ & $1.72^{* * *}$ \\
& $(0.027)$ & $(0.036)$ & $(0.074)$ \\
$\pi_{1}$ & $-0.105^{* * *}$ & $-0.084^{* * *}$ & $-0.160^{* * *}$ \\
& $(.012)$ & $(0.018)$ & $(0.018)$ \\
$\pi_{2}$ & $-0.007^{* * *}$ & $-0.006^{* * *}$ & -0.002 \\
& $(0.001)$ & $(0.002)$ & $(0.002)$ \\
Log-likelihood & $-55,410$ & $-24,321$ & $-25,294$ \\
Wald test for $\pi_{1}, \pi_{2}$ & $p<1 \times 10^{-151}$ & $p<1 \times 10^{-95}$ & $p<1 \times 10^{-126}$ \\
Parameters & 20 & 10 & 10 \\
Individuals & 493 & 493 & 493 \\
Observations & 30,566 & 13,804 & 13,804 \\
\hline \hline
\end{tabular}

NOTE: Standard errors are clustered by participant. The log-likelihood statistic is for the model without clustering. The Wald test is for the joint significance of $\pi_{1}$ and $\pi_{2}$. This table is analogous to Table 1 in the main text, except that the results were obtained by estimating the CPT model with CARA utility.

* significant at $10 \%$ level; ** significant at $5 \%$ level; *** significant at $1 \%$ level. 


\section{Online Appendix Table 6.2. ML Estimates of Selected Parameters in the Parameterized Model with the Compromise Effect}

\begin{tabular}{|c|c|c|c|c|}
\hline & & $\begin{array}{l}\text { Parts A-D } \\
\text { Together }\end{array}$ & $\begin{array}{l}\text { Part A (Gain } \\
\text { Domain Only) }\end{array}$ & $\begin{array}{l}\text { Part B (Loss } \\
\text { Domain Only) }\end{array}$ \\
\hline \multirow[t]{3}{*}{$\alpha_{\text {expo }}^{+}$} & $\overline{\alpha_{\text {expo }, 0}^{+}}$ & $\begin{array}{l}0.0034 * * * \\
(0.0004)\end{array}$ & $\begin{array}{l}0.0067 * * * \\
(0.0012)\end{array}$ & \\
\hline & $\phi_{1}^{\alpha_{\text {expo }}^{+}}$ & $\begin{array}{l}0.0001 \\
(0.0002)\end{array}$ & $\begin{array}{l}-0.0010 \\
(0.0006)\end{array}$ & \\
\hline & $\phi_{2}^{\alpha_{\text {expo }}^{+}}$ & $\begin{array}{l}-0.0001 \\
(0.0006)\end{array}$ & $\begin{array}{l}-0.0014 \\
(0.0014)\end{array}$ & \\
\hline \multirow[t]{3}{*}{$\alpha_{\text {expo }}^{-}$} & $\alpha_{\text {expo }, 0}^{-}$ & $\begin{array}{l}-0.0003 \\
(0.0006)\end{array}$ & & $\begin{array}{l}-0.0059 * * * \\
(0.0010)\end{array}$ \\
\hline & $\phi_{1}^{\alpha \overline{e x p o}}$ & $\begin{array}{l}0.0006^{* *} \\
(0.0003)\end{array}$ & & $\begin{array}{l}0.0006 \\
(0.0004)\end{array}$ \\
\hline & $\phi_{2}^{\alpha_{\text {expo }}^{-}}$ & $\begin{array}{l}-0.0009 \\
(0.0008)\end{array}$ & & $\begin{array}{l}-0.0009 \\
(0.0010)\end{array}$ \\
\hline \multirow[t]{3}{*}{$\lambda$} & $\lambda_{0}$ & $\begin{array}{l}0.973 * * * \\
(0.056)\end{array}$ & & \\
\hline & $\phi_{1}^{\lambda}$ & $\begin{array}{l}-0.015 \\
(0.027)\end{array}$ & & \\
\hline & $\phi_{2}^{\lambda}$ & $\begin{array}{l}0.037 \\
(0.081)\end{array}$ & & \\
\hline$\alpha_{0}, \alpha_{0}^{+}, \alpha_{0}^{-}$ & & $\begin{array}{l}0.572 * * * \\
(0.020)\end{array}$ & $\begin{array}{l}0.533 * * * \\
(0.021)\end{array}$ & $\begin{array}{l}0.626 * * * \\
(0.025)\end{array}$ \\
\hline$\beta_{0}, \beta_{0}^{+}, \beta_{0}^{-}$ & & $\begin{array}{l}1.35^{* * *} \\
(0.040)\end{array}$ & $\begin{array}{l}1.150 * * * \\
(0.057)\end{array}$ & $\begin{array}{l}1.74 * * * \\
(0.103)\end{array}$ \\
\hline$\pi_{1}$ & & $\begin{array}{l}-0.102 * * * \\
(0.012)\end{array}$ & $\begin{array}{l}-0.094 * * * \\
(0.018)\end{array}$ & $\begin{array}{l}-0.155^{* * *} \\
(0.018)\end{array}$ \\
\hline$\pi_{2}$ & & $\begin{array}{l}-0.007 * * * \\
(0.001)\end{array}$ & $\begin{array}{l}-0.005^{* * *} \\
(0.002)\end{array}$ & $\begin{array}{l}-0.003 \\
(0.002)\end{array}$ \\
\hline Log-likelihood & & $-55,246$ & $-24,234$ & \\
\hline Wald test for $\pi_{1}, \pi_{2}$ & & $p<1 \times 10^{-146}$ & $p<1 \times 10^{-105}$ & $p<1 \times 10^{-121}$ \\
\hline Parameters & & 56 & 26 & 26 \\
\hline Individuals & & 493 & 493 & 493 \\
\hline Observations & & 30,566 & 13,804 & 13,804 \\
\hline
\end{tabular}

NOTE: Standard errors are clustered by participant. The log-likelihood statistic is for the model without clustering. The Wald test is for the joint significance of $\pi_{1}$ and $\pi_{2}$. This table is analogous to Table 2 in the main text, except that the results were obtained by estimating the CPT model with CARA utility.

$*$ significant at $10 \%$ level; ** significant at $5 \%$ level; *** significant at $1 \%$ level. 


\section{Online Appendix Table 6.3. ML Estimates of Selected Parameters in the Model Without the Compromise Effect}

\begin{tabular}{clll}
\hline \hline & $\begin{array}{l}\text { Parts A-D } \\
\text { Together }\end{array}$ & $\begin{array}{l}\text { Part A (Gain } \\
\text { Domain Only) }\end{array}$ & $\begin{array}{l}\text { Part B (Loss } \\
\text { Domain Only) }\end{array}$ \\
\hline \hline$\alpha_{\text {expo }}^{+}$ & $\begin{array}{l}0.0021^{* * *} \\
(0.0002)\end{array}$ & $\begin{array}{l}0.0023^{* * *} \\
(0.0003)\end{array}$ & \\
$\alpha_{\text {expo }}^{-}$ & $-0.0013^{* * *}$ & & $-0.0045^{* * *}$ \\
& $(0.0003)$ & & $(0.0004)$ \\
$\lambda$ & $1.098^{* * *}$ & & \\
$\alpha, \alpha^{+}, \alpha^{-}$ & $(0.032)$ & & $0.632^{* * *}$ \\
$\beta, \beta^{+}, \beta^{-}$ & $0.587 * * *$ & $.554 * * *$ & $(0.012)$ \\
& $(0.010)$ & $(0.011)$ & $1.503 * * *$ \\
Log-likelihood & $1.309 * * *$ & $1.260 * * *$ & $(0.036)$ \\
Parameters & $(0.017)$ & $(0.023)$ & $-27,953$ \\
Individuals & $-60,099$ & $-26,197$ & 8 \\
Observations & 18 & 8 & 493 \\
\hline \hline
\end{tabular}

NOTE: Standard errors are clustered by participant. The log-likelihood statistic is for the model without clustering. This table is analogous to Table 3 in the main text, except that the results were obtained by estimating the CPT model with CARA utility.

$*$ significant at $10 \%$ level; $* *$ significant at $5 \%$ level; $* * *$ significant at $1 \%$ level. 
Online Appendix Table 6.4. ML Estimates of Selected Parameters in the Parameterized Model Without the Compromise Effect

\begin{tabular}{|c|c|c|c|c|}
\hline & & $\begin{array}{l}\text { Parts A-D } \\
\text { Together } \\
\end{array}$ & $\begin{array}{l}\text { Part A (Gain } \\
\text { Domain Only) } \\
\end{array}$ & $\begin{array}{l}\text { Part B (Loss } \\
\text { Domain Only) } \\
\end{array}$ \\
\hline \multirow[t]{3}{*}{$\overline{\alpha_{\text {expo }}^{+}}$} & $\alpha_{\text {expo }, 0}^{+}$ & $\begin{array}{l}0.0023 * * * \\
(0.0003)\end{array}$ & $\begin{array}{l}0.0031 * * * \\
(0.0004)\end{array}$ & \\
\hline & $\phi_{1}^{\alpha_{e x p o}^{+}}$ & $\begin{array}{l}0.0008^{* * *} \\
(0.0001)\end{array}$ & $\begin{array}{l}0.0010 * * * \\
(0.0003)\end{array}$ & \\
\hline & $\phi_{2}^{\alpha_{e x p o}^{+}}$ & $\begin{array}{l}-0.0001 \\
(0.0004)\end{array}$ & $\begin{array}{l}-0.0012 * \\
(0.0006)\end{array}$ & \\
\hline \multirow[t]{3}{*}{$\alpha_{\text {expo }}^{-}$} & $\alpha_{\text {expo }, 0}^{-}$ & $\begin{array}{l}-0.0006^{*} \\
(0.0004)\end{array}$ & & $\begin{array}{l}-0.0036 * * * \\
(0.0005)\end{array}$ \\
\hline & $\phi_{1}^{\alpha_{\text {expo }}^{-}}$ & $\begin{array}{l}0.0009^{* * *} \\
(0.0002)\end{array}$ & & $\begin{array}{l}0.0011^{* * *} \\
(0.0002)\end{array}$ \\
\hline & $\phi_{2}^{\alpha_{\text {expo }}^{-}}$ & $\begin{array}{l}-0.0008^{*} \\
(0.0005)\end{array}$ & & $\begin{array}{l}-0.0009 \\
(0.0006)\end{array}$ \\
\hline \multirow[t]{3}{*}{$\lambda$} & $\lambda_{0}$ & $\begin{array}{l}1.115^{* * *} \\
(0.042)\end{array}$ & & \\
\hline & $\phi_{1}^{\lambda}$ & $\begin{array}{l}-0.141 * * * \\
(0.021)\end{array}$ & & \\
\hline & $\phi_{2}^{\lambda}$ & $\begin{array}{l}0.021 \\
(0.059)\end{array}$ & & \\
\hline$\alpha_{0}, \alpha_{0}^{+}, \alpha_{0}^{-}$ & & $\begin{array}{l}0.547 * * * \\
(0.013)\end{array}$ & $\begin{array}{l}0.514 * * * \\
(0.015)\end{array}$ & $\begin{array}{l}0.590 * * * \\
(0.016)\end{array}$ \\
\hline$\beta_{0}, \beta_{0}^{+}, \beta_{0}^{-}$ & & $\begin{array}{l}1.312 * * * \\
(0.024)\end{array}$ & $\begin{array}{l}1.245^{* * *} \\
(0.033)\end{array}$ & $\begin{array}{l}1.49 * * * \\
(0.047)\end{array}$ \\
\hline Log-likelihood & & $-59,571$ & $-26,003$ & $-27,712$ \\
\hline Parameters & & 54 & 24 & 24 \\
\hline Individuals & & 493 & 493 & 493 \\
\hline Observations & & 30,566 & 13,804 & 13,804 \\
\hline
\end{tabular}

NOTE: Standard errors are clustered by participant. The log-likelihood statistic is for the model without clustering. This table is analogous to Table 4 in the main text, except that the results were obtained by estimating the CPT model with CARA utility.

* significant at $10 \%$ level; ** significant at $5 \%$ level; *** significant at $1 \%$ level. 


\section{Results of Robustness Check with CPT Model with Expo-Power Utility}

As a robustness check, we estimated the CPT model with expo-power utility (Saha 1993), $u(x)=\frac{1-e^{-\alpha_{e-p} x^{1-\gamma_{e-p}}}}{\alpha_{e-p}}$, instead of with CRRA utility. As in the baseline model, we used the Prelec (1998) probability weighting function.

Online Appendix Figures 9.1-9.9 and Online Appendix Tables 9.1-9.4 below are analogous to Figures 2-6 and Tables 1-4 in the main text, respectively, except that the results were obtained by estimating the CPT model with expo-power utility.

It is difficult to interpret the effects of the Pull treatment on the parameters of expo-power utility because both $\gamma_{e-p}$ and $\alpha_{e-p}$ capture risk aversion. To see this, note that the ArrowPratt coefficient of relative risk aversion is $\frac{-u^{\prime \prime}(x) x}{u^{\prime}(x)}=\gamma_{e-p}+\alpha_{e-p}\left(1-\gamma_{e-p}\right) x^{1-\gamma_{e-p}}$, which depends on both $\gamma_{e-p}$ and $\alpha_{e-p}$. As a result, $\gamma_{e-p}$ and $\alpha_{e-p}$ may move together across Pull treatments in complicated ways, and there is no clear theoretical relationship between $\gamma_{e-p}, \alpha_{e-p}$ and Pull treatment. For that reason, in Online Appendix Figures 9.29.4 and 9.6-9.8, we report estimates of the coefficient of relative risk aversion with $x=$ 10,50 , and 200 by Pull treatment (instead of estimates of $\gamma_{e-p}$ and $\alpha_{e-p}$ by Pull treatment). Also, in Online Appendix Tables 7.2 and 7.4, we only report the results of parameterized model for Parts A-D together, since it is only meaningful to interpret the effect of the Pull treatment on the parameter $\lambda$ (and we can only estimate $\lambda$ using data from Parts A-D together). 

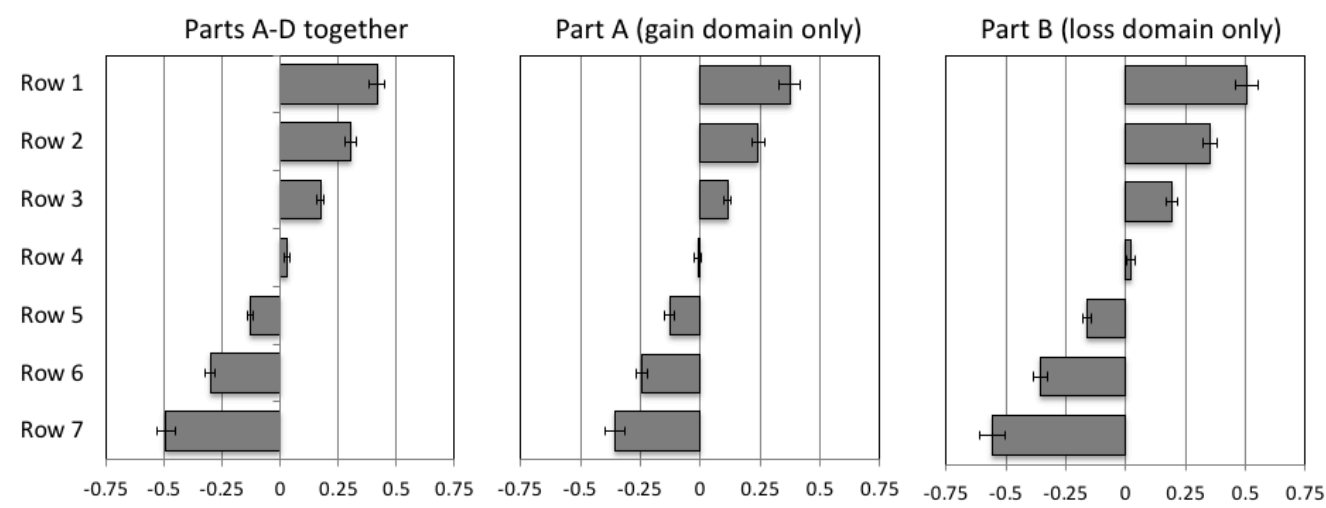

Estimate $\mapsto 95 \%$ Confidence Interval

Online Appendix Figure 7.1. Implied estimates of the parameters for the compromise effect $c_{i}$ as a function of the row $i$ in which a choice appears. This figure is analogous to Figure 2 in the main text, except that the results were obtained by estimating the CPT model with expo-power utility. 


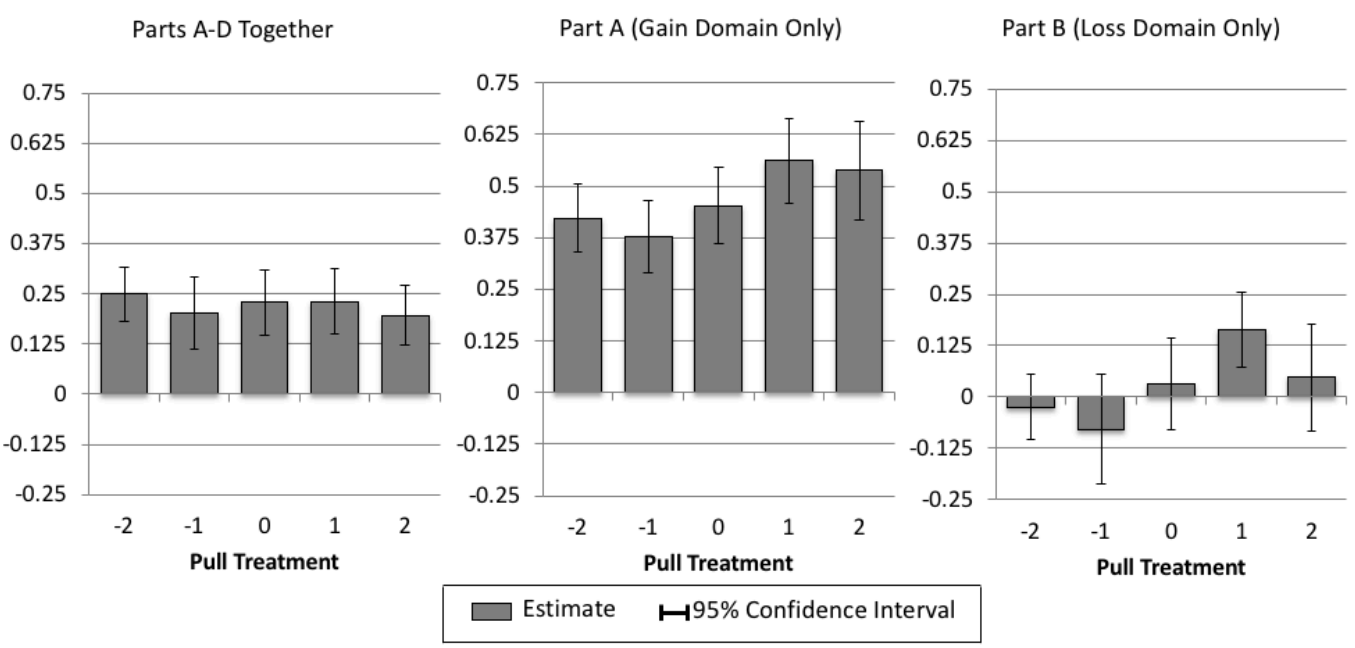

Online Appendix Figure 7.2. Estimates of the Arrow-Pratt coefficient of relative risk aversion (with $\mathrm{x}=10$ ) by Pull treatment, from the model with the compromise effect. This figure is analogous to Figure 3 in the main text, except that the results were obtained by estimating the CPT model with expo-power utility. We report the Arrow-Pratt coefficient of relative risk aversion instead of the estimates of $\gamma_{e-p}$ and $\alpha_{e-p}$ because both $\gamma_{e-p}$ and $\alpha_{e-p}$ capture risk aversion and may move together in complicated ways across Pull treatments.
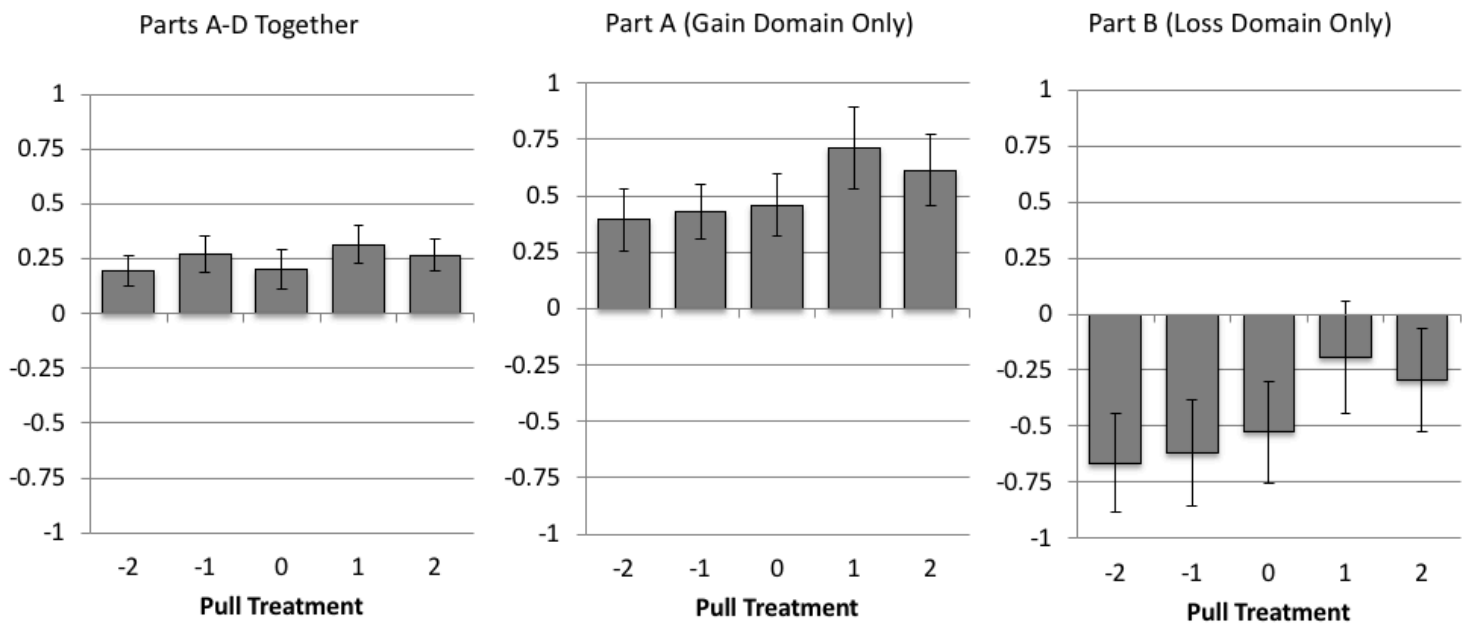

Estimate $\mapsto 95 \%$ Confidence Interval

Online Appendix Figure 7.3. Estimates of the Arrow-Pratt coefficient of relative risk aversion (with $\mathrm{x}=50$ ) by Pull treatment, from the model with the compromise effect. This figure is analogous to Figure 3 in the main text, except that the results were obtained by estimating the CPT model with expo-power utility. We report the Arrow-Pratt coefficient of relative risk aversion instead of the estimates of $\gamma_{e-p}$ and $\alpha_{e-p}$ because both $\gamma_{e-p}$ and $\alpha_{e-p}$ capture risk aversion and may move together in complicated ways across Pull treatments. 

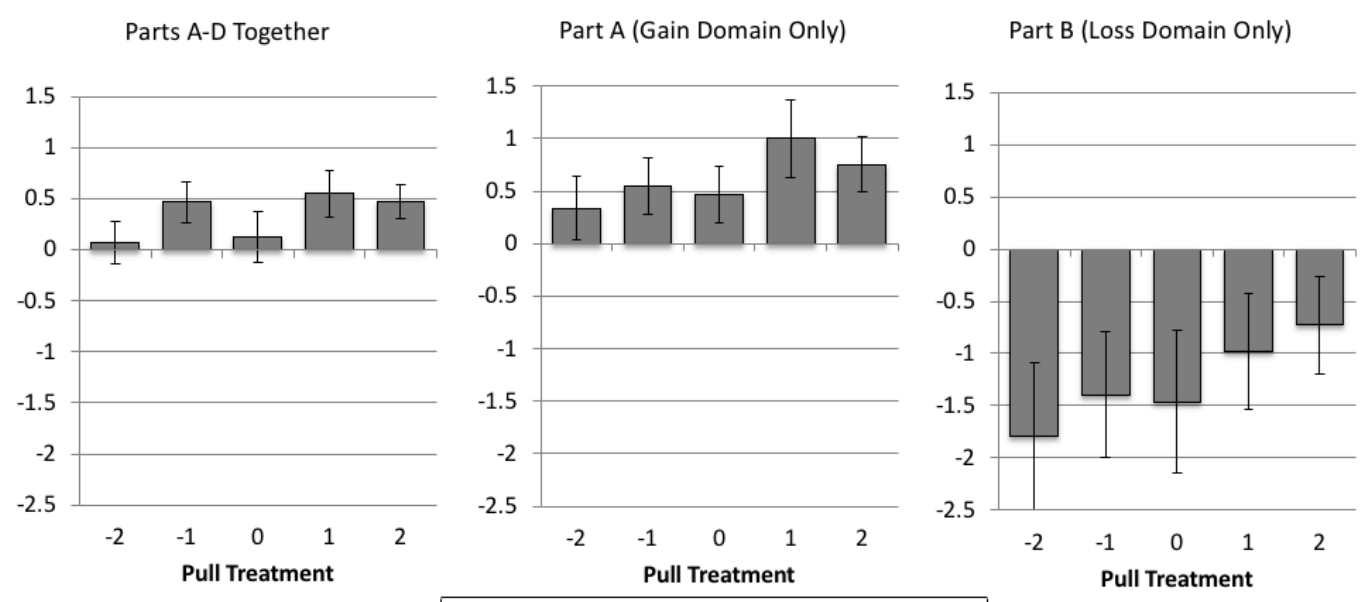

$\square$ Estimate $\quad \mapsto 95 \%$ Confidence Interval

Online Appendix Figure 7.4. Estimates of the Arrow-Pratt coefficient of relative risk aversion (with $\mathrm{x}=200$ ) by Pull treatment, from the model with the compromise effect. This figure is analogous to Figure 3 in the main text, except that the results were obtained by estimating the CPT model with expo-power utility. We report the Arrow-Pratt coefficient of relative risk aversion instead of the estimates of $\gamma_{e-p}$ and $\alpha_{e-p}$ because both $\gamma_{e-p}$ and $\alpha_{e-p}$ capture risk aversion and may move together in complicated ways across Pull treatments. 
Parts A-D Together

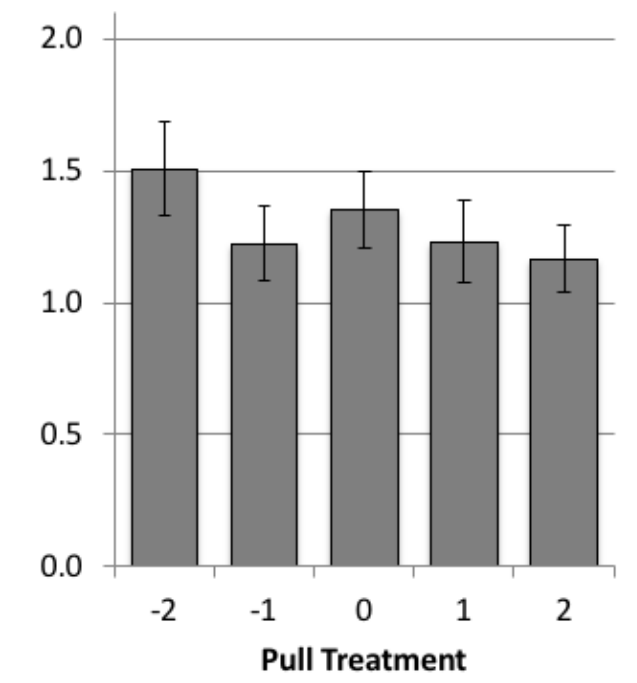

$\square$ Estimate $\mapsto 95 \%$ Confidence Interval

Online Appendix Figure 7.5. Estimates of $\lambda$ by Pull treatment from the model with the compromise effect, for Parts A-D together. This figure is analogous to Figure 4 in the main text, except that the results were obtained by estimating the CPT model with CARA utility. 


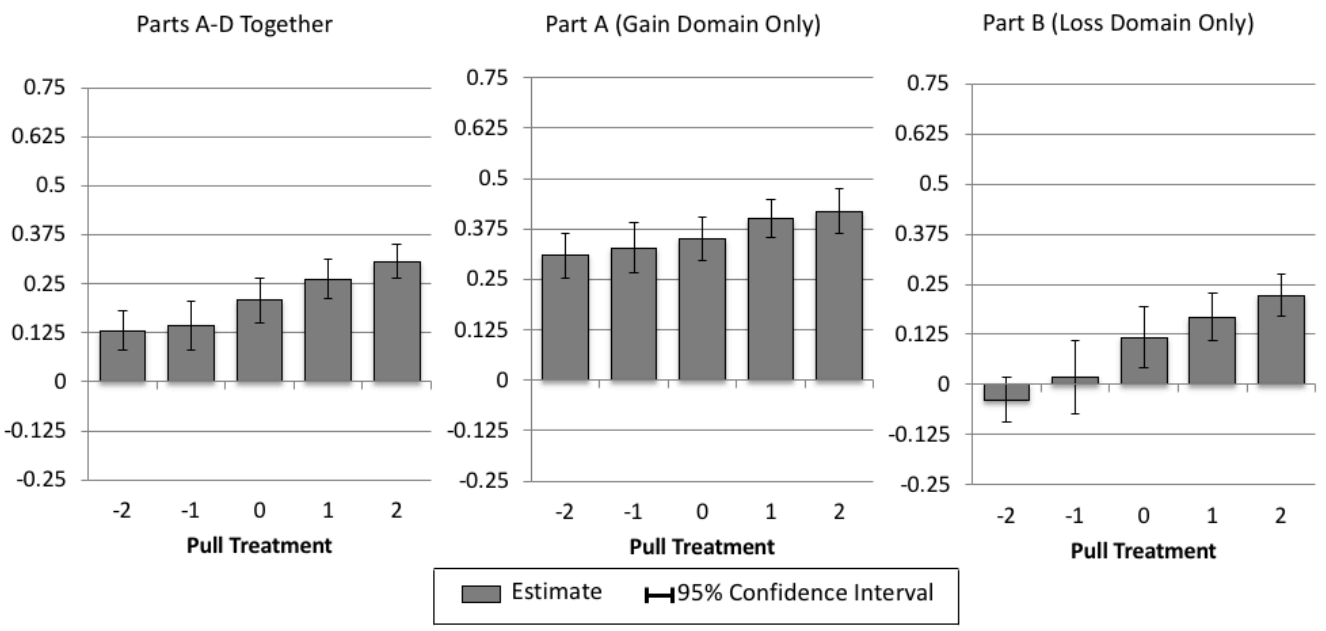

Online Appendix Figure 7.6. Estimates of the Arrow-Pratt coefficient of relative risk aversion (with $\mathrm{x}=10$ ) by Pull treatment, from the model without the compromise effect. This figure is analogous to Figure 5 in the main text, except that the results were obtained by estimating the CPT model with expo-power utility. We report the Arrow-Pratt coefficient of relative risk aversion instead of the estimates of $\gamma_{e-p}$ and $\alpha_{e-p}$ because both $\gamma_{e-p}$ and $\alpha_{e-p}$ capture risk aversion and may move together in complicated ways across Pull treatments.
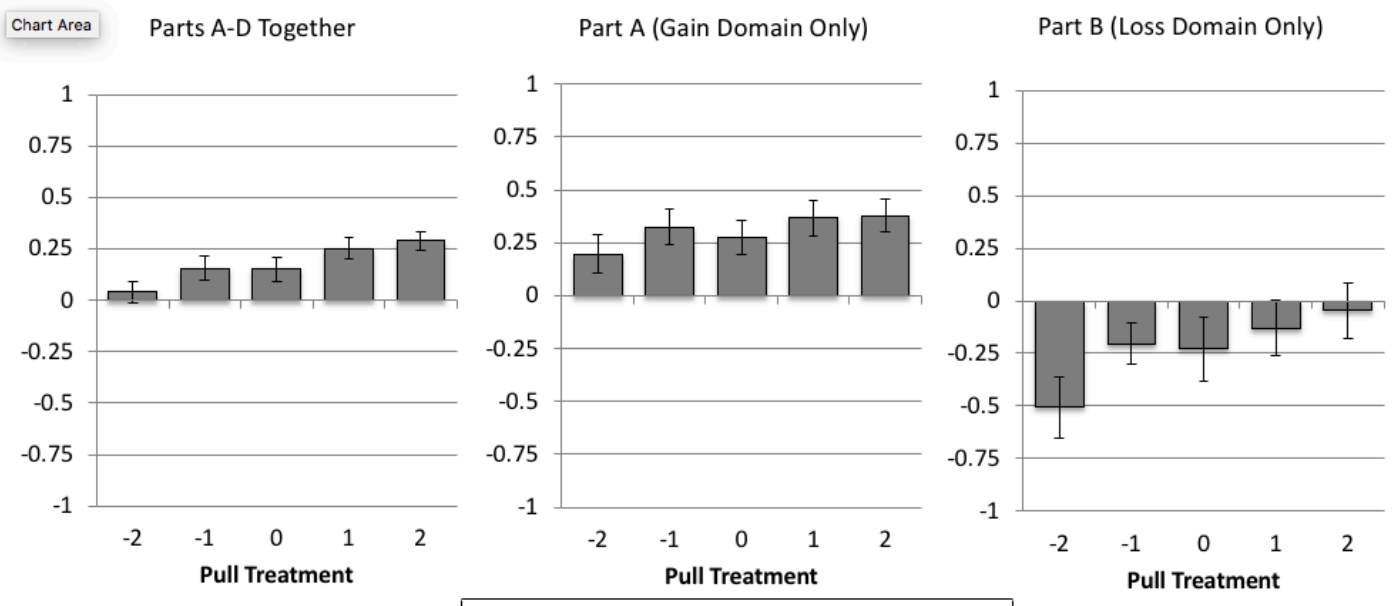

$\square$ Estimate $\mapsto 95 \%$ Confidence Interval

Online Appendix Figure 7.7. Estimates of the Arrow-Pratt coefficient of relative risk aversion (with $\mathrm{x}=50$ ) by Pull treatment, from the model without the compromise effect. This figure is analogous to Figure 5 in the main text, except that the results were obtained by estimating the CPT model with expo-power utility. We report the Arrow-Pratt coefficient of relative risk aversion instead of the estimates of $\gamma_{e-p}$ and $\alpha_{e-p}$ because both $\gamma_{e-p}$ and $\alpha_{e-p}$ capture risk aversion and may move together in complicated ways across Pull treatments. 

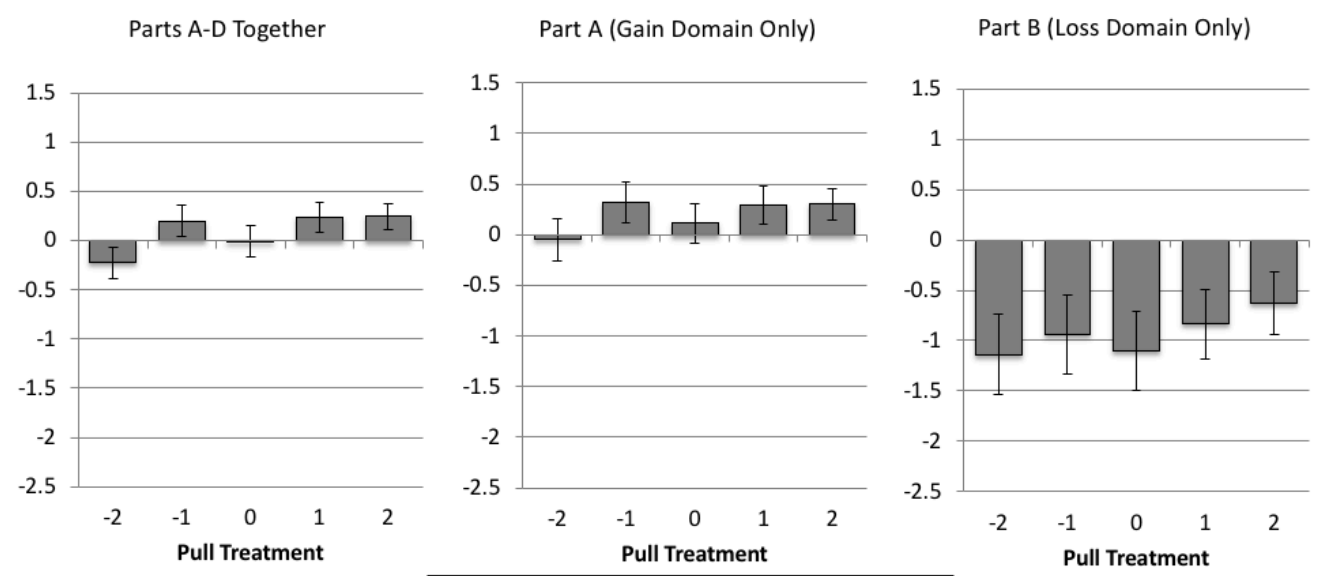

$\square$ Estimate $\quad \mapsto^{95 \%}$ Confidence Interval

Online Appendix Figure 7.8. Estimates of the Arrow-Pratt coefficient of relative risk aversion (with $\mathrm{x}=200$ ) by Pull treatment, from the model without the compromise effect. This figure is analogous to Figure 5 in the main text, except that the results were obtained by estimating the CPT model with expo-power utility. We report the Arrow-Pratt coefficient of relative risk aversion instead of the estimates of $\gamma_{e-p}$ and $\alpha_{e-p}$ because both $\gamma_{e-p}$ and $\alpha_{e-p}$ capture risk aversion and may move together in complicated ways across Pull treatments. 


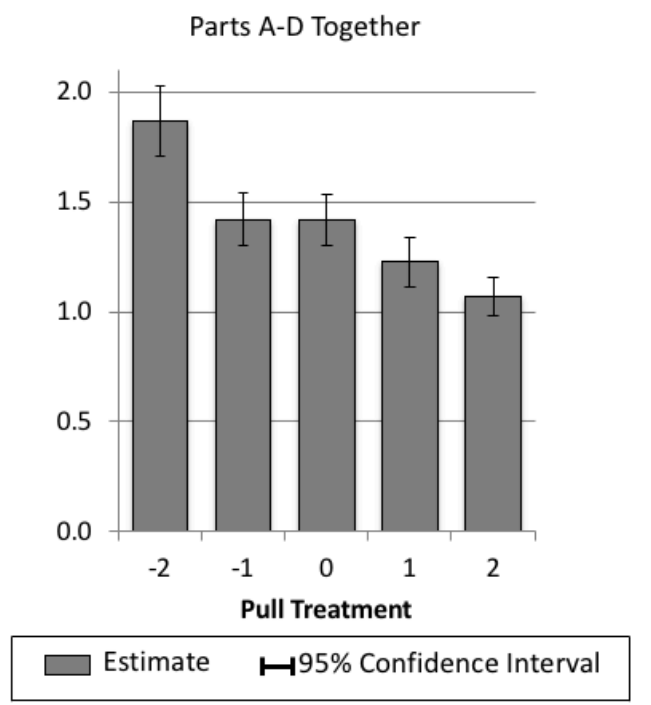

Online Appendix Figure 7.9. Estimates of $\lambda$ by Pull treatment from the model without the compromise effect, for Parts A-D together. This figure is analogous to Figure 6 in the main text, except that the results were obtained by estimating the CPT model with expopower utility. 


\section{Online Appendix Table 7.1. ML Estimates of Selected Parameters in the Model with the Compromise Effect}

\begin{tabular}{clll}
\hline \hline & $\begin{array}{l}\text { Parts A-D } \\
\text { Together }\end{array}$ & $\begin{array}{l}\text { Part A (Gain } \\
\text { Domain Only) }\end{array}$ & $\begin{array}{l}\text { Part B (Loss } \\
\text { Domain Only) }\end{array}$ \\
\hline \hline$\gamma_{e-p}, \gamma_{e-p}^{+}, \gamma_{e-p}^{-}$ & $0.219^{* * *}$ & $0.427^{* * *}$ & $0.677^{* * *}$ \\
& $(0.020)$ & $(0.020)$ & $(0.044)$ \\
$\alpha_{e-p}, \alpha_{e-p}^{+}, \alpha_{e-p}^{-}$ & $0.0025^{* *}$ & $0.0116^{* *}$ & $-0.9687^{* * *}$ \\
& $(0.0010)$ & $(0.0054)$ & $(0.3114)$ \\
$\lambda$ & $1.288^{* * *}$ & & \\
& $(0.034)$ & & \\
$\alpha, \alpha^{+}, \alpha^{-}$ & $0.622^{* * *}$ & $0.566^{* * *}$ & $0.679^{* * *}$ \\
& $(0.015)$ & $(0.015)$ & $(0.018)$ \\
$\beta, \beta^{+}, \beta^{-}$ & $1.112^{* * *}$ & $0.837^{* * *}$ & $1.67^{* * *}$ \\
& $(0.025)$ & $(0.037)$ & $(0.075)$ \\
$\pi_{1}$ & $-0.091^{* * *}$ & $-0.137^{* * *}$ & $-0.136^{* * *}$ \\
& $(0.012)$ & $(0.018)$ & $(0.019)$ \\
$\pi_{2}$ & $-0.008^{* * *}$ & 0.002 & $-0.005^{* *}$ \\
& $(0.001)$ & $(0.002)$ & $(0.002)$ \\
Log-likelihood & $-55,374$ & $-23,912$ & $-25,264$ \\
Wald test for $\pi_{1}, \pi_{2}$ & $p<1 \times 10^{-146}$ & $p<1 \times 10^{-79}$ & $p<1 \times 10^{-123}$ \\
Parameters & 20 & 11 & 11 \\
Individuals & 493 & 493 & 493 \\
Observations & 30,566 & 13,804 & 13,804 \\
\hline \hline
\end{tabular}

NOTE: Standard errors are clustered by participant. The log-likelihood statistic is for the model without clustering. The Wald test is for the joint significance of $\pi_{1}$ and $\pi_{2}$. This table is analogous to Table 1 in the main text, except that the results were obtained by estimating the CPT model with expopower utility.

* significant at $10 \%$ level; ** significant at $5 \%$ level; *** significant at $1 \%$ level. 


\section{Online Appendix Table 7.2. ML Estimates of Selected Parameters in the Parameterized Model with the Compromise Effect}

\begin{tabular}{cll}
\hline \hline & & $\begin{array}{l}\text { Parts A-D } \\
\text { Together }\end{array}$ \\
\hline \hline$\gamma_{e-p, 0}$ & & $0.175^{* * *}$ \\
& $(0.029)$ \\
$\alpha_{e-p, 0}$ & & $0.0032^{* * *}$ \\
& & $(0.0010)$ \\
$\lambda$ & $\lambda_{0}$ & $1.234^{* * *}$ \\
& & $(0.049)$ \\
& $\phi_{1}^{\lambda}$ & $-0.066^{* *}$ \\
& & $(0.026)$ \\
& $\phi_{2}^{\lambda}$ & 0.105 \\
$\alpha_{0}$ & & $(0.069)$ \\
& & $0.559^{* * *}$ \\
$\beta_{0}$ & & $(0.019)$ \\
& & $1.168^{* * *}$ \\
$\pi_{1}$ & & $(0.037)$ \\
& & $-0.089^{* * *}$ \\
$\pi_{2}$ & $(0.012)$ \\
& & $-0.008^{* * *}$ \\
Log-likelihood & & $(0.001)$ \\
Wald test for $\pi_{1}, \pi_{2}$ & & $-55,210$ \\
Parameters & $p<1 \times 10^{-138}$ \\
Individuals & 56 \\
Observations & 493 \\
\hline \hline
\end{tabular}

NOTE: Standard errors are clustered by participant. The log-likelihood statistic is for the model without clustering. The Wald test is for the joint significance of $\pi_{1}$ and $\pi_{2}$. This table is analogous to Table 2 in the main text, except that the results were obtained by estimating the CPT model with expopower utility. Both $\gamma_{e-p}$ and $\alpha_{e-p}$ capture risk aversion and may move together across Pull treatments in complicated ways, and as a result only estimates related to $\lambda$ are meaningful in the parameterized model with expo-power utility. We thus only report the results for Parts A-D together.

* significant at $10 \%$ level; ** significant at $5 \%$ level; *** significant at $1 \%$ level. 


\section{Online Appendix Table 7.3. ML Estimates of Selected Parameters in the Model Without the Compromise Effect}

\begin{tabular}{clll}
\hline \hline & $\begin{array}{l}\text { Parts A-D } \\
\text { Together }\end{array}$ & $\begin{array}{l}\text { Part A (Gain } \\
\text { Domain Only) }\end{array}$ & $\begin{array}{l}\text { Part B (Loss } \\
\text { Domain Only) }\end{array}$ \\
\hline \hline$\gamma_{e-p}, \gamma_{e-p}^{+}, \gamma_{e-p}^{-}$ & $0.244^{* * *}$ & $0.415^{* * *}$ & $0.331^{* * *}$ \\
$\alpha_{e-p}, \alpha_{e-p}^{+}, \alpha_{e-p}^{-}$ & $(0.016)$ & $(0.017)$ & $(0.103)$ \\
$\lambda$ & $-0.0046^{* * *}$ & $-0.0210^{* * *}$ & -0.0616 \\
$\lambda$ & $(0.0015)$ & $(0.0059)$ & $(0.0512)$ \\
$\alpha, \alpha^{+}, \alpha^{-}$ & $1.375^{* * *}$ & & \\
$\beta, \beta^{+}, \beta^{-}$ & $(0.030)$ & & $0.637 * * *$ \\
& $(0.574 * * *$ & $0.538^{* * *}$ & $(0.012)$ \\
Log-likelihood & $1.133^{* * *}$ & $(0.011)$ & $1.476^{* * *}$ \\
Parameters & $(0.016)$ & $0.992^{* * *}$ & $(0.051)$ \\
Individuals & $-59,933$ & $(0.022)$ & $-27,840$ \\
Observations & 18 & $-25,580$ & 9 \\
\hline \hline
\end{tabular}

NOTE: Standard errors are clustered by participant. The log-likelihood statistic is for the model without clustering. This table is analogous to Table 3 in the main text, except that the results were obtained by estimating the CPT model with expo-power utility.

$*$ significant at $10 \%$ level; $* *$ significant at $5 \%$ level; $* * *$ significant at $1 \%$ level. 


\section{Online Appendix Table 7.4. ML Estimates of Selected Parameters in the Parameterized Model Without the Compromise Effect}

\begin{tabular}{cll}
\hline \hline & $\begin{array}{l}\text { Parts A-D } \\
\text { Together }\end{array}$ \\
\hline \hline$\gamma_{e-p, 0}$ & & $0.213^{* * *}$ \\
& $(0.033)$ \\
$\alpha_{e-p, 0}$ & & -0.0014 \\
& & $(0.0032)$ \\
$\lambda$ & $\lambda_{0}$ & $1.33^{* * *}$ \\
& & $(0.045)$ \\
& $\phi_{1}^{\lambda}$ & $-0.154^{* * *}$ \\
& & $(0.021)$ \\
& $\phi_{2}^{\lambda}$ & $0.112^{*}$ \\
$\alpha_{0}$ & & $(0.061)$ \\
& & $0.535^{* * *}$ \\
$\beta_{0}$ & & $(0.012)$ \\
& & $1.149^{* * *}$ \\
& & $(0.022)$ \\
Log-likelihood & & $-59,414$ \\
Parameters & & 54 \\
Individuals & & 493 \\
Observations & & 30,566 \\
\hline \hline
\end{tabular}

NOTE: Standard errors are clustered by participant. The log-likelihood statistic is for the model without clustering. This table is analogous to Table 4 in the main text, except that the results were obtained by estimating the CPT model with expo-power utility. Both $\gamma_{e-p}$ and $\alpha_{e-p}$ capture risk aversion and may move together across Pull treatments in complicated ways, and as a result only estimates related to $\lambda$ are meaningful in the parameterized model with expo-power utility. We thus only report the results for Parts A-D together.

* significant at $10 \%$ level; ** significant at $5 \%$ level; *** significant at $1 \%$ level. 


\section{Numerical Estimates of the Parameters for the Compromise Effect $c_{i}$ as a Function of the Row $i$ in Which a Choice Appears}

Online Appendix Table 8.1 shows the numerical estimates of the parameters for the compromise effect $c_{i}$. These results are also shown graphically in Figure 2 of the paper.

Online Appendix Table 8.1. Estimates of the Parameters for the Compromise Effect $c_{i}$ in the Model with the Compromise Effect, as a Function of the Row $i$ in Which a Choice Appears

\begin{tabular}{llll}
\multicolumn{4}{c}{ Choice Appears } \\
\hline \hline & $\begin{array}{l}\text { Parts A-D } \\
\text { Together }\end{array}$ & $\begin{array}{l}\text { Part A (Gain } \\
\text { Domain Only) }\end{array}$ & $\begin{array}{l}\text { Part B (Loss } \\
\text { Domain only) }\end{array}$ \\
\hline \hline$c_{1}$ & $0.416^{* * *}$ & $0.371^{* * *}$ & $0.515^{* * *}$ \\
& $(0.018)$ & $(0.023)$ & $(0.023)$ \\
$c_{2}$ & $0.302^{* * *}$ & $0.242^{* * *}$ & $0.358^{* * *}$ \\
& $(0.012)$ & $(0.013)$ & $(0.015)$ \\
$c_{3}$ & $0.174^{* * *}$ & $0.116^{* * *}$ & 0.192 \\
& $(0.007)$ & $(0.008)$ & $(0.011)$ \\
$c_{4}$ & $0.030^{* * *}$ & -0.007 & $0.017^{*}$ \\
& $(0.005)$ & $(0.008)$ & $(0.009)$ \\
$c_{5}$ & $-0.128^{* * *}$ & $-0.126^{* * *}$ & $-0.166^{* * *}$ \\
& $(0.007)$ & $(0.009)$ & $(0.009)$ \\
$c_{6}$ & $-0.302^{* * *}$ & $-0.242^{* *}$ & $-0.358^{* * *}$ \\
& $(0.012)$ & $(0.013)$ & $(0.015)$ \\
$c_{7}$ & $-0.491^{* * *}$ & $-0.355^{* * *}$ & $-0.558^{* * *}$ \\
& $(0.020)$ & $(0.020)$ & $(0.027)$ \\
\hline \hline
\end{tabular}

NOTE: The estimates of $c_{i}$ were obtained by transforming the estimates of $\pi_{1}$ and $\pi_{2}$ from Table 1 of the paper, as described in the main text.

$*$ significant at $10 \%$ level; ** significant at $5 \%$ level; *** significant at $1 \%$ level. 


\section{Additional Information on the Analysis of the Demographic Correlates of the CPT Model and the Compromise Effect Parameters}

As mentioned in Section 8 of the main text, we analyzed the demographic correlates of the four key parameters of the CPT model $(\gamma, \lambda, \alpha, \beta)$ and of the two model parameters that capture the compromise effect $\left(\pi_{1}, \pi_{2}\right)$. Also as mentioned in Section 8 of the main text, in our baseline demographic specification, we estimate our CPT model with the compromise effect using data from Parts A-D together, with these six key model parameters specified as linear functions of a constant, age, sex, a dummy variable indicating whether one has a college degree, SAT Math score, the log of one's parents' combined annual income, as well as dummy variables to control for race. In other words, we substitute $\gamma$ in the utility function in equation (1) of the main text by:

$$
\begin{aligned}
\gamma= & \gamma_{0}+\phi_{\mathrm{age}}^{\gamma} \text { age }+\phi_{\mathrm{sex}}^{\gamma} \text { sex }+\phi_{\text {college }}^{\gamma} \text { college }+\phi_{\mathrm{SAT}}^{\gamma} S A T \_M a t h \\
& +\phi_{\text {inc. }}^{\gamma} \log (\text { parental_income })+\phi_{\text {other }}^{\gamma} \text { Other_variables },
\end{aligned}
$$

where Other_variables includes the dummy variables that control for race as well as dummy variables that indicate missing observations for each variable that has missing observations. We also substituted $\lambda, \alpha, \beta, \pi_{1}$, and $\pi_{2}$ with analogous parametrized equations.

In addition, we estimated several specifications to verify the robustness of the results from our baseline demographic specification. First, we estimated the baseline demographic specification again, but using data from Part A only, and then using data from Part B only. Second, we estimated a specification akin to the baseline demographic specification using data from Parts A-D together, but with CARA (a.k.a. "exponential") utility (Köbberling and Wakker 2005) (instead of CRRA, a.k.a. "power", utility). As in Online Appendix Section 6, we did not impose the assumption that the parameters for the coefficient of (absolute) risk aversion in the gain and in the loss domains (i.e., $\alpha_{\text {expo }}^{+}$and $\alpha_{\text {expo }}^{-}$, as well as the corresponding parameterized equations) are equal to one another in this specification with CARA utility. Lastly, we employed a two-step procedure in which we first estimated our baseline CPT model with the compromise effect separately for each participant, and then regressed each estimated parameter of interest on the demographic variables (and on the variables included in Other_variables). One limitation of this two-step analysis is that, to ensure that the MLE algorithm converged for sufficiently many participants, we had to reduce the number of parameters in the model by assuming that $\sigma_{q}$ is identical across all screens. ${ }^{1}$

We dropped from this analysis data from approximately three dozens of participants who had not provided their age, sex, and/or their highest level of education (unless they

\footnotetext{
${ }^{1}$ With this assumption, the MLE algorithm still failed to converge for 40 participants; we further dropped from the regression analysis in the second step 35 participants for whom the estimates of the parameter $\sigma_{q}$ were particularly large; this left 408 participants for the regression analysis, vs. 458 participants whose data were used in the other demographic specifications (as discussed below).
} 
indicated they were still currently studying). As in all the other analyses reported in the paper, we also dropped from this analysis data from the 28 participants for whom the MLE algorithm does not converge when the CPT model is estimated separately for each participant (in the model without the compromise effect, using data from Parts A-D together, and assuming that $\sigma_{q}$ is identical across all screens). This left 458 participants whose data were included in this analysis.

The dummy variables that control for race comprise a dummy variable that is equal to 1 if one's self-reported ethnicity is "Asian", as well as another dummy variable that is equal to 1 if one's self-reported ethnicity is "African-American", "Hispanic", "Native American", or "Other". Most participants for whom these dummies are both equal to 0 reported that their ethnicity is "Caucasian", but a few of these participants did not report their ethnicity.

The dummy variable indicating whether one has a college degree was defined based on responses to the question "what is the highest level of education you have completed?", with the five possible response categories "Additional education beyond college", "Completed college", "Some college", "Completed high school or GED", "Some high school". Participants who responded "Additional education beyond college" or "Completed college" were coded as having completed college. Only participants who were not fulltime students were asked this education question, so many observations are missing for our college dummy variable. Instead of dropping the corresponding participants from this analysis, we coded the college dummy as a constant ("-9") for these participants, and included in the parameterized equations for the parameters of interest another dummy variable indicating whether each participant has missing data for the college variable.

Many participants also had missing data for the SAT Math and the log parental income variables. We similarly coded these variables as constants for these participants and included, in the parameterized equations for the parameters of interest, dummy variables indicating whether each participant has missing data for these variables.

Only respondents who reported being full-time students were asked their parents' combined annual income. The log parental income variable was constructed from responses to the question "If you are a full-time student, what is your best guess of your parents' combined annual income?", with possible response categories "Under \$20,000", "Between \$20,000 and \$39,999", "Between \$40,000 and \$59,999", "Between \$60,000 and $\$ 79,999 "$, "Between $\$ 80,000$ and $\$ 99,999 "$, and "Over $\$ 100,000 "$. We replaced these responses with the midpoint of each interval (e.g., we coded parental income for participants who responded "Between $\$ 20,000$ and $\$ 39,999$ " as $\$ 30,000$ ); for the participants who responded "Under $\$ 20,000$ " and "Over $\$ 100,000$ ", we replaced these responses with " $\$ 15,000$ " and " $125,000 "$ ", respectively. Then, we took the logarithm of the resulting variable.

Online Appendix Table 9.1 shows summary statistics for these variables. 


\section{Online Appendix Table 9.1. Summary Statistics for the Demographic Covariates}

\begin{tabular}{lccccc}
\hline \hline & $N$ & Mean & SD & Min & Max \\
\hline \hline Age & 458 & 27.0 & 10.9 & 18 & 67 \\
Female & 458 & 0.62 & 0.49 & 0 & 1 \\
College & 190 & 0.65 & 0.48 & 0 & 1 \\
SAT Math & 328 & 670 & 111 & 200 & 800 \\
Parental income & 237 & 81,139 & 40,371 & 15,000 & 125,000 \\
Asian & 458 & 0.24 & 0.43 & 0 & 1 \\
Other race & 458 & 0.14 & 0.35 & 0 & 1 \\
\hline \hline
\end{tabular}

NOTE: Additional details on the variables can be found in the text. "Other race" is a dummy variable that is equal to 1 if one reported that one's ethnicity is "African-American", "Hispanic", "Native American", or "Other".

Online Appendix Table 9.2 reports the estimates of the parameters of interest in the baseline demographic specification.

Online Appendix Table 9.2. ML Estimates of Selected Parameters in the Baseline Demographic Specification

\begin{tabular}{|c|c|c|c|c|c|c|}
\hline & $\gamma$ & $\bar{\lambda}$ & $\alpha$ & $\underline{\beta}$ & $\pi_{1}$ & $\pi_{2}$ \\
\hline Age & $\begin{array}{c}0.0008 \\
(0.0009)\end{array}$ & $\begin{array}{l}-0.0052 \\
(0.0039)\end{array}$ & $\begin{array}{c}0.0027 \\
(0.0024)\end{array}$ & $\begin{array}{l}-0.0004 \\
(0.0027)\end{array}$ & $\begin{array}{c}0.0031^{* *} \\
(0.0013)\end{array}$ & $\begin{array}{l}-0.0002 \\
(0.0002)\end{array}$ \\
\hline Female & $\begin{array}{c}0.017 \\
(0.013)\end{array}$ & $\begin{array}{c}0.090 \\
(0.058)\end{array}$ & $\begin{array}{c}0.024 \\
(0.029)\end{array}$ & $\begin{array}{l}-0.016 \\
(0.031)\end{array}$ & $\begin{array}{c}0.023 \\
(0.026)\end{array}$ & $\begin{array}{l}-0.003 \\
(0.003)\end{array}$ \\
\hline College & $\begin{array}{c}0.000 \\
(0.023)\end{array}$ & $\begin{array}{l}-0.029 \\
(0.097)\end{array}$ & $\begin{array}{l}-0.062 \\
(0.054)\end{array}$ & $\begin{array}{c}0.030 \\
(0.067)\end{array}$ & $\begin{array}{c}-0.003 \\
(0.040)\end{array}$ & $\begin{array}{c}-0.003 \\
(0.004)\end{array}$ \\
\hline SAT Math & $\begin{array}{c}-0.00036^{* * *} \\
(0.00009)\end{array}$ & $\begin{array}{c}0.00127 * * * \\
(0.00029)\end{array}$ & $\begin{array}{c}0.00013 \\
(0.00021)\end{array}$ & $\begin{array}{c}0.00014 \\
(0.00026)\end{array}$ & $\begin{array}{c}-0.00025^{* *} \\
(0.00011)\end{array}$ & $\begin{array}{l}0.00003^{*} \\
(0.00002)\end{array}$ \\
\hline $\begin{array}{l}\log (\text { parental } \\
\text { income })\end{array}$ & $\begin{array}{l}-0.002 \\
(0.012)\end{array}$ & $\begin{array}{l}-0.038 \\
(0.064)\end{array}$ & $\begin{array}{c}0.031 \\
(0.028)\end{array}$ & $\begin{array}{c}0.013 \\
(0.037)\end{array}$ & $\begin{array}{l}-0.024 \\
(0.030)\end{array}$ & $\begin{array}{c}0.000 \\
(0.003)\end{array}$ \\
\hline $\begin{array}{l}\text { Log- } \\
\text { likelihood } \\
\text { Parameters } \\
\text { Individuals } \\
\text { Observations }\end{array}$ & $\begin{array}{c}-50,659 \\
79 \\
458 \\
28,396\end{array}$ & & & & & \\
\hline
\end{tabular}

NOTE: Standard errors are clustered by participant. The log-likelihood statistic is for the model without clustering. All estimates were obtained from one MLE. The estimates in each column indicate the effects of selected demographic covariates on the parameter at the top of the column.

$*$ significant at $10 \%$ level; ** significant at $5 \%$ level; *** significant at $1 \%$ level.

The estimates from the robustness specifications are available upon request. (We note that the MLE algorithm for the robustness specification that is identical to the baseline demographic specification but only uses data from Part A failed to converge; however, 
after 2,000 iterations, the estimates were consistent with those from the baseline and other robustness specifications.)

As mentioned in the main text, two results stand out across the baseline and robustness specifications. First, higher SAT Math scores are associated with lower $\gamma$-i.e., with lower risk aversion in the gain domain and higher risk aversion (or, equivalently, lower risk seeking) in the loss domain. Second, higher SAT Math scores are associated with higher loss aversion $(\lambda)$. As discussed in the main text, the first result is consistent with the existing literature, while the second is not. The parameter estimates in Online Appendix Table 9.2 imply that a 100-point increase in the SAT Math score is associated with a 0.036 -unit increase in the coefficient of relative risk aversion $(\gamma)$, and a 0.127 -unit increase in the loss aversion parameter $(\lambda)$; by comparison our estimates of $\gamma$ and $\lambda$ in our baseline CPT model with the compromise effect are 0.242 and 1.311 , respectively (from Table 1 of the main text).

While SAT scores are significantly associated with $\pi_{1}$ and marginally significantly associated with $\pi_{2}$, when considering the compromise effect parameters $c_{i}$ (where $i$ denotes the row of the alternative prospect on the screen, $i=1,2, \ldots, 7)$, these two effects cancel out. To see, recall that $c_{i}=\pi_{1}(i-4)+\pi_{2}\left(i^{2}-20\right)$. It follows that

$$
\frac{d c_{i}}{d S A T}=\frac{\partial c_{i}}{\partial \pi_{1}} \frac{\partial \pi_{1}}{\partial \mathrm{SAT}}+\frac{\partial c_{i}}{\partial \pi_{2}} \frac{\partial \pi_{2}}{\partial \mathrm{SAT}}=(i-4) \phi_{S A T}^{\pi_{1}}+\left(i^{2}-20\right) \phi_{S A T}^{\pi_{2}}
$$

Thus, in the first and last (i.e., seventh) rows, $\frac{d c_{1}}{d S A T}=-3 \phi_{S A T}^{\pi_{1}}-19 \phi_{S A T}^{\pi_{2}}$, and $\frac{d c_{2}}{d S A T}=$ $3 \phi_{S A T}^{\pi_{1}}+29 \phi_{S A T}^{\pi_{2}}$. Across both the baseline and the robustness specifications, $\phi_{S A T}^{\pi_{1}} \approx$ $10 \times \phi_{S A T}^{\pi_{2}}$, and so the effects of SAT Math scores on $\pi_{1}$ and $\pi_{2}$ effectively cancel out when considering the net effect of SAT Math scores on the compromise effect. 


\section{Estimates of $\gamma, \gamma^{+}, \gamma^{-}$, and $\lambda$ by EV Treatment in the Models with and Without the Compromise Effect}
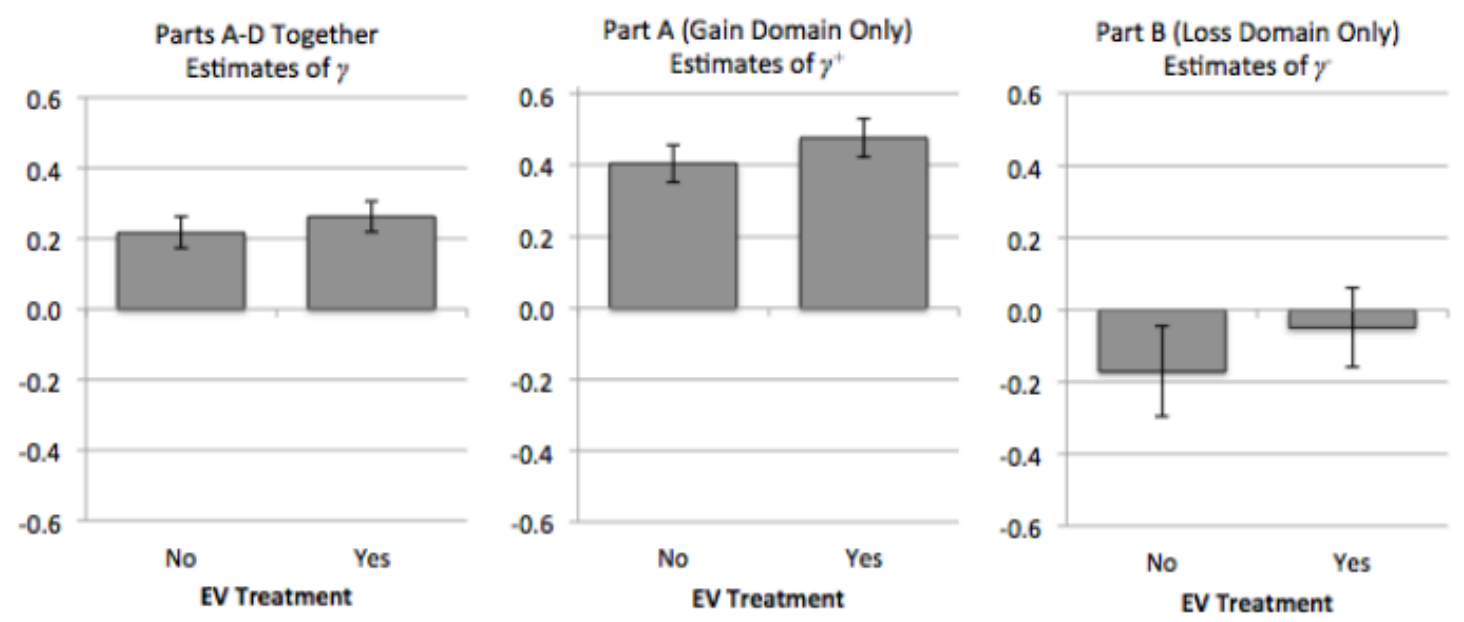

$\square$ Estimate $\mapsto 95 \%$ Confidence Interval

Online Appendix Figure 10.1. Estimates of $\gamma, \gamma^{+}$, and $\gamma^{-}$by EV treatment, from the model with the compromise effect. The negative estimates of $\gamma^{-}$for Part B reflect risk aversion in the loss domain, unlike what CPT predicts. ( $\gamma$ is not estimated for Parts C and D only because these parts have few questions.)

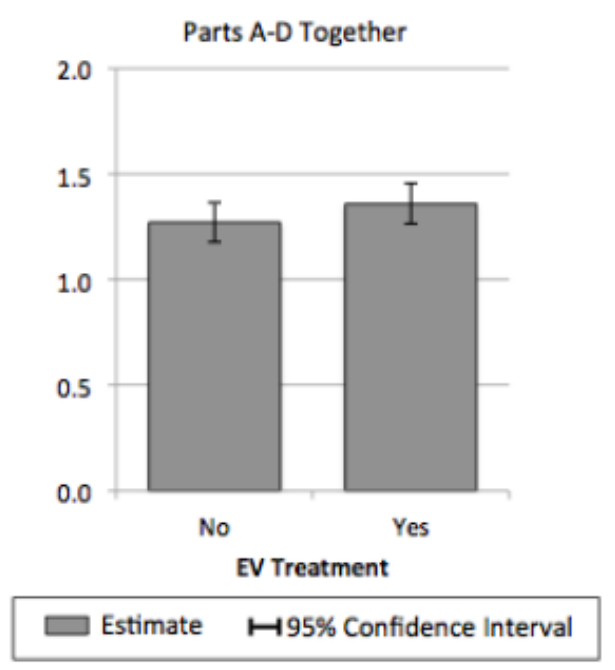

Online Appendix Figure 10.2. Estimates of $\lambda$ by EV treatment, from the model with the compromise effect, for Parts A-D together. ( $\lambda$ cannot be estimated for Part A only or Part B only because the questions in these parts are all in the gain or loss domains, and is not estimated for Parts C and D only because these parts have few questions.) 

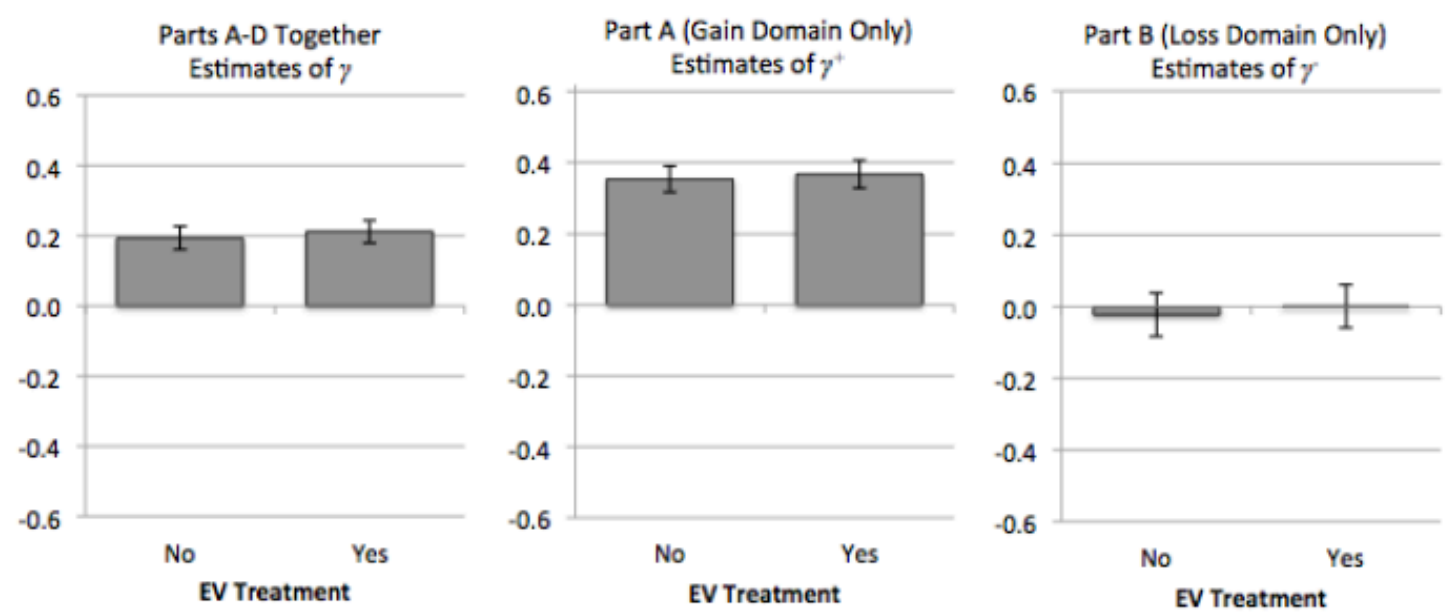

Estimate $\longmapsto 95 \%$ Confidence Interval

Online Appendix Figure 10.3. Estimates of $\gamma, \gamma^{+}$, and $\gamma^{-}$by EV treatment, from the model without the compromise effect. This figure is analogous to Online Appendix Figure 10.1, except that the estimated model does not control for the compromise effect.

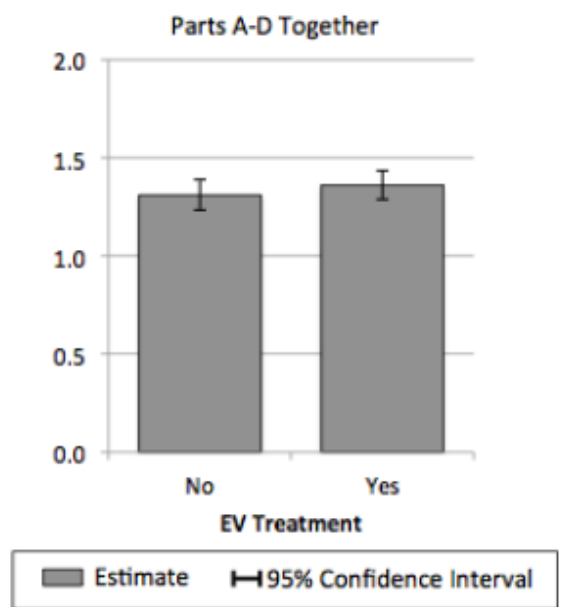

Online Appendix Figure 10.4. Estimates of $\lambda$ by EV treatment, from the model without the compromise effect, for Parts A-D together. This figure is analogous to Online Appendix Figure 10.2, except that the estimated model does not control for the compromise effect. 


\section{Stata Code to Estimate the Baseline CPT Model with the Compromise Effect}

We include below the Stata code used to estimate the baseline CPT model (with CRRA utility and the Prelec (1998) probability weighting function) with the compromise effect, using the data from Parts A-D and from all treatments together. The variable names in the code match the notation used in the main text.

Upon publication of the paper, we will post online the Stata code to estimate the other baseline CPT specifications that use the data from Parts A-D together (including specifications without the compromise effect, specifications that estimate the model for each treatment separately, and specifications with parameterized models). We will also post online the analogous specifications used for the three main sets of robustness checks which we describe in Section 3.4 of the main text. In addition, we will post online the deidentified choice data we collected in the experiment. (To ensure the privacy of the participants is not compromised, we will not post the data from the brief post-experiment questionnaire.)

Stata code:

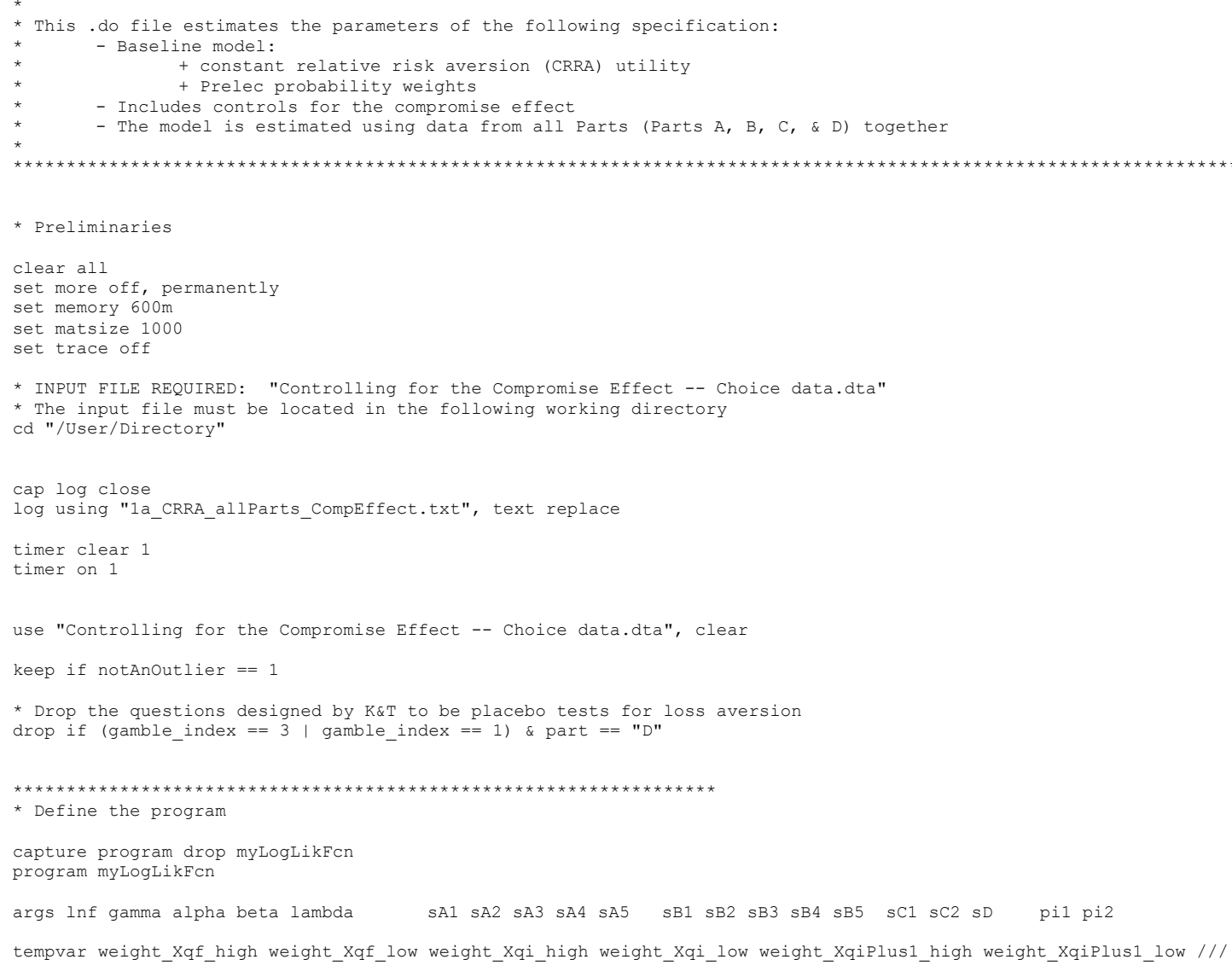


util_Xqf_high util_Xqf_low util_Xqi_high util_Xqi_low util_XqiPlus1_high util_XqiPlus1_low /// c_i c_iplus1 sigma ///

U Pqf U Pqi U PqiPlusi

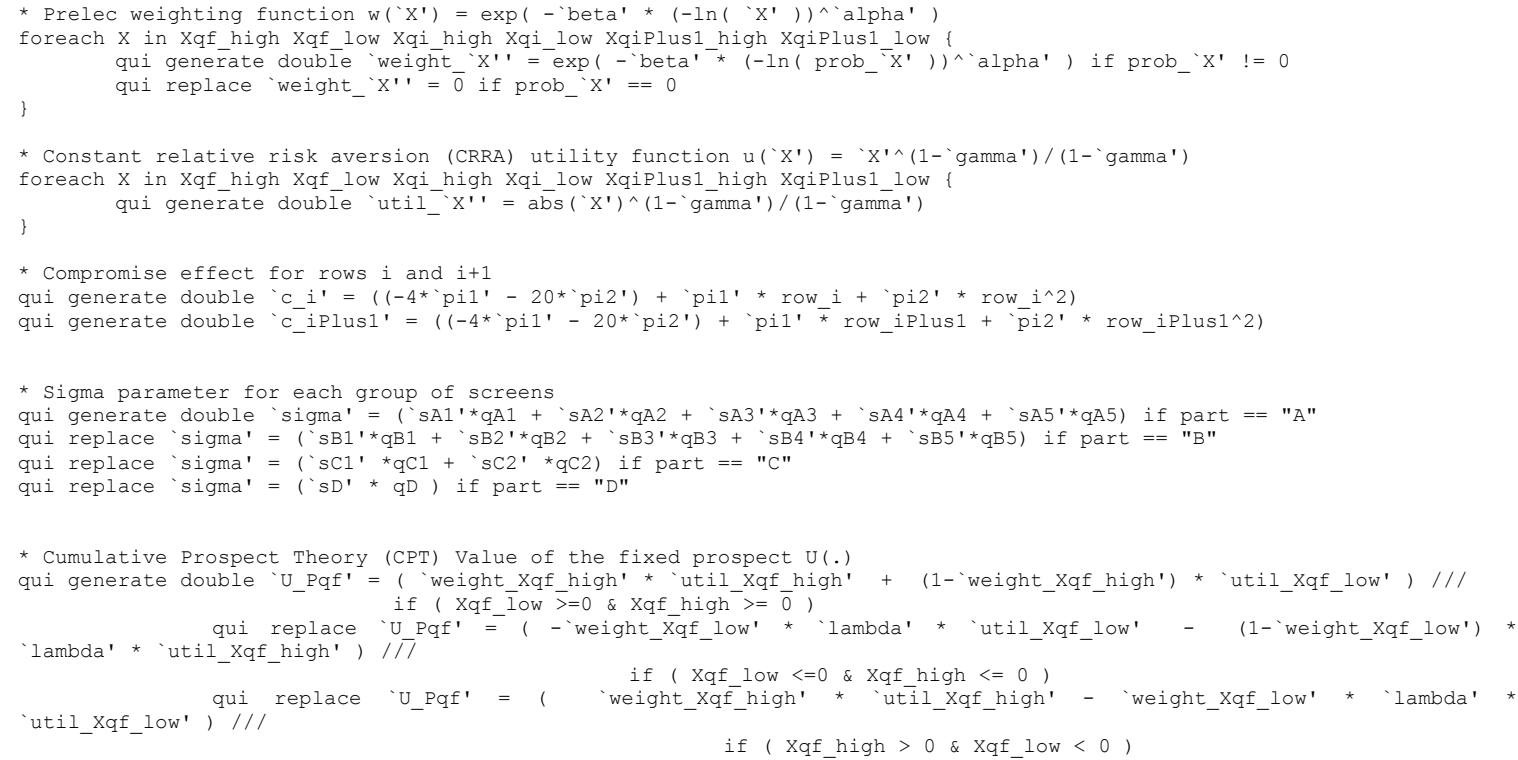

* The log likelihood function needs to be defined slightly differently when the alternative prospects are increasing from (a) to $(g)$.

* We subtract the compromise effect here because theoretically, c_iPlus1 > c_i when the alternative prospects are increasing.

quietly replace 'Inf' $=\ln (/ / /$

normal ( ('U_PqiPlus1' - 'U_Pqf' ) / 'sigma' - 'C_iPlus1' ) /// 


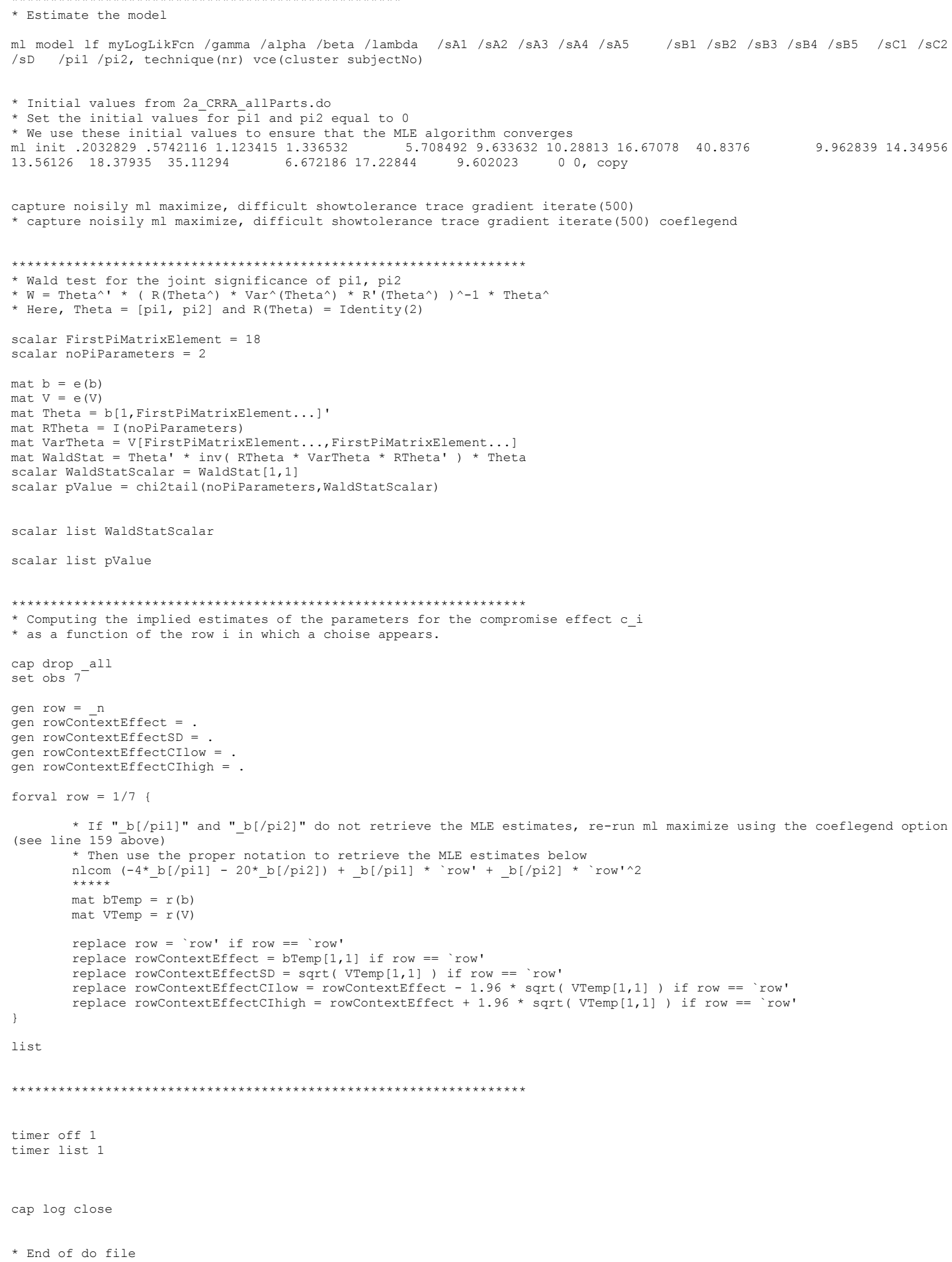




\section{Original Instructions of the Experiment}

\section{Informed Consent}

Please consider this information carefully before deciding whether to participate in this research.

\section{Purpose of the research:}

The purpose of this study is to examine individual decision-making in an experimental context.

\section{What you will do in this research:}

You will sit in front of a computer and be shown a series of questions regarding different monetary scenarios. Your task is simply to indicate which outcome you prefer. If you complete the study, you will have a chance to earn an additional payment (as described below under "Compensation").

\section{Time required:}

Participation will take approximately 30 to 45 minutes to complete.

\section{Risks:}

There are no anticipated risks associated with participating in this study. The effects of participating should be comparable to those you would experience from viewing a computer monitor for 30 to 45 minutes and using a mouse or keyboard.

\section{Benefits:}

At the end of the study, we will provide an explanation of the study and of our hypotheses. We will describe the potential implications of the results of the study both if our hypotheses are supported and if they are disconfirmed. If you wish, you can send an email message to Jonathan Beauchamp

(jpbeauch@fas.harvard.edu) or to Brendan Price (priceb@nber.org) and we will send you a copy of any manuscripts based on the research (or summaries of our results).

\section{Compensation:}

You will receive a participation fee of $\$ 15$ for completing the study. If you withdraw from the study without completing it, your participation fee will be decreased as follows:

- You will receive $\$ 15$ if you finish all four parts (A, B, C, and D).

- You will receive only $\$ 11$ if you finish only Parts A, B, and C.

- You will receive only $\$ 9$ if you finish only Parts A and B.

- You will receive only $\$ 7$ if you finish only Part A. 
- You will receive only $\$ 5$ if you finish none of the four parts of the study.

If you finish all four parts of the study, you will also have have a chance to earn an additional amount of money. At the end of the study, the computer will randomly choose a number from 1 to 6 . If it chooses a 6 (a one in six chance), one question from the first part of the study will be selected at random and you may receive an additional payment of no more than $\$ 400$ on the basis of your answer to that question. Depending on your choices, you may be paid in the form of a monetary gamble giving you a chance of gaining some amount of money and a chance of gaining no additional money.

Although some questions will concern possible monetary losses, you will not lose any money as a result of participating in this study.

If you do not finish all four parts, you will not have an opportunity to earn an additional amount of money.

You will be paid by check. In order to receive your payment, you must have listed your current mailing address on your CLER profile so we can mail you your check. Your check will be put in the mail no later than Friday, April 16.

\section{Confidentiality:}

Any information that is obtained in connection with this study and that can be identified with you will remain confidential. Your identity will not be stored with your data, and we will not collect your IP address. Your responses will be assigned a code number, and the list connecting your name with this number will be kept in a locked room and will be destroyed once all the data have been collected and analyzed. The data will be kept anonymously for future analysis.

\section{Participation and withdrawal:}

Your participation in this study is completely voluntary, and you may withdraw at any time by leaving the study website (no questions will be asked). If you choose to be in this study, you may subsequently withdraw from it at any time. If you withdraw during the course of the study, your participation fee will be determined as described above under "Compensation."

\section{Contact:}

If you have questions about this research, please contact Jonathan Beauchamp (jpbeauch@fas.harvard.edu) or Brendan Price (priceb@nber.org). You may also contact the faculty member supervising this work: David Laibson (dlaibson@harvard.edu).

\section{Whom to contact about your rights in this research, for questions, concerns, suggestions, or complaints that are not being addressed by the researcher, or research-related harm:}

Jane Calhoun, Harvard University Committee on the Use of Human Subjects in Research, 1414 Mass Ave., 2nd Floor, Cambridge, MA 02138. Phone: 617-495-5459. E-mail: jcalhoun@fas.harvard.edu

\section{Agreement:}

The nature and purpose of this research have been sufficiently explained and I agree to participate in this study. I understand that I am free to withdraw at any time without incurring any penalty. 


\section{Part A: Instructions}

In this part, you will make choices about 28 monetary scenarios. For example, a scenario might be:

A gamble gives you a 50\% chance of gaining $\$ 150$ and a $50 \%$ chance of gaining $\$ 50$ instead.

After each scenario is presented, you will be asked to indicate if you would prefer to take the gamble or to gain a fixed amount of money for sure. For example, you might be asked:

Would you rather...

Take the gamble OR Gain $\$ 80.50$

If this were an actual question, you would answer it by clicking on one of the two circles. You will be asked a series of such questions for each scenario. You will not be able to change your answers once you have submitted them.

At the end of the experiment, the computer will randomly choose a number from 1 to 6 . If it chooses 6 (a one in six chance), one question will be selected at random and you will be paid on the basis of your answer for that question. For example, if 6 were chosen and the above example question were picked, then depending on which circle you clicked, you would be paid either $\$ 80.50$ or the result of taking the gamble. (The result of taking the gamble would be determined randomly by the computer in accordance with the indicated percent chances.) We know some of the money amounts are large; however, if a large amount is selected to be paid, we will pay you that amount of money.

Because the computer might choose 6 , and because each of the following decision-making questions has a chance of being selected, you should answer each question as though that question determined your payment. It also helps us in our research if you answer all the questions as truthfully as you can.

There are no right or wrong answers here. Which choice you make is a matter of personal preference. Please pay careful attention to the amounts in each question and answer according to your own preferences. 


\section{Part B: Instructions}

In this part, you will again make choices about 28 monetary scenarios. For example, a scenario might be:

A gamble gives you a $75 \%$ chance of losing $\$ 40$ and a $25 \%$ chance of losing $\$ 20$ instead.

After each scenario is presented, you will be asked to indicate if you would prefer to take the gamble or to lose a fixed amount of money for sure. For example, you might be asked:

Would you rather...

Take the gamble OR Lose $\$ 30.70$

If this were an actual question, you would answer it by clicking on one of the two circles. You will be asked a series of such questions for each scenario. You will not be able to change your answers once you have submitted them.

The questions in this part are hypothetical only -- although they concern possible monetary losses, you will not be paid or have to pay any money to us for your answers in this part. Nonetheless, we ask you to answer all the questions as truthfully as you can, as if they were associated with real monetary outcomes.

There are no right and wrong answers here. Which choice you make is a matter of personal preference. Please pay careful attention to the amounts in each question and answer according to your own preferences. 


\section{Part C: Instructions}

In this part, you will make choices about four monetary scenarios. Each scenario will describe a gamble giving you a 50\% chance of losing some amount of money and a 50\% chance of instead gaining some amount of money that changes from question to question. For example, a scenario might begin:

A gamble gives you a $50 \%$ chance of losing $\$ 80$ and ...

A question might then complete the scenario with:

... a $50 \%$ chance of gaining $\$ 120.50$ instead.

For each question, you will be asked to indicate whether you would prefer to take the gamble or not to take the gamble:

Would you rather ...

Take the gamble OR Not take the gamble

If this were an actual question, you would answer it by clicking on one of the two circles. You will be asked a series of such questions for each scenario. You will not be able to change your answers once you have submitted them.

The questions in this task are hypothetical only -- although they concern possible monetary losses, you will not be paid or have to pay any money to us for your answers in this part. Nonetheless, we ask you to answer all the questions as truthfully as you can, as if they were associated with real monetary outcomes.

There are no right and wrong answers here. Which choice you make is a matter of personal preference. Please pay careful attention to the amounts in each question and answer according to your own preferences. 


\section{Part D: Instructions}

In this part, you will make choices about four monetary scenarios. Within each scenario, "gamble 1" will stay the same but "gamble 2" will change from question to question. For example, the scenario might begin:

Gamble 1 gives you a 50\% chance of losing \$60 and a 50\% chance of gaining \$200 instead.

A description of Gamble 2 might begin:

Gamble 2 gives you a 50\% chance of losing $\$ 100$ and ...

A question might then complete the description of Gamble 2 with:

... a $50 \%$ chance of gaining $\$ 300.10$ instead.

For each question, you will be asked to indicate whether you would prefer to take Gamble 1 or to take Gamble 2, as completed by the question:

Would you rather ...

Take gamble 1 OR $\bigcirc$ Take gamble 2

If this were an actual question, you would answer it by clicking on one of the two circles. You will be asked a series of such questions for each scenario. You will not be able to change your answers once you have submitted them.

The questions in this task are hypothetical only -- you will not be paid or have to pay any money to us for your answers. Nonetheless, we ask you to answer all the questions as truthfully as you can, as if they were associated with real monetary outcomes.

There are no right and wrong answers here. Which choice you make is a matter of personal preference. Please pay careful attention to the amounts in each question and answer according to your own preferences. 


\section{Debrief}

This experiment was conducted to explore people's attitudes towards gains and losses. Prior research suggests that people dislike financial risks and are more sensitive to potential losses than to potential gains. Economic theory provides methods of measuring risk and loss attitudes on the basis of choices about monetary gambles. However, decisions in laboratory experiments are often influenced by seemingly irrelevant factors. A main purpose of our study is to determine whether people's willingness to take monetary gambles is affected by the wording and ordering of the questions we ask.

This concludes your participation in our study. Thank you for participating! We will mail your payment to the mailing address listed on your CLER profile. We will put your check in the mail no later than Friday, April 16. Please contact the study administrator at laibson.study@gmail.com if you have any questions. 


\section{References}

See the main text's References section (all works cited in this Online Appendix are also cited in the main text). 